Portland State University

PDXScholar

1990

\title{
Optimization and Thermodynamic Performance Measures of a Class of Finite Time Thermodynamic Cycles
}

Joseph D. Walters

Portland State University

Follow this and additional works at: https://pdxscholar.library.pdx.edu/open_access_etds

Part of the Physics Commons

Let us know how access to this document benefits you.

Recommended Citation

Walters, Joseph D., "Optimization and Thermodynamic Performance Measures of a Class of Finite Time Thermodynamic Cycles" (1990). Dissertations and Theses. Paper 1186.

https://doi.org/10.15760/etd.1185

This Dissertation is brought to you for free and open access. It has been accepted for inclusion in Dissertations and Theses by an authorized administrator of PDXScholar. Please contact us if we can make this document more accessible: pdxscholar@pdx.edu. 
OPTIMIZATION AND THERMODYNAMIC PERFORMANCE MEASURES

FOR A CLASS OF FINITE TIME THERMODYNAMIC CYCLES

by

JOSEPH D. WAITERS

A dissertation submitted in partial fulfillment of the requirements for the degree of

DOCTOR OF PHILOSOPHY

in

ENVIRONMENTAI SCIENCES AND RESOURCES:

PHYSICS

Portland State University

(C) 1990 
TO THE OFFICE OF GRADUATE STUDIES:

The members of the committee approve the dissertation of Joseph D. Walters presented August 1, 1990.

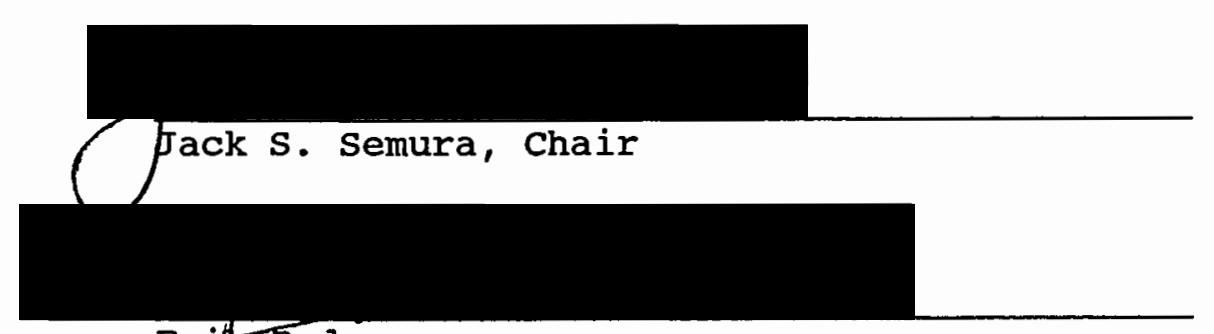

Erit Bodegom

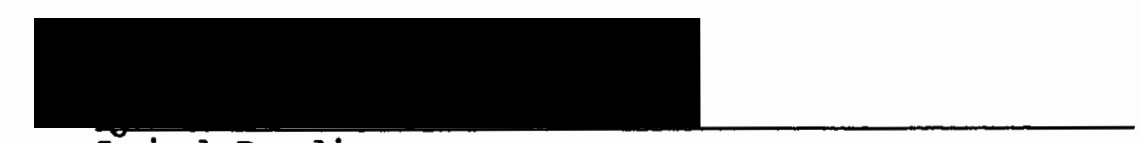

Laird Brodie

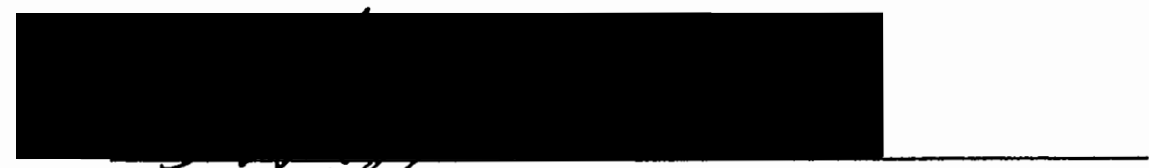

stänlex Hilłman

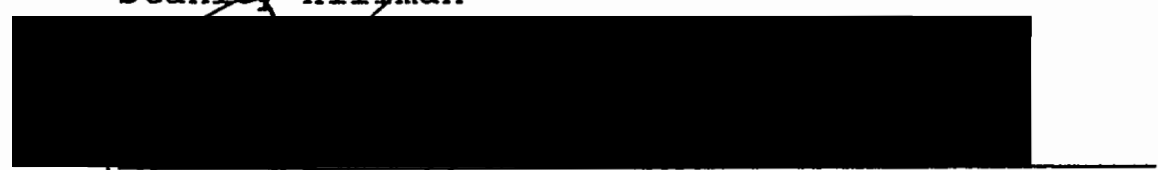

Ansel Johnson

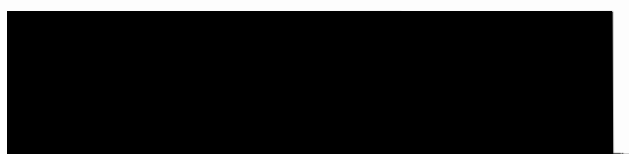

Gavin Bjork (

APPROVED:

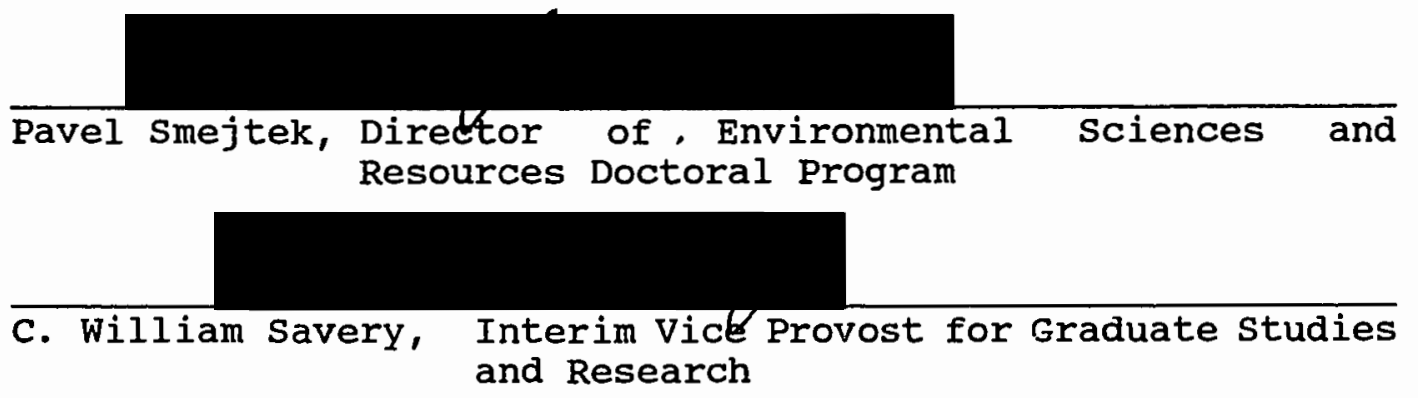




\section{DEDICATION}

This dissertation is dedicated to the memory of my mother Doris Walters and to my wife Sharron Walters. Doris, throughout her loosing battle with cancer, revealed to me the meaning of personal courage, dedication, and strength. During my years of graduate school, Sharron has endured much more than any companion should ever have to in support of an at times crazy person. Luckily for me, Sharron and I are still on speaking terms. 


\section{ACKNOWLEDGEMENTS}

I would like to acknowledge the following people for their support in bringing this project to fruition: My dissertation advisor Jack Semura for his patience and guidance, Eric Bodegom and Laird Brodie for helpful discussions and insights, and Joel Nissen, Ed Buck, and Collin wright for their friendship. 
TABLE OF CONTENTS

PAGE

ACKNOWLEDGEMENTS . . . . . . . . . . . . . . . iii

LIST OF TABLES . . . . . . . . • . . . . . . . . . . vi

LIST OF FIGURES . . . . . . . . . . . . . . . . . . vii

CHAPTER

I INTRODUCTION . . . . . . . . . . . . 1

Heat Engines . . . . . . . . . . 1

Refrigeration Cycles . . . . . . . 3

II MAXIMUM EFFICIENCY AT FINITE COOLING POWER

OF A FINITE TIME CARNOT REFRIGERATION CYCLE. . 13

Introduction . . . . . . . . . 13

Refrigeration Cycle Model . . . . . . 16

Maximum Efficiency . . . . . . . . 22

Efficiency at Maximum

cooling Power

Summary and Conclusion . . . . . . . 32

III OPTIMAL OPERATING MODES OF A FINITE TIME

REFRIGERATION CYCLE . . . . . . . . . 34

Introduction . . . . . . . . . . 34

Refrigeration Cycle Model . . . . . . 37

optimization of Cycle Branches . . . . 41

objective Functions

Optimizing Heat Transfer 
Optimal FTCRC Operating Modes . . . . 50

Maximum Efficiency

Maximum Cooling Power

Minimum Power Consumption

Minimum Irreversible Entropy

Summary and Conclusion . . . . . . 69

IV MAXIMUM EFFICIENCY OF A FINITE TIME

REFRIGERATION CYCLE WITH NON-IDEAL HEAT

SWITCHES . . . . . . . . . . . . . 74

Introduction ............ 74

Refrigeration Cycle Model . . . . . 76

Optimization of Cycle Branches . . . . 83

objective Function

optimizing Heat Transfer

Maximum Efficiency . . . . . . . 91

Summary and Conclusion . . . . . . 104

REFERENCES ................ 106

APPENDICES

A CONVEXITY/CONCAVITY OF OBJECTIVE FUNCTIONS • . 108

B EQUIVALENCE OF OPTIMIZING THE NET HEAT

TRANSFER INTO THE WORKING FLUID AND THE NET

HEAT TRANSFER INTO THE EXTERNAL HEAT

RESERVOIRS . . . . . . . . . . . . . . 115 
LIST OF TABLES

TABLE

PAGE

I Comparison of the Quasistatic Carnot

COP with the COP at Finite cooling

Power of a Finite Time carnot

Refrigeration Cycle. . . . . . . . 31

II Comparison of the Quasistatic Carnot

COP with the COP at Finite cooling

Power of an FTCRC with Non-Ideal Heat

Switches . . . . . . . . . 102 


\section{LIST OF FIGURES}

FIGURE

PAGE

1. Temperature-Entropy State Space Diagram of a Finite Time Carnot Refrigeration Cycle . 17

2. Curve a: Reduced Coefficient of Performance, $\eta$, and Curve b: Reduced cooling Power, $\tilde{P}_{c}$, as Functions of the Reduced cycling Frequency $f$. . . . . . . . . 27

3. Quasistatic Carnot coefficient of Performance, $\epsilon_{\circ}$, and Finite Time Coefficient of Performance at Maximum Cooling Power, $\epsilon_{m}$, vs $T_{H} / T_{L}$ Thermal

Reservoir Temperature Ratio . . . . . 29

4. Temperature-Entropy state Space Diagram of a Finite Time Refrigeration Cycle . . . 38

5. Three Dimensional Efficiency Space . . . . 54

6. Reduced Coefficients of Performance, $\eta$, vs Refrigeration Cycling Frequency, $\vec{f}$, for Four Optimal Operating Modes . . . 58

7. Reduced Coefficients of Performance, $\eta$, vs Reduced cooling Power, $\tilde{P}_{c}$, for Three Optimal Refrigeration Cycling Modes . . 66 
8. Three Dimensional Surface of Reduced Cycling Frequency, $\tilde{f}$, cooling Power, $\tilde{P}_{c}$, and Coefficient of Performance, $\eta$. . . 68

9. Temperature-Entropy Space Diagram of a Finite

Time Refrigeration Cycle with Non-Ideal

Heat Switches . . . . . . . . . 77

10. Schematic of a Finite Time Refrigeration

Cycle with Non-Ideal Heat Switches . . 78

11. Three Dimensional Efficiency Space for an FTCRC with Non-Ideal Heat Switches . . 93

12. Reduced Coefficient of Performance, $\eta$, vs Reduced Cycling Frequency, $f$. . . 98

13. Reduced Coefficient of Performance, $\eta$, vs Reduced Cooling Power $\tilde{P}_{m}$. . . . . . 101 
AN ABSTRACT OF THE DISSERTATION OF Joseph D. Walters for the Doctor of Philosophy in Environmental Sciences and Resources: Physics presented August 1, 1990.

Title: Optimization and Thermodynamic Performance Measures of a class of Finite Time Thermodynamic Cycles.

APPROVED BY THE MEMBERS OF THE DISSERTATION COMMITTEE:
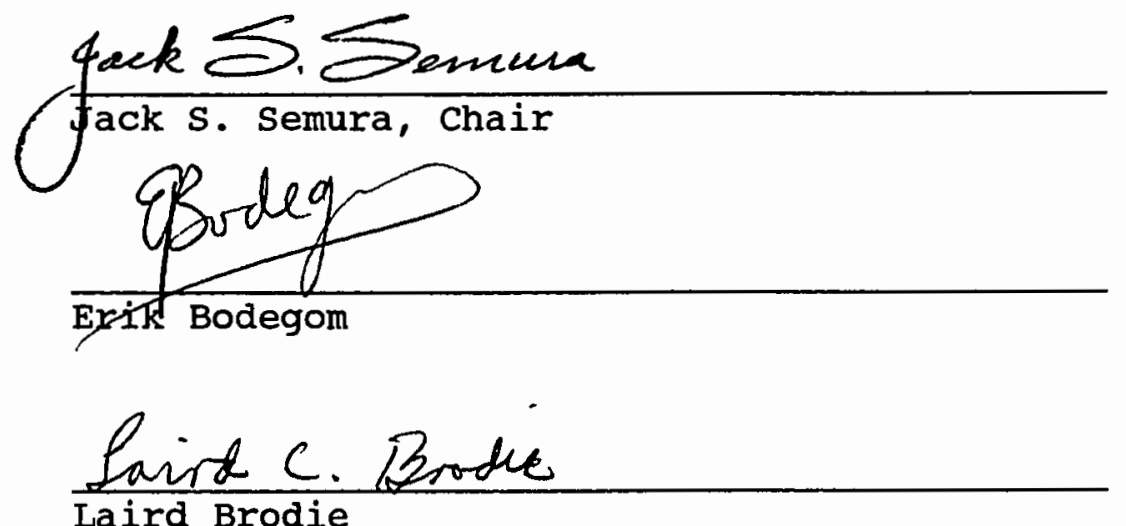
Laird Brodie
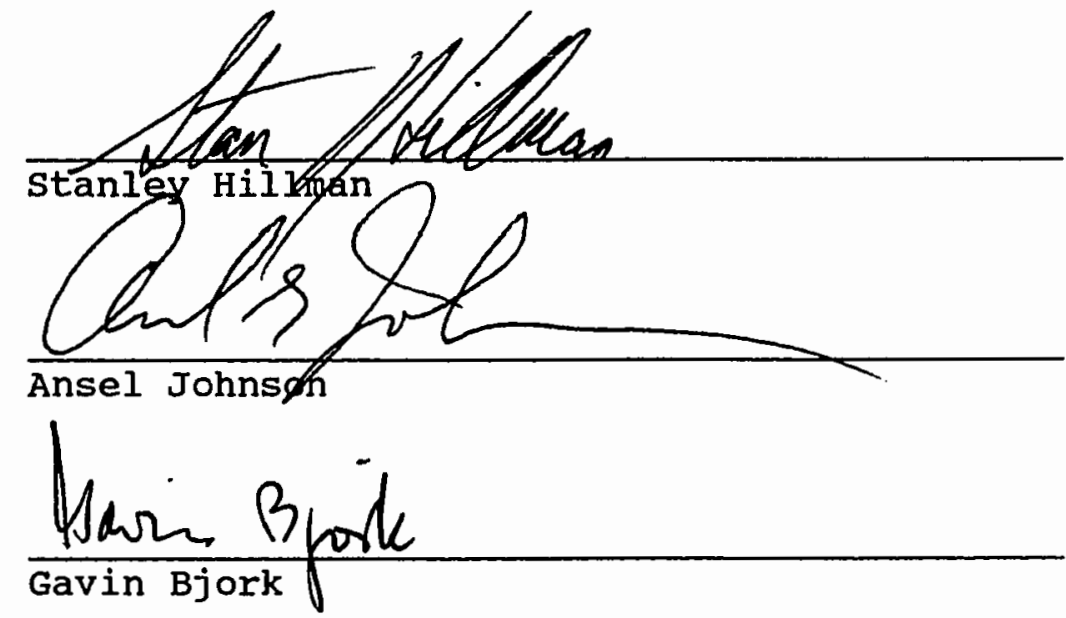

Traditionally, the thermal efficiency of energy conversion processes are compared to the quasistatic Carnot 
bounds on performance. Despite the general theoretical importance of the limiting carrot efficiencies, the idealized nature of the quasistatic Carnot refrigeration cycle, (for example, in which no cooling power is produced) limits the usefulness of the carnot bound as a limiting thermal efficiency estimate for refrigeration cycles operating at nonzero cycling frequencies and producing cooling power.

In this study, modifications to the quasistatic Carnot cycle are developed in order to formulate improved theoretical bounds on the thermal efficiency of certain refrigeration cycles that produce finite cooling power. The modified refrigeration cycle model is based on the idealized endoreversible finite time cycle. Two of the four cycle branches are defined as reversible adiabats, and the other two are the high and low temperature branches along which finite heat fluxes couple the refrigeration cycle with external heat reservoirs.

This finite time model has been used to obtain the following results: First, the performance of a finite time Carnot refrigeration cycle (FTCRC) is examined. In the special symmetric case of equal heat transfer coefficients along the high and low temperature branches, it is found that by optimizing the FTCRC to maximize thermal efficiency and then evaluating the efficiency at peak cooling power, a new bound on the thermal efficiency at finite cooling power 
of certain Carnot refrigeration cycles is given by

$$
\boldsymbol{\epsilon}_{m}=\left(\tilde{\tau}_{m}^{2}\left(\frac{T_{H}}{T_{L}}\right)-1\right)^{-1},
$$

where $T_{H}$ and $T_{L}$ are the absolute high and low temperatures of the two heat reservoirs, respectively, and $\tilde{\tau}_{m}=\sqrt{2}+1 \times 2.41$ is the dimensionless cycle period at maximum cooling power.

second, a finite time refrigeration cycle (FTRC) is optimized to obtain four distinct optimal cycling modes that maximize efficiency and cooling power, and minimize power consumption and irreversible entropy production. An efficiency space is defined, and the global maximum efficiency trajectory is obtained within this space that defines the maximum efficiency mode of operation. Efficiency trajectories defining the latter three optimal operating modes are also obtained. It is found that all four respective trajectories converge to the global quasistatic Carnot maximum efficiency in the zero cycling frequency limit. By expanding the efficiencies of each optimal operating mode to first order in cycling frequency and in the special symmetric case, the maximum efficiency and minimum irreversible entropy production modes are determined to be equally efficient. In addition, simple analytic expressions are obtained for the coefficients of performance at maximum cooling power within each of the four 
optimal operating modes. These latter efficiencies depend only on the temperature ratio of the two external isothermal heat reservoirs. Under certain limiting conditions the bounding efficiency at maximum cooling power shown above is obtained.

Third, the problem of imperfect heat switches linking the working fluid of an FTRC to external heat reservoirs is studied. Non-ideal heat switches significantly affect the efficiency characteristics of endoreversible FTRC's. The efficiency space representing the performance of an FTRC with non-ideal heat switches is severely folded relative to the efficiency surface representing an FTCRC with ideal heat switches. The degree to which the efficiency surface for the non-ideal case is folded and contains negative efficiencies is indicative of heat-leakage through non-ideal switches. By analytically and numerically optimizing the FTRC, the cycling mode corresponding to operation at maximum efficiency is obtained. It is found that there exist two distinct optimum cycling conditions for a refrigeration cycle with heat leaks due to non-ideal heat switches: 1) operation at the global maximum in efficiency, and 2) operation at the frequency of maximum cooling power. The efficiency evaluated at maximum cooling power, and the global maximum efficiency may provide improved bench-mark bounds on the efficiencies of certain real irreversible refrigeration cycles. 
CHAPTER I

\title{
INTRODUCTION
}

\begin{abstract}
More efficient energy conversion systems are of increasing importance as energy resources for currently used systems dwindle and demand for these shrinking resources continues to grow. Between 1971 and 2001, the U.S. demand for energy has been projected to double (1). This is significant since energy consumption in the U.S. accounts for approximately a third of the annual worldwide energy consumed. In addition, the annual worldwide demand for energy during the same time period is expected to triple. Therefore, it is important to attempt to obtain a better understanding of the thermodynamics of energy conversion processes and thermal efficiency.
\end{abstract}

\section{HEAT ENGINES}

Traditionally, the thermal efficiency of energy conversion processes are compared to the quasistatic Carnot bounds on performance. For a quasistatic heat engine operating cyclically between two isothermal heat reservoirs, the Carnot bound states that thermal energy cannot be converted to work more efficiently than the theoretical maximum 


$$
\eta=1-\frac{T_{H}}{T_{L}}
$$

where $T_{H}$ and $T_{L}$ are the temperatures of the hot and cold heat reservoirs. Despite the general theoretical importance of this limiting carnot efficiency, the idealized nature of the quasistatic carnot heat engine in which no power is produced limits the usefulness of Eq. (1.1) as an estimate of the limiting thermal efficiency of heat engines cycling at nonzero frequencies and producing power.

In recent years increasing interest has been focused on the problem of formulating improved thermodynamic bounds that better reflect the performance limits of heat engines. Much of this interest has been focused on the description and optimization of the properties of heat engines operating with finite cycling times. In particular, the works of Curzon and Ahlborn (2), Salamon (3,4), Rubin (5), Andresen (6-8), and others (9-11) have incorporated certain time dependent properties of real engines into standard quasistatic heat engine models. By including time dependence, certain inherently irreversible features that are absent from the quasistatic thermodynamic description become a natural part of these finite time models. Examples of important questions that can be examined include how to optimally configure heat engines either for maximum power production or for maximum efficiency at finite power. 
The thermal efficiency of a heat engine operating at maximum power, first obtained by Curzon and Ahlborn (2), appears to be useful enough as a thermodynamical bound on the efficiencies of heat engines as to be worth stating here. For a class of heat engines operating in finite time (i.e. nonzero cycling frequencies) with linear Newton's law heat transfer and with equal heat transfer coefficients at the high and low temperatures, the efficiency at maximum power is found to be

$$
\eta_{C A}=1-\left(\frac{T_{H}}{T_{L}}\right)^{1 / 2},
$$

where $T_{L} / T_{H}$ is the ratio of the low and high temperatures of the heat reservoirs. It is worth noting that the curzonAhlborn efficiency of Eq. (1.2) depends only on the temperature ratio of the heat reservoirs and not on other cycle specific details such as volume or pressure constraints. It is this similarity to the quasistatic Carnot efficiency that makes $\eta_{C A}$ such an intriguing result. This is the reason why the carnot bound on heat engine thermal efficiency is so generally used.

\section{REFRIGERATION CYCLES}

In the case of the quasistatic Carnot refrigeration cycle, heat cannot be transported from a low temperature heat reservoir to a reservoir at a higher temperature more 
effectively than the theoretical maximum coefficient of performance (COP) given by

$$
\epsilon_{0}=\left(\frac{T_{H}}{T_{L}}-1\right)^{-1} \text {. }
$$

In a way analogous to the previously discussed efficiency of the quasistatic Carnot heat engine, the COP of a quasistatic Carnot refrigeration cycle producing no cooling power, as given by Eq. (1.3), is usually much too optimistic for refrigeration cycles that operate in finite time and which do produce finite cooling power.

In this dissertation, I develop modifications of the reversible carnot cycle in order to formulate improved theoretical bounds on the thermal efficiency of certain refrigeration cycles while attempting to retain much of the inherent simplicity which made the original quasistatic Carnot estimate so generally useful. Refrigeration cycle models of the types described in chapters II, III, and IV with Newton's law of cooling governing heat transfer constitute a set of simple refrigeration cycle models that can be used to study this problem. The cycle model is based on the idealized endoreversible cycle (5) in which two of the four cycle branches are defined to be reversible adiabats, and the other two are the high and low temperature isothermal branches along which finite heat fluxes couple the refrigeration cycle with external heat reservoirs. 
of primary interest in fulfilling the goals of this dissertation is obtaining optimal refrigeration cycle operating modes (i.e. different ways of cycling the refrigerator) for several variations of the basic refrigeration cycle model. Optimal operating modes that extremize such cycle properties as thermal efficiency, cooling power, power production, and irreversible entropy production could be obtained by using analytic and numerical optimization techniques. Optimizations of this type yield the detailed time paths followed to achieve optimal performance. Further, by examining certain maxima and minima within different optimal refrigeration cycle operating modes, new bounds on certain properties of refrigeration cycles operating in finite time may be obtained.

Towards these goals, the classical variational calculus, optimization theory, and numerical computational methods have been used with much success and have provided many interesting and important results in studies of finite time heat engines $(3-11)$. A typical optimization defining an optimal cycling mode proceeds as follows:

1) The amount of heat flowing in and out of a refrigeration cycle's working fluid over a complete cycle determines the performance characteristics of the refrigeration cycle. For the class of problems to be examined, refrigeration cycle performance will be gauged by 
the degree to which a particular finite time refrigeration cycle operating mode extremizes specific objective functions such as efficiency or cooling power. Therefore, it is important to know how maximizing or minimizing the heat flowing through the working fluid affects these and other objective functions. By taking the derivatives of objective functions with respect to the heats absorbed and rejected from the working fluid and examining the signs of these derivatives, it should be possible to determine if refrigeration cycle performance is optimized by maximizing or minimizing heat transfer.

2) The temperature of the working fluid as a function of time along the high and low temperature cycle branches determines the amount of heat transferred to and from the working fluid of the FTRC. Classical variational methods, such as the Euler-Lagrange method, could be used to obtain the temperature of the working fluid as a function of time that optimizes heat transfer between the working fluid and the external reservoirs.

3) Finally, the relative distribution of cycling time devoted to heat transfer along the high and low temperature cycle branches is to be optimized. This approach has been useful in many studies of finite time heat engines $(2,3,5,9,11)$. The choice of the appropriate optimization method to use is determined by the complexity of the constituent equations in the optimization. Examples of 
optimization methods are given below in the order they might be used for simple to increasingly complicated optimizations. These methods are the standard EulerLagrange variational method, the Kuhn-Tucker optimization method, and numerical computational methods. Euler-Lagrange and Kuhn-Tucker methods could be used for optimizations that can be performed analytically, and numerical computational methods could be used for problems that are difficult to solve analytically.

Optimizations following the general form described above are presented in chapter II, III, and IV, and yield FTRC operating modes that extremize certain objective functions such as efficiency and cooling power producing new bounds on the performance of certain carnot like refrigeration cycles operating in finite time.

In chapter II, the performance of a finite time carnot refrigeration cycle (FTCRC) operating endoreversibly at finite cycling times with irreversible heat transfer coupling the working fluid to two external isothermal heat reservoirs is examined. In particular, the optimal refrigeration cycle operating mode that maximizes thermal efficiency is studied. In the special symmetric case of equal heat transfer coefficients along the high and low temperature branches, it is found that by optimizing the FTCRC model to maximize thermal efficiency and then evaluating the efficiency at peak cooling power, a new bound 
on the thermal efficiency at finite cooling power of Carnot refrigeration cycles is given by

$$
\epsilon_{m}=\left(\tilde{\tau}_{m}^{2}\left(\frac{T_{H}}{T_{L}}\right)-1\right)^{-1},
$$

where $T_{H}$ and $T_{L}$ are the absolute high and low temperatures of the two heat reservoirs, respectively, and $\tilde{\tau}_{m}=\sqrt{2}+1 \propto 2.41$ is the dimensionless cycle period at maximum cooling power. It is proposed that this simple and new result for $\epsilon_{m}$ may be more useful than the quasistatic Carnot COP as a theoretical bound on thermal efficiency for certain Carnot refrigeration cycles that maximize thermal efficiency and produce cooling power.

In Chapter III, efficiency and cooling power differences are examined and compared between the four different refrigeration cycling modes that extremize thermal efficiency, cooling power, power consumption, and irreversible entropy production. An endoreversible finite time refrigeration cycle (FTRC) model is defined and subsequently is operated at finite cycling times with irreversible heat transfer coupling the working fluid to two isothermal external thermal reservoirs. Whereas in chapter II the finite time carnot refrigeration cycle is defined such that heat transfer occurs isothermally, heat transfer is not assumed to be isothermal in the FTRC studied in 
Chapter III. Using classical Euler-Lagrange variational methods (12), it is found that regardless of the cycling mode being considered, optimal operating modes are attained when heat transfer occurs isothermally and when the adiabatic branches are traversed instantaneously. Using this optimum cycle configuration and employing the KuhnTucker optimization conditions (13), the FTRC is further optimized to obtain the four distinct cycling modes that maximize thermal efficiency and cooling power, and minimize power consumption and irreversible entropy production. An efficiency space is defined and the global maximum efficiency trajectory is obtained within this space that defines the maximum efficiency mode of operation. Efficiency trajectories defining the latter three optimal operating modes are also obtained, and it is found that all four respective trajectories converge to the global quasistatic Carnot maximum efficiency in the zero cycling frequency limit. Expanding the efficiencies of each optimal operating mode to first order in cycling frequency and in the special symmetric case of equal heat transfer coefficients along the high and low temperature heat transfer branches, the maximum efficiency and minimum irreversible entropy production modes are determined to be equally efficient. Finally, simple analytic expressions are obtained for the coefficients of performance at maximum cooling power within each of the four optimal operating 
modes which depend only on the temperature ratio of the two external isothermal heat reservoirs. Under certain limiting conditions the bounding efficiency $\epsilon_{m}$ at maximum cooling power obtained in Chapter II is obtained in Chapter III. In Chapter IV, the problem of imperfect heat switches linking the working fluid of a refrigeration cycle to the external heat reservoirs is studied. If these switches do not function ideally, a significant source of irreversibility in the form of heat leaks may exist. For example, if the refrigeration cycle is Carnot like then when traversing the low temperature isothermal branch, heat is absorbed in the usual way from the low temperature reservoir into the working fluid, but due to imperfect thermal switching, heat will also leak into the working fluid from the high temperature reservoir. In a similar way, heat will leak back into the low temperature reservoir while operating the refrigeration cycle along the high temperature isothermal branch. Problems of this type can be especially prevalent in magnetic refrigeration systems $(14,15)$. It is important, therefore, to understand the effects of imperfect switching on cycle efficiency. An extended finite time refrigeration cycle model incorporating imperfect heat switches is used to study this problem.

As in Chapter III, Euler-Lagrange variational methods are used to show that optimum heat transfer occurs isothermally even with imperfect heat switches. However, 
because this problem is sufficiently more complicated mathematically than those discussed in Chapters II and III, numerical optimization methods have been used to obtain the optimum cycling mode corresponding to operation at maximum efficiency.

The performance of finite time carnot refrigeration cycles with perfect and imperfect heat switches are compared. Relative to a FTCRC with perfect switches, imperfect heat switches significantly alter the thermal efficiency and cooling power characteristics of finite time endoreversible refrigeration cycles. Most notably, an FTCRC with imperfect switches has certain operating regimes in which cooling power and efficiency are negative. In addition, it is found that there exist two distinct optimum cycling conditions for a cycle with imperfect heat switches: operation at the global maximum in efficiency, and operation at the frequency of maximum cooling power. The coefficient of performance evaluated at maximum cooling power, and the global maximum of the coefficient of performance may provide improved bench-mark bounds for the efficiencies of real irreversible refrigeration cycles.

It is hoped that the generalized finite time thermodynamics of refrigeration cycles may enrich our fundamental knowledge of thermodynamics, and yield insights helping us develop more efficient energy conversion methods for the future so that a suitable balance between energy 
consumption and energy resource availability can be attained. 
CHAPTER II

MAXIMUM EFFICIENCY AT FINITE COOLING POWER OF A FINITE TIME CARNOT REFRIGERATION CYCLE

\section{INTRODUCTION}

Traditionally, the efficiencies of thermal energy conversion systems are compared with the quasistatic carnot bounds on performance first obtained in the early 19th century and which have become a part of every physicist's education. As a practical matter, it is not generally worthwhile to operate cycles in the quasistatic extreme, either in the case of the engine or the refrigeration cycle, since cycles operating quasistatically work infinitely slowly and consequently produce vanishing power. Thus, we are faced with the paradoxical situation in which the maximum thermodynamic efficiency can only be attained in the limit in which useful power cannot be extracted. In fact, real heat engines and refrigeration cycles operate nonquasistatically at finite cycling times while irreversibly producing power and cooling power, respectively.

In this chapter, modifications of the reversible carnot cycle will be developed in order to formulate an improved theoretical bound on the thermal efficiency of refrigeration 
cycles while attempting to retain much of the inherent simplicity which made the original quasistatic carnot estimate so generally useful. Although the problem could be examined in a more sophisticated derivation, the mathematics have been kept purposefully simple in order to make the following discussion as clear as possible.

A finite time carnot refrigeration cycle (FTCRC) model of the type described below with Newton's law of cooling governing heat transfer may be the simplest refrigeration cycle model that can be used to study this problem. The FTCRC model is based on the idealized endoreversible cycle (5) in which two of the four cycle branches are defined to be reversible adiabats, and the other two are the high and low temperature isothermal branches along which finite heat fluxes couple the refrigeration cycle with external heat reservoirs.

The present analysis is focused on the FTCRC operating mode that maximizes efficiency and the cooling power properties of this maximum efficiency operating mode. other possible modes of operation might include those that maximize cooling power, minimize power consumption, or minimize irreversible entropy production. Although, for the currently studied FTCRC model, the maximum cooling power mode occurs at zero efficiency and is not physically interesting (16).

It is important to make clear that each of the 
operating modes mentioned above are unique. To construct refrigeration cycles that achieved these four modes of operation, four different refrigerators would need to be constructed. In terms of the results presented here, this means that the frequency of maximum cooling power discussed below is uniquely determined by the choice to study an FTCRC whose primary operating function is to maximize efficiency. Other modes of refrigeration cycle operation that maximize cooling power, minimize power consumption, and minimize irreversible entropy production attain different cooling power maxima at different cycling frequencies. The differences between all four of the FTCRC operating modes referred to above will be discussed in more detail in Chapter III.

A frequency dependent coefficient of performance is defined as an efficiency measure, and upon maximizing this COP, the maximum efficiency mode of refrigeration cycle operation is defined. The maximized COP converges to the global quasistatic carnot maximum in the zero cycling frequency limit and decreases monotonically to zero with increasing frequencies. In addition, the principal result of this chapter is obtained which is the maximized cop evaluated at maximum cooling power (i.e., the maximum cooling power attainable by an FTCRC operating within the maximum efficiency mode of operation). This maximized cop is given by 


$$
\varepsilon_{m}=\left(\tilde{\tau}^{2}\left(\frac{T_{H}}{T_{L}}\right)-1\right)^{-1}
$$

where $\tilde{\tau}=\sqrt{2}+1 \approx 2.41$ is the dimensionless cycle period of maximum cooling power. It is suggested that $\epsilon_{m}$ may be more useful than the quasistatic carnot cop as a theoretical bound on the thermal efficiency of certain refrigeration cycles that maximize efficiency while producing cooling power.

\section{REFRIGERATION CYCLE MODEL}

A Carnot refrigeration cycle is considered that operates at finite cycling frequencies due to thermal contact through nonzero heat fluxes with two isothermal heat reservoirs at the high and low temperatures $T_{H}$ and $T_{L}$, respectively, as shown in Figure 1. The combined system consisting of the refrigeration cycle and the two external reservoirs is isolated from all other systems. Two of the four refrigeration cycle branches are the high and low temperature isothermal branches along which heat is exchanged with the thermal reservoirs, and the other two are defined to be reversible adiabats. In addition, the working fluid is assumed to undergo only reversible transformations throughout operation of the refrigeration cycle. Therefore, changes in the entropy of the working fluid add to zero over a complete cycle. Rubin (5) was the first to refer to the 


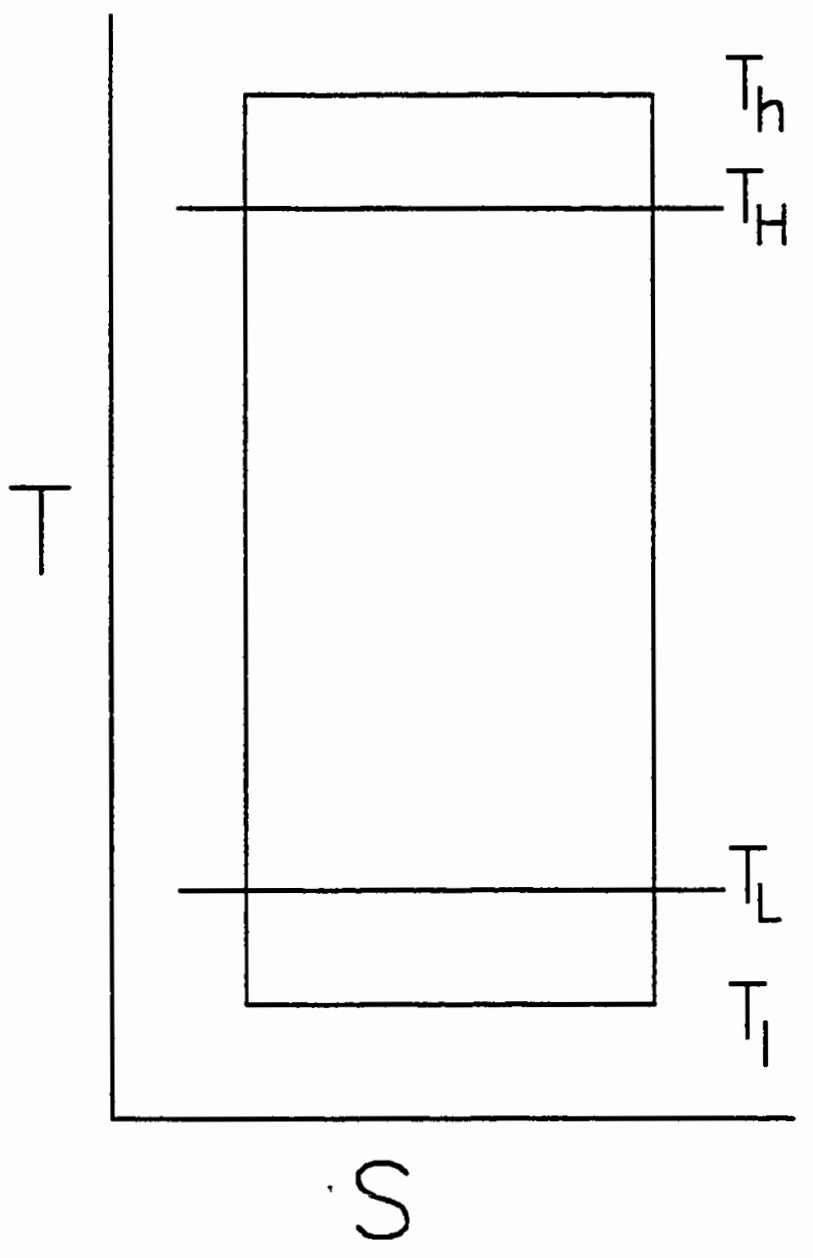

Figure 1. Temperature-entropy state space diagram of a finite time carnot refrigeration cycle. The temperatures $T_{H}$ and $T_{L}$ are the high and low temperatures of the heat reservoirs, respectively. $T_{h}$ and $T_{1}$ are the high and low temperatures of the working fluid during heat transfer, respectively. The reservoir and working fluid temperatures are ordered according to $T_{l} \leq T_{L}<T_{H} \leq T_{h}$. 
general class of cycles for which this latter assumption holds as "endoreversible". In a more physical way, endoreversibility means that the internal relaxation time of the working fluid is short compared to all other time scales associated with operation of the refrigeration cycle. Thus, the cycle defined above constitutes a carnot refrigeration cycle operating endoreversibly and generating irreversible entropy through the finite heat fluxes that connect the cycle to its high and low temperature reservoirs. For the remainder of this chapter, a refrigeration cycle of the general class depicted in Figure 1 and discussed above will be referred to as a finite time carnot refrigeration cycle (FTCRC) .

The standard assumption is made for this class of finite time cycles that the time spent along the two adiabatic branches is on a time scale consistent with minimizing heat leaks, and is negligible compared to the time taken to traverse the two heat transfer branches $(3,5,17)$. Therefore, the following approximation is used: $\tau=t_{h}+t_{1}$, where $\tau$ is the cycle period, and $t_{h}$ and $t_{1}$ are the times taken to traverse the high and low temperature heat transfer branches, respectively. As an alternative, it could have been assumed that the time spent along the two adiabats is proportional to the time spent along the heat transfer branches. In this latter case, $t_{A}=k_{A}\left(t_{h}+t_{1}\right)$, 
where $t_{A}$ is the time spent along the adiabats and $k_{A}$ is a proportionality constant. The total cycling time $\tau_{A}^{\prime}=t_{h}+t_{1}+t_{A}$ then becomes $\tau_{A}^{\prime}=k_{A}^{\prime}\left(t_{h}+t_{1}\right)$, where $k_{A}^{\prime}=k_{A}+1 . \quad$ A rescaled cycle period is defined as $\tau=\tau_{A}^{\prime} / k_{A}^{\prime}=t_{h}+t_{1}$ which is equivalent to the previous approximation in the limit of $k_{A}^{\prime}=1$. Both of these approaches have been used in studies of finite time heat engines $(2,3,5,17,18)$, and it has been shown that optimal performance is only achieved in the limit of $k_{A}^{\prime}=1$. This is also the case for the currently studied FTCRC (16). However, a certain amount of caution must be taken in interpreting this statement. Since some nonzero amount of time must be spent along the adiabatic branches, the idea of spending negligible time along the adiabats is interpreted to mean that the time scale for traversing the adiabats is small compared to the time scale for heat transfer along the isotherms but is large compared to the time scale of the internal relaxations of the working fluid. This is consistent with the physical interpretation others have given as to the nature of endoreversible cycles $(5,18)$. Heat fluxes between the working fluid of the FTCRC and the heat reservoirs at the temperatures $T_{R}=T_{H}$ or $T_{R}=T_{L}$ are represented by Newton's law of cooling which is given by 


$$
\dot{Q}=\frac{d Q}{d t}=h\left(T_{R}-T_{w}\right)
$$

where the constant $h>0$ represents the heat transfer coefficient of the system and it is assumed that the same heat transfer coefficient characterizes both heat transfer branches (i.e., "symmetric heat transfer"). Also, $T_{w}$ represents the temperature of the working fluid during high and low temperature heat transfer, and can be either $T_{w}=T_{h}$ or $T_{\mathrm{w}}=T_{1}$.

Using Eq. (2.2), the total heat transferred over either the high or low temperature branches is given by

$$
Q=\int_{0}^{t_{w}} \dot{Q} d t=\int_{0}^{t_{w}} h\left(T_{R}-T_{w}\right) d t,
$$

where $t_{w}=t_{h}$ or $t_{w}=t_{1}$ is the time taken to traverse either the high or low temperature heat transfer branch, respectively, $Q=Q_{h}<0$ for the heat rejected to the high temperature reservoir from the working fluid, and $Q=Q_{1}>0$ for the heat absorbed from the low temperature external reservoir into the working fluid. In addition, as has been done in certain studies of finite time heat engines (3), heat transfer into and out of the FTCRC is constrained in such a way that the magnitude of the entropy change in the working fluid over both heat transfer branches is $\Delta S=$ constant. Using Eq. (2.2), actual entropy changes in 
the working fluid during high and low temperature heat transfer are given by

$$
\Delta S_{\mathrm{w}}=\int_{0}^{t_{w}} \frac{\dot{Q} d t}{T_{w}}=\int_{0}^{t_{w}} \frac{h\left(T_{R}-T_{w}\right) d t}{T_{w}},
$$

where $\Delta S_{w}=\Delta S_{h}=-\Delta S$ and $\Delta S_{w}=\Delta S_{1}=\Delta S$, over the high and low temperature branches, respectively. Also, $\Delta S_{h}+\Delta S_{1}=0$ which satisfies the condition that the FTCRC operate endoreversibly.

Using Eq. (2.4), the temperature $T_{1}$ of the working fluid while heat is absorbed from the low temperature heat reservoir in the time $t_{1}$ and for the entropy change $\Delta S$ is

$$
T_{1}=T_{L}\left(\frac{\tilde{E}_{1}}{\tilde{t}_{1}+1}\right)
$$

where the dimensionless time $\tilde{t}_{1}=t_{1} h / \Delta S$ is used for mathematical convenience. Using Eqs. (2.3) and (2.5), the total heat absorbed into the working fluid from the low temperature reservoir is

$$
Q_{1}\left(\tilde{t}_{1}\right)=\frac{\Delta S T_{L} \tilde{t}_{1}}{\tilde{t}_{1}+1}
$$

Similarly, the temperature $T_{h}$ of the working fluid while heat is exhausted to the high temperature reservoir in time $t_{h}$ and for the entropy change $-\Delta S$ is 


$$
T_{h}=T_{H}\left(\frac{\tilde{t_{h}}}{\tilde{t}_{h}-1}\right),
$$

and therefore, the total heat exhausted to the high temperature heat reservoir is

$$
Q_{h}\left(\tilde{E}_{h}\right)=\frac{-\Delta S T_{H} \tilde{t}_{h}}{\tilde{t}_{h}-1}
$$

where again, a dimensionless time $\tilde{t}_{h}=t_{h} h / \Delta S$ has been introduced. It should be pointed out here that in using the dimensionless times $\tilde{E}_{h}$ and $E_{1}$ in the forthcoming optimization, the results obtained are the same as if the unscaled branch times $t_{h}$ and $t_{1}$ were used.

The present generalization of the reversible carnot refrigeration cycle to include only the irreversible entropy production due to finite heat flows to and from the external reservoirs is the refrigeration cycle analog to the finite time carnot engine cycle originally examined by curzon and Ahlborn (2) and others $(3,5)$.

\section{MAXIMUM EFFICIENCY}

In this section, the efficiency of the FTCRC is maximized. In addition, the "maximum efficiency operating mode" is defined and the efficiency at maximum cooling power within the maximum efficiency mode of operation is 
evaluated.

The efficiency of the FTCRC will be measured by a coefficient of performance (COP) given in the usual way (20) except that the work and heat are time dependent. Therefore, the COP is given by:

$$
\epsilon\left(E_{h}, E_{1}\right)=\frac{Q_{1}\left(\tilde{E}_{1}\right)}{-W_{n \theta t}\left(\tilde{t}_{h}, \tilde{E}_{1}\right)}=\left(\frac{1}{X\left(E_{h}, \tilde{E}_{1}\right)}-1\right)^{-1},
$$

where the quantity $x\left(\tilde{E}_{h}, \tilde{t}_{1}\right)=Q_{1}\left(\tilde{E}_{1}\right) /\left(-Q_{h}\left(\tilde{E}_{h}\right)\right)>0$, has been introduced. In addition, maximizing $X\left(E_{h}, \tilde{E}_{1}\right)$ is equivalent to maximizing $\epsilon$. This last statement is true because $d \epsilon / d X>0$ and $d^{2} \epsilon / d X^{2}<0$. Therefore, in order to simplify the mathematics somewhat, $x\left(\tilde{E}_{h}, \tilde{t}_{1}\right)$ will be maximized instead of maximizing $\epsilon$ directly. Using Eqs. (2.6), (2.8), and $(2.9)$,

$$
x\left(\tilde{t}_{h}, \tilde{t}_{1}\right)=\frac{\tilde{t}_{1}\left(\tilde{t}_{h}-1\right)}{\theta \tilde{t}_{h}\left(\tilde{t}_{1}+1\right)}
$$

where $\theta=T_{H} / T_{L}$.

The quantity $x\left(\tilde{t}_{h}, \tilde{t}_{1}\right)$ is maximized by obtaining the optimal relative ratio of cycle time $I=\tilde{E}_{h} / \tilde{t}_{1}$ that is devoted to each of the heat transfer branches while maintaining a constant cycle period $\tilde{\tau}=\tilde{t}_{h}+\tilde{t}_{1}$. In terms of $r$, the times devoted to low and high temperature heat 
transfer are, respectively,

$$
\tilde{t}_{h}=\tilde{\tau} \frac{r}{r+1}
$$

and

$$
\tilde{t}_{1}=\tilde{\tau} \frac{1}{r+1}
$$

In terms of $r, x\left(\tilde{t}_{h}, \tilde{t}_{1}\right)$ is given by

$$
X(r)=\frac{(r \tilde{\tau}-r-1)}{\theta r(\tilde{\tau}+I+1)}
$$

Finally, the optimum $r$ obtained from Eq. (2.12) by straightforward differentiation is

$$
r=\frac{(\tilde{\tau}+1)}{(\tilde{\tau}-1)}
$$

Using the optimum $r$ from Eq. (2.13) in (2.11a) and (2.11b), it is found that the optimal relative distribution of cycle time devoted to heat transfer that maximizes both $X\left(\tilde{t}_{h}, \tilde{t}_{l}\right)$ and $\epsilon$ is

$$
\tilde{t}_{1}=\frac{1}{2}(\tilde{\tau}-1),
$$

and

$$
\tilde{t}_{h}=\frac{1}{2}(\tilde{\tau}+1)
$$

Substituting the optimal expressions for $\tilde{t}_{h}$ and $\tilde{t}_{1}$ into Eq. (2.12) and using the resulting expression for $x\left(\tilde{t}_{h}, \tilde{t}_{1}\right)$ in 
Eq. (2.9) yields the following expression for the COP:

$$
\epsilon(\tilde{f})=\left(\frac{T_{H}}{T_{L}}\left(\frac{1+\tilde{f}}{1-\tilde{f}}\right)^{2}-1\right)^{-1}
$$

where $\tilde{f}=1 / \tilde{\tau}$ defines a reduced cycling frequency. Thus, Eq. (2.15) gives the COP of an FTCRC cycling in a way such that the highest possible efficiency is maintained at all cycling frequencies.

It is often useful to know the efficiency of a refrigeration cycle relative to the global maximum quasistatic carnot COP. Therefore, a reduced COP (RCOP) $\eta(f)=\epsilon(f) / \epsilon_{0}$ is defined, where $\epsilon_{0}=\left(\left(T_{H} / T_{L}\right)-1\right)^{-1}$ is the quasistatic Carnot COP. Thus, the RCOP for an FTCRC is given by

$$
\eta(f)=\frac{\left(T_{H} / T_{L}-I\right)}{\left(\frac{T_{H}}{T_{L}}\left(\frac{1+\tilde{f}}{1-\tilde{f}}\right)^{2}-1\right)}
$$

Due to the nature of the FTCRC model in which heat transfer occurs at a finite rate, the efficiencies of Eqs. (2.15) and (2.16) differ from the quasistatic carnot cop in at least two significant ways. First, they are both dependent on the irreversible heat transfer parameter, $h$, and cycling frequency, $f$, through the dimensionless frequency $f=f \Delta S / h$. Second, at finite cycling 
frequencies, $\epsilon(\tilde{f})$ and $\eta(\tilde{f})$ decrease monotonically to zero with increasing cycling frequency, but do converge to their respective global quasistatic Carnot maxima in the limit of $\tilde{f}=0$. In order to illustrate these features, $\eta(\tilde{f})$ is shown as a function of the reduced cycling frequency in Figure 2 .

\section{Efficiency at Maximum Cooling Power}

The cooling power over one cycle is defined to be

$$
P_{c}(\tilde{f})=Q_{1}\left(\tilde{t}_{1}\right) \hat{f} \text {. }
$$

Using Eqs. (2.7) and (2.14a), the cooling power of an FTCRC operating within the maximum efficiency mode is given by

$$
P_{c}(\tilde{f})=\frac{h T_{L} \tilde{f}(1-\tilde{f})}{(1+\tilde{f})} .
$$

As shown in Figure 2, a maximum in the cooling power exists at the dimensionless frequency

$$
f_{m}=\sqrt{2}-1
$$

and that the maximum cooling power is given by

$$
P_{c}=h T_{L}(3-2 \sqrt{2}) \text {. }
$$

Evaluating the COP of Eq. (2.14) at the frequency of maximum cooling power, the following simple expression is obtained:

$$
\epsilon_{m}=\left(\tilde{\tau}_{m}^{2}\left(\frac{T_{H}}{T_{L}}\right)-1\right)^{-1},
$$




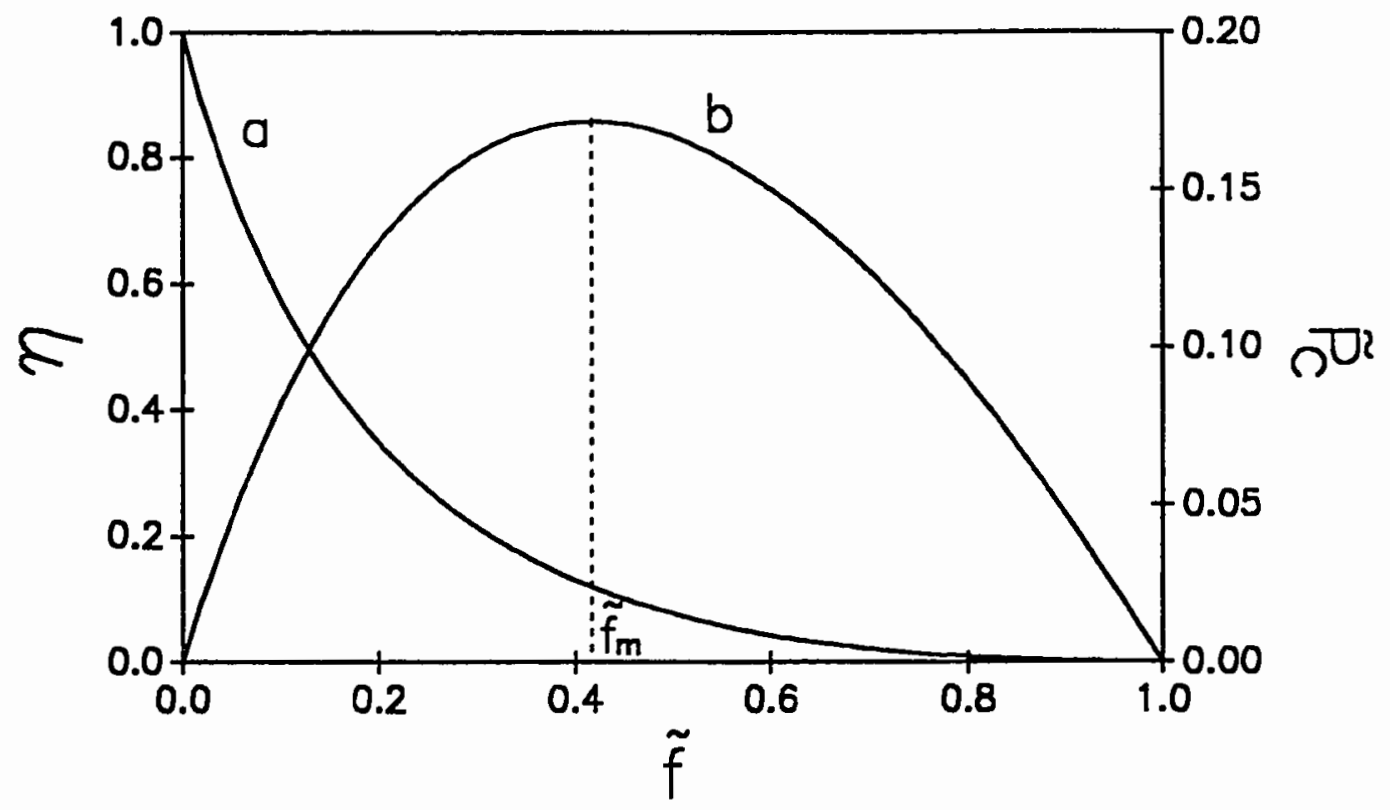

Figure 2. Curve a: reduced coefficient of performance, $\eta$, and curve $b$ : reduced cooling Power, $\tilde{P}_{c}$, as functions of the reduced cycling frequency $\tilde{f}$. The reduced frequency $\tilde{f}_{m}=\sqrt{2}-1$ is the frequency of maximum cooling power within the maximum efficiency mode of operation $(\theta=3.0)$. 
where $\tilde{\tau}_{m}=1 / \tilde{f}_{m}=\sqrt{2}+1 \propto 2.41$ is the dimensionless cycle period at maximum cooling power. As shown in Figure 3, the quasistatic Carnot COP and the COP given by Eq. (2.21) are both monotonically decreasing functions of the temperature ratio $T_{H} / T_{L}$. Although in the limit of $T_{H} / T_{L}=1$, the carnot COP diverges whereas the COP of the FTCRC remains finite. This difference may be understood by referring to the definition of the COP given in Eq. (2.9), where $\epsilon=Q_{1} /\left(-W_{n e t}\right)$. The cop for a quasistatic carnot refrigeration cycle diverges in the $T_{H} / T_{L}=1$ limit for $Q_{1} \neq 0$ because for the Carnot cycle, $W_{\text {net }}=0$ if $T_{H}=T_{L}$. However, the COP for an FTCRC producing cooling power (i.e., $Q_{1} / \tau \neq 0$ ) remains finite even in the limit of $T_{H} / T_{L}=1$ because $W_{\text {net }} \neq 0$ for an FTCRC producing cooling power regardless of the temperature ratio $T_{H} / T_{L}$ of the heat reservoirs.

Using the quasistatic Carnot COP, the RCOP at maximum cooling power within the maximum efficiency operating mode is given by

$$
\eta_{m}=\frac{\left(T_{H} / T_{L}-1\right)}{\left(\tilde{\tau}_{m}^{2}\left(\frac{T_{H}}{T_{L}}\right)-1\right)} .
$$

The COP, $\epsilon_{m}$, given by Eq. (2.21) and the RCOP, $\eta_{m}$, 


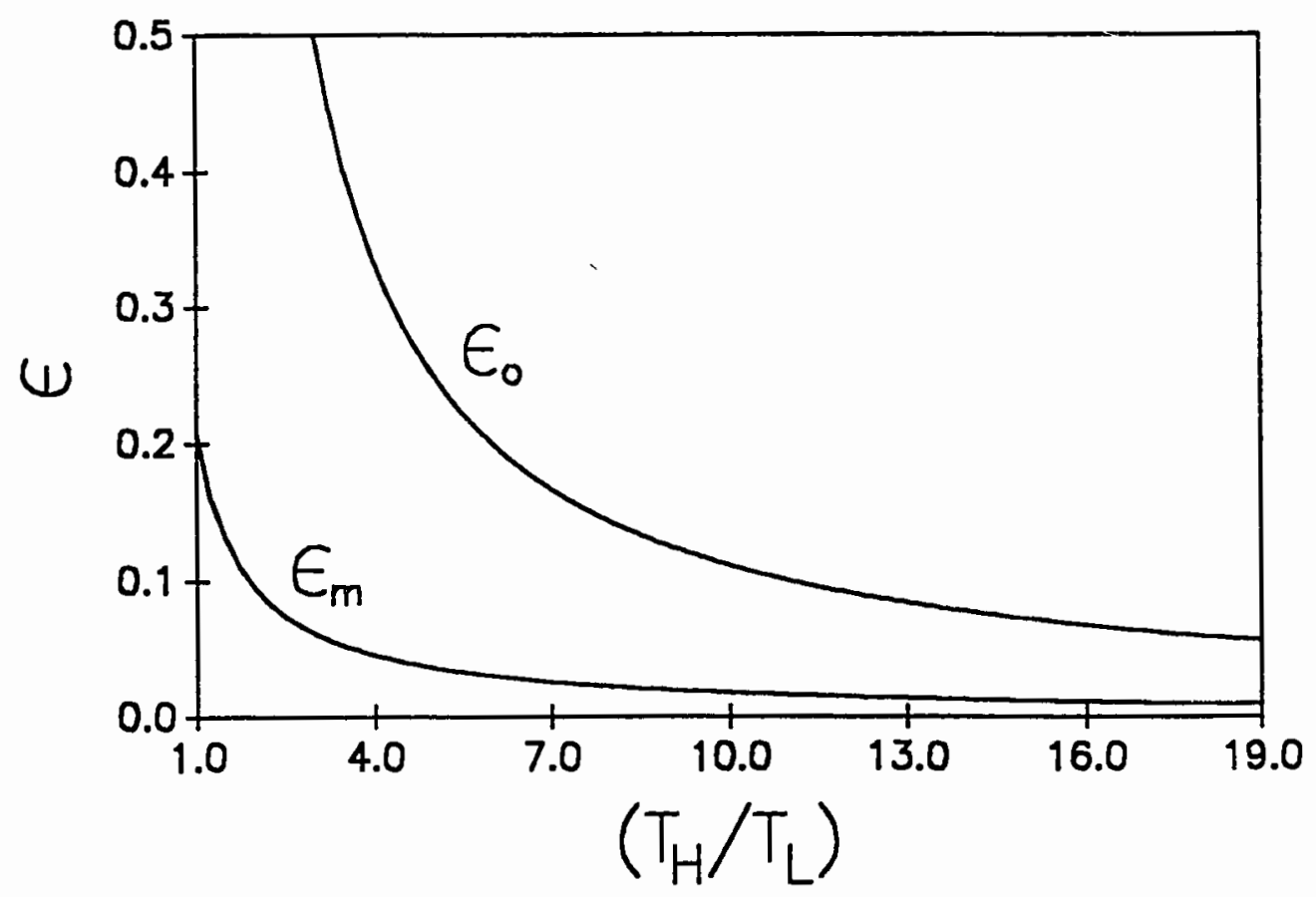

Figure 3. Quasistatic Carnot coefficient of performance, $\epsilon_{o}$, and finite time coefficient of performance at maximum cooling power, $\epsilon_{m}$, vs $T_{H} / T_{L}$ the thermal reservoir temperature ratio. 
given by Eq. (2.22) are the principal results of this chapter, and these coefficients of performance constitute new theoretical bounds on the thermal efficiency of certain refrigeration cycles that maximize efficiency while producing cooling power. In addition, $\epsilon_{m}$ and $\eta_{m}$ depend only on the temperature ratio $T_{H} / T_{L}$ and are independent of the cycling frequency and the heat transfer properties of the FTCRC. In this way, like $\eta_{C A}$ the curzon-Ahlborn efficiency of Eq. (1.2), $\epsilon_{m}$ and $\eta_{m}$ still retain much of the inherent simplicity which made the original carnot cop so generally useful. Also, $\epsilon_{m}$ and $\eta_{m}$ meet the criteria $\epsilon_{m} \leq \epsilon_{o}$, where $\epsilon_{o}$ is the quasistatic carnot COP, and $\eta_{m} \leq \eta_{0}=1$, where $\eta_{0}$ is the quasistatic carnot RCOP. Because $\varepsilon_{m}$ is most similar in form to the classic quasistatic Carnot $\operatorname{COP}, \epsilon_{m}$ is the refrigeration cycle analog to the Curzon-Ahlborn efficiency for heat engines operating at maximum power.

In Table I, the new bound on refrigeration cycle efficiency $\varepsilon_{m}$ is compared to the quasistatic carnot bound. It is important to stress that the intention is not to make a direct comparison with the experimental data because the purpose of this work is not to predict the efficiency of refrigeration cycles. Rather, interest lies in establishing bounds on efficiency that are better than the 


\section{TABLE I}

COMPARISON OF THE QUASISTATIC CARNOT COP

WITH THE COP AT FINITE COOLING POWER

OF A FINITE TIME CARNOT REFRIGERATION CYCLE

\begin{tabular}{|c|c|c|c|c|}
\hline $\begin{array}{l}\text { Refrigeration } \\
\text { Cycle }(20,21)\end{array}$ & $\mathrm{T}_{\mathrm{H}} / \mathrm{T}_{\mathrm{L}}$ & $\begin{array}{c}\epsilon_{\circ} \\
\text { (Carnot) }\end{array}$ & $\left.{ }_{\left(\mathrm{Eq} \cdot \mathrm{f}_{\mathrm{m}}\right.} 2.21\right)$ & $\begin{array}{c}\epsilon \\
\text { (Observed) }\end{array}$ \\
\hline stirling & 15 & 0.0714 & 0.0116 & 0.0021 \\
\hline $\begin{array}{l}\text { Gifford- } \\
\text { McMahon }\end{array}$ & 15 & 0.0714 & 0.0116 & 0.0009 \\
\hline Brayton & 15 & 0.0714 & 0.0116 & 0.0008 \\
\hline $\begin{array}{l}\text { Joule- } \\
\text { Thompson }\end{array}$ & 15 & 0.0714 & 0.0116 & 0.0005 \\
\hline $\begin{array}{l}\text { Reciprocating } \\
\text { Magnetic } \\
(* \mathrm{~B}=2 \mathrm{~T})\end{array}$ & 2.21 & 0.8264 & 0.0842 & 0.0777 \\
\hline$(B=5 T)$ & 2.00 & 1.0000 & 0.0938 & 0.0630 \\
\hline$(B=5 T)$ & 2.21 & 0.8264 & 0.0842 & 0.0355 \\
\hline
\end{tabular}

* "B" represents the externally applied magnetic field. 
quasistatic Carnot bounds. For a given experiment, say the stirling cycle where $T_{H} / T_{L}=15$, the experiment shows an efficiency of $\epsilon=0.0021$. The quasistatic carnot result is $\epsilon_{o}=0.0714$. Our new bound is $\epsilon_{m}=0.0116$. In this example, the new bound is lower than the carnot bound by more than a factor of 6 , and yet still bounds the experimental stirling cycle efficiency. In fact, $\varepsilon_{m}$ functions as a new bound in each of the cases shown in Table I. Therefore, based on this albeit cursory sampling of data, it is encouraging to think that the above results may indeed provide an improved bound on the thermal efficiency of certain refrigeration cycles.

\section{SUMMARY AND CONCLUSION}

A simple model for a class of endoreversible finite time Carnot refrigeration cycles has been optimized such that, in contrast to the traditional quasistatic carnot refrigeration cycle, operation proceeds irreversibly over a continuous range of cycling times and produces finite cooling power. Specifically, a refrigeration cycle model has been optimized to maximize thermal efficiency and such an optimized refrigeration cycle has been defined to operate in the maximum efficiency mode. A similar refrigeration cycle has been examined elsewhere (22) and the results are 
based on somewhat different assumptions. The principal result of this chapter has been given in Eq. (2.21), where the coefficient of performance at the maximum cooling power for a finite time carnot refrigeration cycle operating within the maximum efficiency mode has been obtained. This latter coefficient of performance may be more useful than the quasistatic carnot coefficient of performance as an estimate of the bound on thermal efficiency for certain refrigeration cycles that maximize efficiency while producing cooling power. 
CHAPTER III

\author{
OPTIMAL OPERATING MODES OF A FINITE \\ TIME REFRIGERATION CYCLE
}

INTRODUCTION

The focus of Chapter III is on a generalized finite time refrigeration cycle model which is an extension of the finite time Carnot refrigeration cycle model studied in chapter II. The basis of the refrigeration cycle model is still the idealized endoreversible cycle (5) with Newton's law heat fluxes coupling external isothermal reservoirs to the working fluid. The finite time carnot refrigeration cycle model of Chapter II has been defined so that heat transfer occurs isothermally. In the present study of a more generalized refrigeration cycle model, isothermal heat transfer is not assumed.

of main interest is in obtaining the four distinct optimal refrigeration cycle operating modes (i.e., ways of cycling the refrigeration cycle) that extremize the following objective functions: thermal efficiency, cooling power, power production, and irreversible entropy production. It is found that regardless of the objective function being considered, optimal operating modes are attained when heat transfer occurs isothermally and when the 
adiabatic branches are traversed instantaneously. The optimal cycle is a finite time carnot refrigeration cycle (FTCRC) which is the refrigeration cycle analog to the finite time carnot engine first studied by curzon and Ahlborn (2), Salamon (4), Rubin (5), and others $(8,9)$. The four FTCRC optimizations are performed that maximize thermal efficiency and cooling power, and minimize power consumption and irreversible entropy production. To examine the efficiency of these four operating modes, a three dimensional efficiency space is defined. Paths within the efficiency space that correspond to possible refrigeration cycle modes of operation are referred to as efficiency trajectories. Using the standard coefficient of performance (COP) as an efficiency measure and employing the extended Kuhn-Tucker optimization conditions (12), a globally optimum efficiency trajectory is shown to exist within the efficiency space and that this optimum trajectory defines the FTCRC operating mode that maximizes efficiency. In addition, efficiency trajectories representing the latter three operating modes referred to above are also shown within the efficiency space. The location within the efficiency space of these three additional efficiency trajectories reflects the decreased efficiency of their respective operating modes relative to the maximum efficiency mode of operation.

In addition to being maximally efficient, the maximum 
efficiency mode generates more cooling power at nonzero efficiency than any of the other optimal modes. The maximum cooling power mode generates the greatest cooling power of the four optimal modes considered but at zero efficiency, and therefore, the maximum cooling power mode is considered to be a degenerate operating mode. It should also be noted that in the context of the present finite time analysis, the quasistatic refrigeration cycle operating mode is also considered to be a degenerate FTCRC mode of operation. This last statement stems from the fact that although the quasistatic operating mode attains the global maximum carnot efficiency, refrigeration cycles operating in the quasistatic extreme cycle infinitely slowly and consequently produce no cooling power.

Expanding the time dependent coefficients of performance of the nondegenerate operating modes to first order in cycling frequency, the first order cycling frequency correction to the quasistatic Carnot cop are obtained for operating modes that maximize thermal efficiency, minimize power consumption, and minimize irreversible entropy production, respectively. In the special limiting symmetric case of equal heat transfer coefficients at the high and low temperatures, the maximum efficiency and minimum irreversible entropy production operating modes are equally efficient. The minimum power consumption mode is somewhat less efficient than these 
former two modes, and the maximum cooling power mode operates at zero efficiency.

Finally, simple analytic expressions are obtained for the coefficients of performance at maximum cooling power within each of the optimal operating modes. In the symmetric heat transfer limit, a general limiting efficiency obtained through a much less general analysis in chapter II is recovered. It is found that for Newton's law heat transfer, the FTCRC COP at maximum cooling power within the maximum efficiency operating mode is given by

$$
e_{m}=\left(\tilde{\tau}_{m}^{2}\left(\frac{T_{H}}{T_{L}}\right)-1\right)^{-1}
$$

where $\tilde{\tau}_{m}=\sqrt{2}+1 \propto 2.41$ is the dimensionless cycle period of maximum cooling power.

\section{REFRIGERATION CYCLE MODEL}

A refrigeration cycle operating at nonzero cycling frequencies is considered that is in thermal contact with two isothermal heat reservoirs with the high and low temperatures $T_{H}$ and $T_{L}$, respectively, as shown in Figure 4. Two of the four cycle branches are defined to be reversible adiabats, and the other two are the high and low temperature branches along which heat is exchanged with the external reservoirs. The combined system consisting of the refrigeration cycle and the two external reservoirs is 


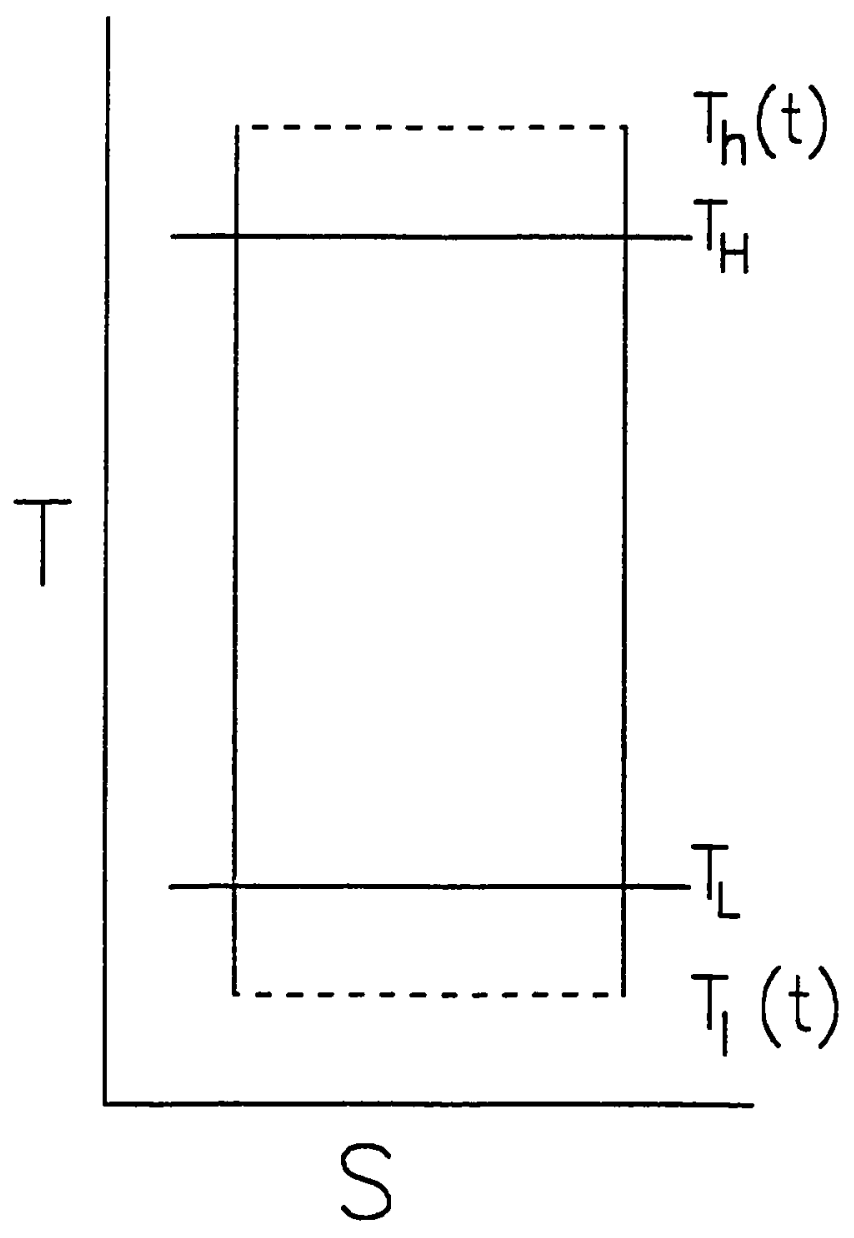

Figure 4. Temperature-entropy state space diagram of a finite time refrigeration cycle. The temperatures $T_{H}$ and $T_{L}$ are the high and low temperatures of the heat reservoirs, respectively. $T_{h}$ and $T_{1}$ are the high and low temperatures of the working fluid during heat transfer, respectively. The reservoir and working fluid temperatures are ordered according to $T_{1} \leqslant T_{L}<T_{H} \leqslant T_{h}$. 
isolated from all other systems.

More precisely, the following set of assumptions define the refrigeration cycle model:

1. The working fluid undergoes only reversible transformations throughout operation of the refrigeration cycle, and consequently, the sum of the entropy changes in the working fluid over one cycle add to zero, i.e.,

$$
\int_{0}^{\tau} d S=0
$$

where $\tau$ is the cycle period. Rubin (4) was the first to refer to a cycle for which this assumption holds as endoreversible. More physically, endoreversibility means that the internal relaxation time of the working fluid is short compared to the time scales of other cycle processes.

2. Heat fluxes between the working fluid of the refrigeration cycle and the external reservoirs are assumed to be governed by Newton's law of cooling which is given by

$$
\dot{Q}=\frac{d Q}{d t}=h\left(T_{R}-T_{w}\right),
$$

where $T_{R}$ represents the temperature of the external reservoirs and can be either $T_{H}$ or $T_{L}$. The constant $h$ represents the heat transfer coefficient, where $h=B$ or $h=\alpha(\alpha, B>0)$ along the high and low temperature heat 
transfer branches, respectively. $T_{w}(t)$ represents the temperature of the working fluid at time $t$ and can be either $T_{h}(t)$ or $T_{1}(t)$, where $T_{h}(t)$ and $T_{1}(t)$ are the high and low temperatures of the working fluid during heat transfer, respectively. Since $T_{l}(t) \leq T_{L}<T_{H} \leq T_{h}(t)$, $\dot{Q}=\dot{Q}_{1}>0$ for heat flux into the working fluid along the low temperature branch and $\dot{Q}=\dot{Q}_{h}<0$ for heat flux out of the working fluid along the high temperature branch.

3. The heat flux between the working fluid and the external reservoirs is the only source of irreversibility within the refrigeration cycle-reservoir system. other sources of irreversible entropy production, such as friction, hysteresis losses in magnetic systems, and viscous effects are not considered in this analysis.

4. Heat transferred over the high and low temperature branches is constrained to produce the absolute entropy change in the working fluid given by

$$
\Delta S=\int_{0}^{t_{w}} \frac{|\dot{Q}|}{T_{w}(t)} d t=\int_{0}^{t_{w}} \frac{\left|h\left(T_{R}-T_{w}\right)\right|}{T_{w}(t)} d t=\text { const. }
$$

where $t_{w}=t_{h}$ and $t_{w}=t_{I}$ are the times taken to traverse the high or low temperature heat transfer branches, respectively. In addition, $\Delta S_{h}=-\Delta S$ and $\Delta S_{1}=\Delta S$ are the 
entropy changes in the working fluid during high and low temperature heat transfer, and $\Delta S_{h}+\Delta S_{1}=0$ which satisfies assumption 1. As will be shown below, using Eq. (3.4) enables us to obtain analytic expressions for $T_{h}$ and $T_{1}$ in terms of $t_{h}$ and $t_{1}$ without resorting to a specific equation of state for the working fluid. Not having to use a specific equation of state for the working fluid is key in attempting to obtain results that exhibit general properties of a large class of refrigeration cycles.

Finally, the total heat transfer over either the high or low temperature branches using Eq. (3.3) may be expressed as

$$
Q_{w}=\int_{0}^{t_{w}} \dot{Q} d t=\int_{0}^{t_{w}} h\left(T_{R}-T_{w}\right) d t,
$$

where $Q=Q_{h}<0$ for the heat rejected to the high temperature reservoir from the working fluid, and $Q=Q_{1}>0$ for the heat absorbed from the low temperature external reservoir into the working fluid.

\section{OPTIMIZATION OF CYCLE BRANCHES}

The temperature of the working fluid as a function of time that extremizes efficiency, cooling power, power consumption, and irreversible entropy production is obtained in this section. First, it is shown that these four objective functions are extremized when the magnitude of the 
heat rejected to the high temperature reservoir is minimized and when the heat absorbed from the low temperature reservoir is maximized. It is then shown that both heats are extremized when heat transfer occurs isothermally and when the adiabatic branches are traversed instantaneously. Thus the time path the temperature of the working fluid follows to extremize efficiency, cooling power, power consumption, and irreversible entropy production is such that the working fluid temperature remains isothermal during heat transfer and changes instantaneously between $T_{h}$ and $T_{l}$ along the adiabatic branches.

\section{Objective Functions}

The objective functions will now be defined in terms of $Q_{h}, Q_{1}$, and the cycle period $\tau=t_{h}+t_{1}+t_{A}$, where $t_{A}$ is the time devoted to the adiabatic branches.

The coefficient of performance (COP) is defined in the usual way (15) as

$$
\boldsymbol{\epsilon}=\frac{Q_{1}}{-W_{\text {net }}}=\frac{Q_{1}}{-\left(Q_{h}+Q_{1}\right)},
$$

where

$$
\begin{aligned}
& \frac{\partial \epsilon}{\partial Q_{1}}=\frac{-Q_{h}}{\left(Q_{h}+Q_{1}\right)^{2}}>0, \\
& \frac{\partial^{2} \varepsilon}{\partial Q_{1}{ }^{2}}=\frac{2 Q_{h}}{\left(Q_{h}+Q_{1}\right)^{3}}<0,
\end{aligned}
$$


and

$$
\begin{aligned}
& \frac{\partial \epsilon}{\partial Q_{h}}=\frac{Q_{1}}{\left(Q_{h}+Q_{1}\right)^{2}}>0, \\
& \frac{\partial^{2} \epsilon}{\partial Q_{h}^{2}}=\frac{-2 Q_{1}}{\left(Q_{h}+Q_{1}\right)^{3}}<0 .
\end{aligned}
$$

Taking into account that $Q_{h}\left\langle 0\right.$ and $Q_{1}>0, \epsilon$ is maximized when both $Q_{h}$ and $Q_{1}$ are maximized $\left(Q_{h}>0\right.$ is minimized). In addition, the derivatives of $\varepsilon$ with respect to the ratio $0<X=-Q_{1} / Q_{h}<1$ are

$$
\frac{\partial \varepsilon}{\partial X}=\frac{1}{X^{2}}\left(\frac{1}{X}-1\right)^{-2}>0
$$

and

$$
\frac{\partial^{2} \varepsilon}{\partial X^{2}}=\frac{-2}{X^{3}}\left(\frac{1}{X}-1\right)^{-2}\left(1-\frac{1}{(1-X)}\right)<0,
$$

where

$$
\frac{\partial X}{\partial Q_{h}}=\frac{Q_{1}}{Q_{h}^{2}}>0
$$

and

$$
\frac{\partial X}{\partial Q_{1}}=\frac{-1}{Q_{h}}>0
$$

Equations (3.7a) and (3.7b) show that $€$ is maximized when $X$ is maximized, and Eqs. (3.8a) and (3.8b) show that, like $\varepsilon, X$ 
is maximized for maximum $Q_{h}\left\langle 0\right.$ and $Q_{1}>0$. Therefore, maximizing $X$ is equivalent to maximizing $\epsilon$. This result will be used to simplify the optimization of $\epsilon$. The cooling power generated by the refrigeration cycle is given by

$$
P_{c}=\frac{Q_{1}}{\tau}
$$

and

$$
\frac{\partial P_{c}}{\partial Q_{1}}=\frac{1}{\tau}>0 .
$$

Because $P_{c}$ is linear with respect to $Q_{1}$ and $\partial P_{c} / \partial Q_{1}$ is strictly greater than zero, cooling power is maximized for maximum $Q_{1}>0$.

The power consumed per cycle is defined as

$$
P=\frac{-W_{\text {net }}}{\tau}=\frac{-\left(Q_{h}+Q_{1}\right)}{\tau}
$$

where $-W_{n e t}=-\left(Q_{h}+Q_{1}\right)>0$ is the work required to complete one cycle. The changes in the power consumed per cycle with respect to changes in $Q_{h}<0$ and $Q_{1}>0$ are

$$
\frac{\partial P}{\partial Q_{1}}=\frac{-1}{\tau}<0,
$$

and

$$
\frac{\partial P}{\partial Q_{h}}=\frac{-1}{\tau}<0 .
$$


Because $P$ is linear with respect to heat transfer and the derivatives in (3.10b) and (3.10c) are strictly less than zero, power consumption is minimized when $Q_{h}$ and $Q_{1}$ are maximized.

Irreversible entropy production is given by

$$
\Delta S_{I}=-\left(\frac{Q_{h}}{T_{H}}+\frac{Q_{1}}{T_{L}}\right),
$$

where

$$
\frac{\partial \Delta S_{I}}{\partial Q_{1}}=\frac{-1}{T_{L}}<0,
$$

and

$$
\frac{\partial \Delta S_{I}}{\partial Q_{h}}=\frac{-I}{T_{H}}<0 .
$$

Because, the derivatives in (3.12a) and (3.12b) are strictly negative and $\Delta S_{I}$ varies linearly with respect to $Q_{h}$ and $\ell_{1}, \Delta S_{I}$ is minimized when both $Q_{h}$ and $Q_{1}$ are maximized.

\section{optimizing Heat Transfer}

The problem now is to obtain the temperature of the working fluid as a function of time along the heat transfer and adiabatic branches that extremizes $Q_{h}$ and $Q_{1}$.

Heat Transfer Branches. To obtain the optimum temperature of the working fluid, $T_{w}(t)$, along the heat transfer branches, the Euler-Lagrange method is used. The 
Lagrangian for this optimization, using Eqs. (3.3) and $(3.4)$, is

$$
\mathscr{L}=\dot{Q}-\lambda \frac{\dot{Q}}{T_{w}(t)}=h\left(T_{R}-T_{w}(t)\right)\left(1-\frac{\lambda}{T_{w}(t)}\right),
$$

where $\lambda$ is a Lagrange multiplier. The optimal $T_{w}(t)$

obtained from the simplified Euler-Lagrange equation, $\partial \mathscr{L} / \partial T_{w}(t)=0$, is

$$
T_{w}(t)=\sqrt{\lambda T_{R}}=\text { const. }=T_{w}>0 \text {. }
$$

The Lagrange multiplier is

$$
\lambda=\frac{T_{w}^{2}}{T_{R}}>0,
$$

and the Legendre condition is given by

$$
\frac{\partial^{2} \mathscr{L}}{\partial T_{w}^{2}}=\frac{-2 \lambda h T_{R}}{T_{w}^{3}}<0 .
$$

The free endpoint conditions (13)

$$
\left.\frac{\partial \mathscr{L}}{\partial \dot{T}_{w}}\right|_{t=0}=\left.\frac{\partial \mathscr{L}}{\partial \dot{T}_{w}}\right|_{t=t_{w}}=0
$$

are automatically satisfied. Thus, it follows from Eqs. (3.14) - (3.17) that the optimal way to transfer heat between a thermal reservoir at a fixed temperature and an endoreversible working fluid undergoing an entropy change $\Delta S_{w}$ in time $t_{w}$, is isothermally. In addition, it has been 
shown elsewhere that optimal heat transfer branches should be made up of only one continuous isotherm (3). Therefore, using Eq. (3.4), the isothermal temperature of the working fluid is given by

$$
T_{\mathrm{w}}=\frac{T_{R}}{\left(1+\frac{\Delta S_{\mathrm{w}}}{h t_{\mathrm{w}}}\right)} .
$$

To complete this variational analysis, it is pointed out that Salamon et al. (3) have shown that because the freedom exists to connect the isothermal heat transfer branches with discontinuous adiabatic transitions, it is of no concern that the solution to the present Euler-Lagrange variational problem will, in general, not fall at the temperatures $T_{w}(0)$ and $T_{w}\left(t_{w}\right)$ at the times $t=0$ and $t=t_{w}$.

Using $T_{w}$ in (3.5), the heat exhausted from the working fluid of the FTCRC into the high temperature reservoir along the high temperature heat transfer branch in time $\tilde{E}_{h}$ and for the entropy change $-\Delta S$ is given by

$$
Q_{h}\left(\tilde{t}_{h}\right)=\frac{-\Delta S T_{H} \tilde{t}_{h}}{\left(\tilde{t}_{h}-\Gamma\right)},
$$

and the heat absorbed by the working fluid from the low temperature reservoir along the low temperature isotherm in time $E_{1}$ and for the entropy change $\Delta S$ is 


$$
Q_{1}\left(\tilde{t}_{1}\right)=\frac{\Delta S T_{1} \Gamma \tilde{t}_{1}}{\left(\Gamma \tilde{E}_{1}+1\right)}
$$

The dimensionless variables

$$
\Gamma=\sqrt{\frac{\alpha}{B}}, \quad \tilde{t}_{1}=t_{1} \frac{\sqrt{\alpha \mathcal{B}}}{\Delta S}, \text { and } \quad E_{h}=t_{h} \frac{\sqrt{\alpha \mathcal{B}}}{\Delta S}
$$

have been introduced for mathematical convenience.

For the remainder of this paper, a refrigeration cycle of the type described above that operates at nonzero cycling times, and in which heat transfer occurs along two isothermal branches that are connected by two ideal adiabatic branches will be referred to as a finite time Carnot refrigeration cycle (FTCRC).

Adiabatic branches. To complete this portion of the analysis, the temperature of the working fluid as a function of time along the adiabatic branches that further optimizes $Q_{1}$ and $Q_{h}$ is obtained. This is accomplished by examining the effect on heat transfer of shifting an amount of cycling time $\Delta \tilde{t}_{A}=\Delta t_{A} \sqrt{\alpha \beta} / \Delta S$, from an adiabatic branch to a heat transfer branch while maintaining a constant total cycling time. The changes in $Q_{h}$ and $Q_{1}$ with respect to changes in the time allocated to their respective heat transfer branches are

$$
\frac{d Q_{1}}{d \tilde{t}_{1}}=\frac{\Delta S T_{L} \Gamma}{\left(\Gamma \tilde{t}_{1}+1\right)^{2}}>0,
$$


$\frac{d Q_{1}}{d \tilde{t}_{h}}=0$

$$
\frac{d Q_{h}}{d \tilde{t}_{h}}=\frac{\Delta S T_{H}}{\left(\tilde{t}_{h}-\Gamma\right)^{2}}>0,
$$

and

$$
\frac{d Q_{h}}{d \tilde{t}_{1}}=0
$$

It follows from Eqs. (3.21a), (3.21b), (3.22a), and (3.22b) that both $Q_{h}$ and $Q_{1}$ are further optimized in the $\tilde{t}_{A}=0$ limit. Thus, optimal operation of the FTCRC requires that the working fluid temperature switch instantaneously between $T_{h}$ and $T_{1}$ along the adiabatic cycle branches. Although, it must be added that physically some nonzero amount of time must be spent along the adiabatic branches. Therefore, spending zero or negligible time along the adiabats is interpreted to mean that the time scale for traversing the adiabats is small compared to the time scale for heat transfer along the isotherms but is large compared to the time scale of the internal relaxations of the working fluid.

Thus, the time path the temperature of the working fluid follows to optimize heat transfer and extremize efficiency, cooling power, power consumption, and irreversible entropy production of the FTCRC is such that 
the working fluid temperature remains isothermal during heat transfer and switches instantaneously, in the sense described above, between $T_{h}$ and $T_{1}$ along the two adiabatic branches.

OPTIMAL FTCRC OPERATING MODES

The optimal FTCRC operating modes that extremize efficiency, cooling power, power consumption, and irreversible entropy production are obtained. The extended Kuhn-Tucker optimization procedures are used (12), and the relative distribution of cycling time devoted to heat transfer along the high and low temperature heat transfer isotherms is optimized. In addition, using the solutions to the Kuhn-Tucker optimizations, the following two efficiencies are obtained within each of the four optimal FTCRC operating modes: 1) the first order correction to the quasistatic Carnot cop for nonzero cycling times and 2) the coefficients of performance at maximum cooling power.

Since the Kuhn-Tucker (KT) optimization conditions are not commonly used in physics, it is worth outlining them here.

If in the problem:

$$
\begin{aligned}
\text { Maximize: } & f(X), \\
\text { subject to: } & g_{j}(X) \leq 0, j=1,2, \ldots, \ldots, m,(3.24 \mathrm{a}) \\
& h_{k}(X)=0, k=1,2, \ldots, \ldots, 1,(3.24 \mathrm{~b})
\end{aligned}
$$


$f$ is suitably concave (convex for a minimum), and $g_{j}$ and $h_{k}$ are convex for $j=1,2, \ldots, m$, and $k=1,2, \ldots, 1$, respectively, and there exist $X^{*}$ and $\mu^{*}=\left(\mu_{1}^{*}, \mu_{2}^{*}, \ldots, \mu_{m}^{*}\right)$ which satisfy

$$
\begin{aligned}
& \left.\nabla \mathscr{L}=\nabla\left(f\left(X^{*}\right)\right)-\sum_{j} \mu_{j} g_{j}\left(X^{*}\right)-\sum_{k} \lambda_{k} h_{k}\left(X^{*}\right)\right)=0, \\
& g\left(X^{*}\right) \leq 0, \\
& \begin{array}{ll}
\mu_{j} g_{j}\left(X^{*}\right)=0, & j=1,2, \ldots, m \\
\mu_{j} \geq 0, & j=1,2, \ldots, m
\end{array}
\end{aligned}
$$

and

$$
h_{k}\left(X^{*}\right)=0, \quad k=1,2, \ldots, 1
$$

then $f(X)$ has a global maximum (minimum) at $X^{*}$, where the $\mu$ and $\lambda$ are equivalent to Lagrange multipliers.

In the present optimization, $f(X)$ may represent $\varepsilon\left(\tilde{t}_{1}, \tilde{t}_{h}\right), P_{c}\left(\tilde{t}_{1}, \tilde{t}_{h}\right), P\left(\tilde{E}_{1}, \tilde{E}_{h}\right)$, or $\Delta S_{I}\left(\tilde{E}_{1}, \tilde{t}_{h}\right)$, where these objective functions have been defined above. Proving that $\varepsilon$ and $P_{c}$ are concave functions and that $P$ and $\Delta S_{I}$ are convex is complicated. The mathematical details of these latter proofs are given in Appendix A.

The constraints defining the feasible region for optimal FTCRC operating modes are

$$
\begin{aligned}
& g_{1}=-\tilde{t}_{1} \leq 0, \\
& g_{2}=\Gamma-\tilde{t}_{h} \leq 0,
\end{aligned}
$$


and

$$
h=\tilde{\tau}-\left(\tilde{t}_{h}+\tilde{t}_{1}\right)=0 .
$$

Constraint $g_{1}$ assures that the time spent along the low temperature heat transfer branch is positive and nonzero. Constraint $g_{2}$ is necessary in order to keep $Q_{h}<0$. Constraint $h$ requires that the sum of the times devoted to heat transfer is the total cycle time. Because the set of solutions to a system of linear equalities or inequalities is a convex set (14), solutions satisfying Eqs. (3.30a) (3.30c) constitute convex sets which satisfy the extended Kuhn-Tucker convexity conditions. Therefore, because $P$ and $\Delta S_{I}$ are suitably convex, $\epsilon$ and $P_{c}$ are suitably concave, and the constraint set is suitably convex, each of the following optimizations yield global optima.

Optimizing the four objective functions with respect to the dimensionless branch times $\tilde{t}_{h}$ and $\tilde{t}_{1}$ is equivalent to optimizing with respect to $t_{h}$ and $t_{1}$, and it is straightforward to reexpress optimal results in terms of the natural branch times $t_{h}$ and $t_{1}$.

\section{Maximum Efficiency}

Using Eqs. (3.19) and (3.20), the COP of Eq. (3.6) is given by

$$
\epsilon\left(\tilde{t}_{1}, \tilde{t}_{h}\right)=\left(\frac{\theta \tilde{t}_{h}\left(\Gamma \tilde{t}_{1}+1\right)}{\Gamma \tilde{t}_{1}\left(\tilde{t}_{h}-\Gamma\right)}-1\right)^{-1}
$$




$$
=\left(\frac{1}{X\left(\tilde{t}_{1}, \tilde{t}_{h}\right)}-1\right)^{-1},
$$

where $\theta=T_{H} / T_{L}$ is the environmental temperature ratio. Using $\varepsilon\left(\tilde{E}_{1}, \tilde{E}_{h}\right)$, a three dimensional efficiency space is defined which consists of surfaces of constant $\theta$ and $\Gamma$ as shown in Figure 5. Efficiency space surfaces are composed of contours of constant cycling time which vary continuously with the ratio $\tilde{t}_{h} / \tilde{t}_{1}$ and are obtained by plotting the ordered triple $\left(\tilde{f}_{1}, \tilde{f}_{h}, \eta\left(\tilde{f}_{1}, \tilde{f}_{h}\right)\right)$ as $\tilde{E}_{h} / \tilde{E}_{1}$ varies from 0 to $\infty$ within the feasible region, where $\tilde{f}_{1}=1 / \tilde{E}_{1}, \tilde{f}_{h}=1 / \tilde{t}_{h}$, $\eta\left(\tilde{f}_{1}, \tilde{E}_{h}\right)=\varepsilon\left(\tilde{f}_{1}, \tilde{f}_{h}\right) / \epsilon_{0}$. The $\operatorname{cop} \epsilon_{0}=\left(\left(T_{H} / T_{L}\right)-1\right)^{-1}$ is the quasistatic carnot COP. Paths within the efficiency space describing possible refrigeration cycle operating modes are referred to as efficiency trajectories, and an efficiency space as defined above contains efficiency trajectories for all possible operating modes of the FTCRC considered in this analysis. Note that the efficiency trajectory identifying the maximum Carnot efficiency zero cooling power quasistatic operating mode is the degenerate trajectory given by the point $(0,0,1)$ and is obtained in the limit of $\tilde{f}_{1}=\tilde{f}_{h}=0$. This quasistatic operating mode corresponds to the first of two FTCRC degenerate operating modes. The second degenerate operating mode is the maximum cooling power mode which 


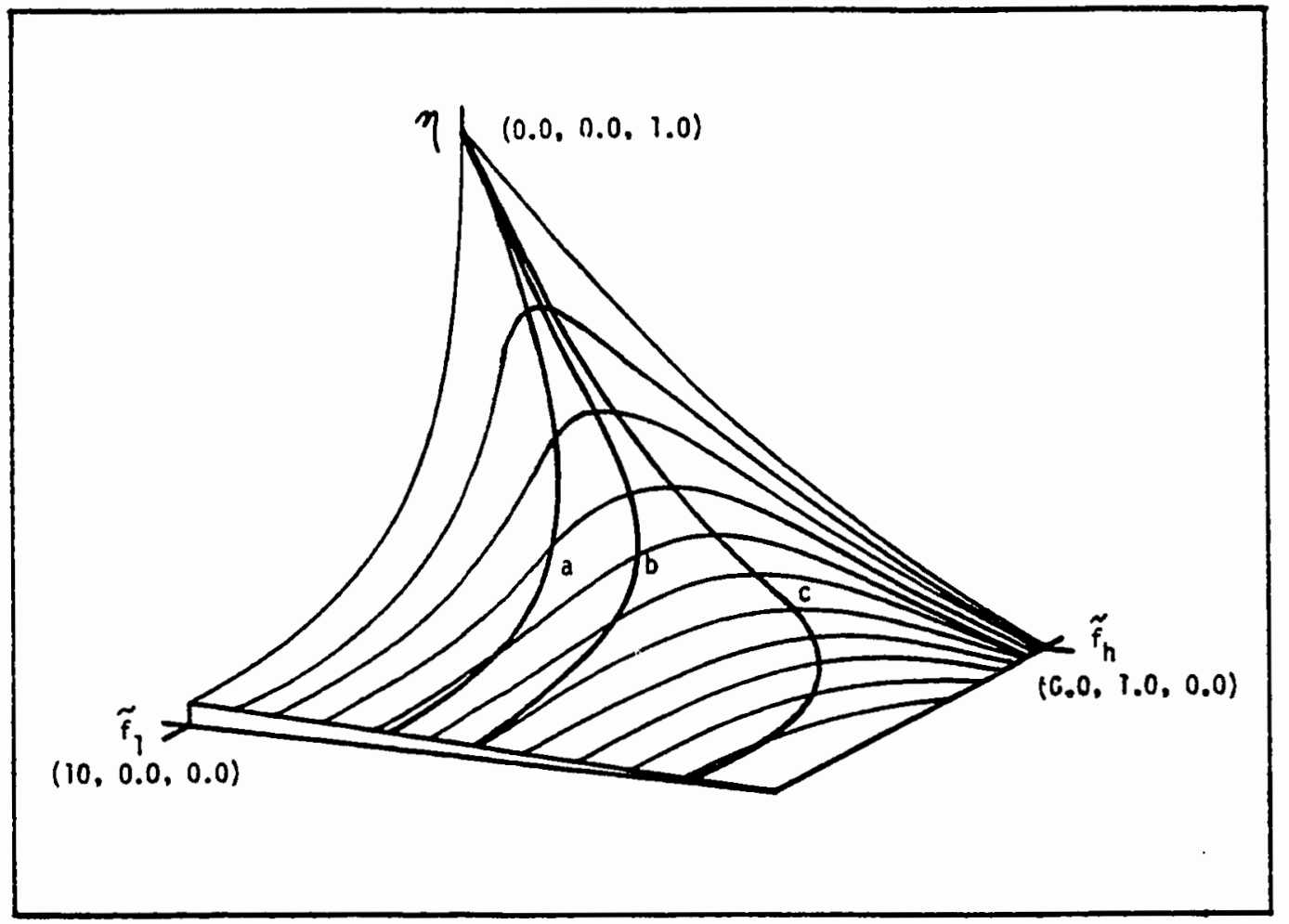

Figure 5. Three dimensional efficiency space. An efficiency space surface composed of contours of constant cycling time for an FTCRC with $\Gamma=1$ and $\theta=3$. The reduced $\operatorname{cop} \eta\left(f_{h}, f_{1}\right)=\epsilon\left(f_{h}, f_{1}\right) / \epsilon_{0}$ is plotted vs the inverse times $\tilde{E}_{h}=1 / \tilde{E}_{h}$ and $\tilde{f}_{1}=1 / \tilde{t}_{1}$ spent along the low and high temperature heat transfer branches, respectively. Efficiency trajectories are shown for the following four optimized cycling configurations: a), minimum power consumption; b), minimum irreversible entropy production; c), maximum reduced coefficient of performance. The global maximum Carnot efficiency zero cooling power quasistatic operating mode is identified by the degenerate trajectory given by the point $(0.0,0.0,1.0)$. 
produces the global maximum cooling power but at zero efficiency.

To obtain the global maximum efficiency trajectory within the three dimensional efficiency space $X\left(\tilde{t}_{1}, \tilde{t}_{h}\right)$ is maximized because it has been shown that maximizing $X\left(E_{1}, \tilde{E}_{h}\right)$ is equivalent to maximizing $\epsilon\left(\tilde{E}_{1}, \tilde{E}_{h}\right)$ directly. (In appendix $A$, it is shown that $X\left(\tilde{E}_{1}, \tilde{E}_{h}\right)$ is suitably concave over the feasible region.) To maximize $x\left(E_{1}, \tilde{t}_{h}\right)$, a modified $\mathrm{KT}$-Lagrangian is formed and is given by

$$
\mathscr{L}=\frac{\Gamma \tilde{t}_{1}\left(\tilde{t}_{h}-\Gamma\right)}{\theta \tilde{E}_{h}\left(\Gamma \tilde{E}_{1}+1\right)}-\mu_{1} \tilde{E}_{1}-\mu_{2}\left(\Gamma-\tilde{t}_{h}\right)
$$

$$
-\lambda\left(\tilde{\tau}-\left(E_{h}+E_{1}\right)\right)
$$

Solving the system of equations $\nabla \mathscr{L}=0$ and taking $\mu_{1}^{*}=\mu_{2}^{*}=0$ and $\lambda^{*}=-1$, the following is obtained:

$$
\Gamma \hat{\epsilon}_{1}\left(\Gamma \hat{\epsilon}_{1}^{*}+1\right)=\hat{\epsilon}_{h}^{*}\left(\hat{\epsilon}_{h}^{*}-\Gamma\right)
$$

Therefore, using $\tilde{\tau}=\tilde{t}_{h}+\tilde{E}_{1}$, the maximum efficiency operating mode is attained when

$$
\tilde{t}_{1}^{*}=\frac{-\tilde{\tau}+\left(\Gamma \tilde{\tau}\left(\Gamma \tilde{\tau}+1-\Gamma^{2}\right)\right)^{1 / 2}}{\left(\Gamma^{2}-1\right)},
$$

and

$$
\tilde{E}_{h}=\frac{\tilde{\tau} \Gamma^{2}-\left(\Gamma \tilde{\tau}\left(\Gamma \tilde{\tau}+1-\Gamma^{2}\right)\right)^{1 / 2}}{\left(\Gamma^{2}-1\right)},
$$


which will be real for $\tilde{\tau}>\left(\Gamma^{2}-1\right) / \Gamma$. Using $\hat{t}_{1}$ and $\hat{t}_{h}^{*}$ with the COP given in Eq. (3.31) defines the global maximum efficiency trajectory shown in Figure 5. Also shown in Figure 5 are the efficiency trajectories corresponding to the optimal FTCRC modes that extremize cooling power, power consumption, and irreversible entropy production, where these latter two optimizations have been carried out below. The efficiency trajectories of all four optimal cycling modes converge to the quasistatic Carnot maximum of $\left(\tilde{f}_{1}, \tilde{f}_{h}, \eta\right)=(0,0,1)$ in the infinite cycling time quasistatic limit.

Combining $(3.31),(3.34 \mathrm{a})$, and $(3.34 \mathrm{~b})$, the frequency dependent $C O P$ for the maximum efficiency operating mode is given by

$$
\epsilon(f)=\left(\frac{\theta\left(\Gamma^{2}-\sqrt{\xi}\right)\left(-\Gamma+\Gamma \sqrt{\xi}+\tilde{f}\left(\Gamma^{2}-1\right)\right)}{(-\Gamma \sqrt{\xi})\left(\Gamma^{2}-\sqrt{\xi}-\tilde{\Gamma}\left(\Gamma^{2}-1\right)\right)}-1\right)^{-1},
$$

where

$$
\xi=\Gamma^{2}-\tilde{f} \Gamma\left(\Gamma^{2}-1\right)
$$

Expanding Eqs. (3.35) about $F=0$, the following expression for the first order cycling frequency correction to the quasistatic carnot cop is obtained:

$$
\epsilon_{1}^{(e)} \approx \epsilon_{o}\left(1-\frac{\theta(\Gamma+1)^{2}}{\Gamma(\theta-1)} \vec{f}\right)
$$


where the superscript notation in Eq. (3.36) is introduced to distinguish the coefficients of performance of different optimal FTCRC operating modes.

In Figure $6, \epsilon(\tilde{f})$ and $\epsilon_{1}^{(e)}$ are shown as functions of cycling frequency for $\Gamma=1$ and $\theta=3.0$. Also shown are the frequency dependent coefficients of performance and their respective first order expansions for the minimum power consumption and irreversible entropy production operating modes which have been obtained below. It is found that to first order in $\vec{f}$ and for $\Gamma=1$ that the maximum efficiency and minimum irreversible entropy modes are equally efficient and that minimum power consumption mode operates somewhat less efficiently than these latter two modes. In addition, the efficiencies of these three distinct operating modes reduce monotonically to zero with increasing cycling frequency and converge to the quasistatic global carnot maximum in the $E=0$ limit. Further, the coefficients of performance shown in Figure 6 approach zero efficiency at different operating frequencies due to the different way each optimal mode requires cycling time to be distributed to the heat transfer branches. The efficiencies of the minimum power consumption and irreversible entropy production modes approach zero as $Q_{1} \rightarrow 0$ and the maximum efficiency mode approaches zero as $: W_{\text {net }}: \infty$.

To obtain the COP at maximum cooling power, for the 


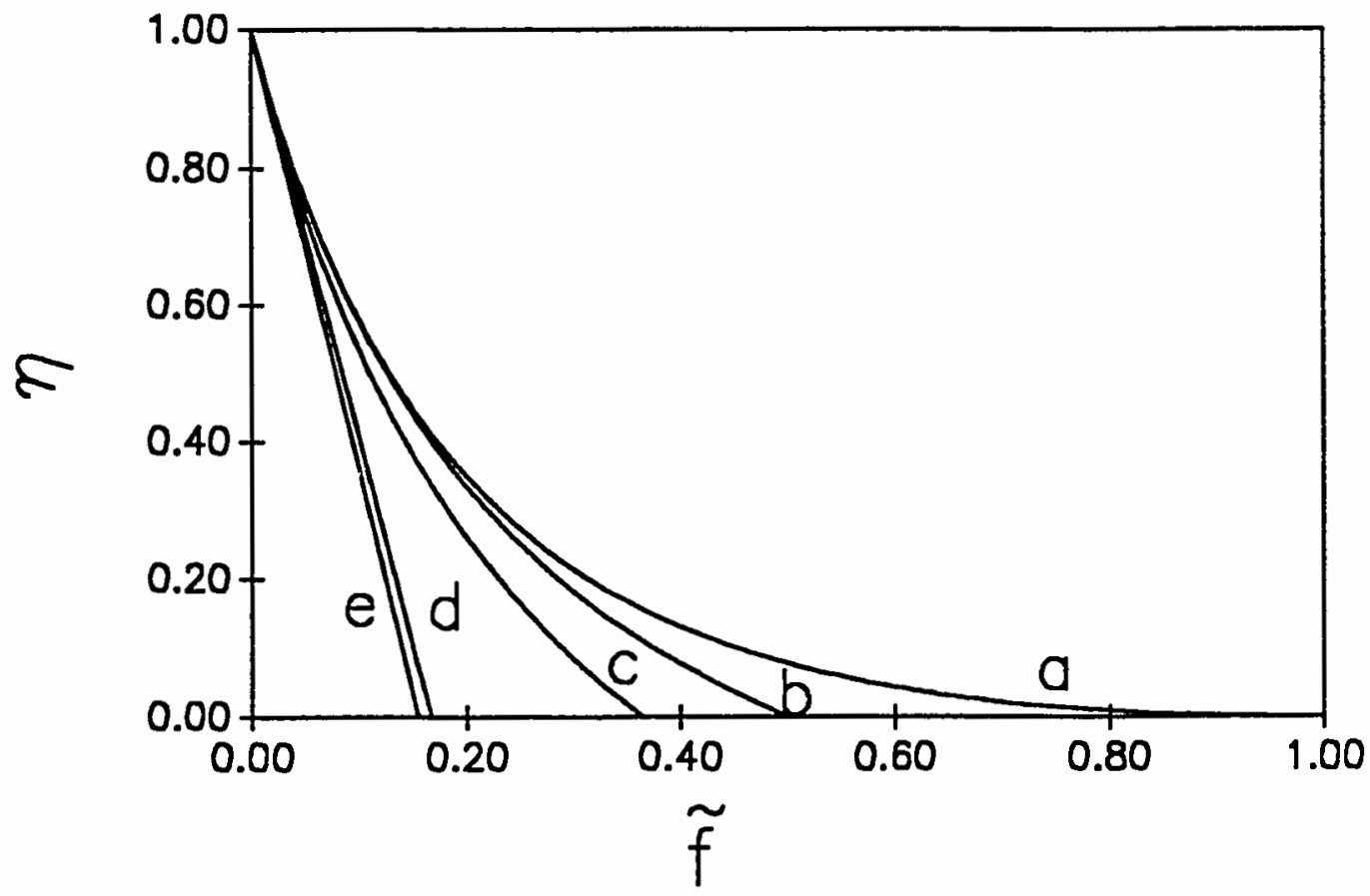

Figure 6. Reduced coefficients of performance, $\eta$, vs reduced cycling frequency, $\vec{f}$, for four optimal operating modes. Curves $a, b$, and $c$ represent the COP of the maximum efficiency, minimum power consumption, and minimum irreversible entropy production modes, respectively $(\theta=3$ and $\Gamma=1)$. Curve $d$ represents the first order expansion of the cop in terms of $f$ for both the maximum efficiency and minimum irreversible entropy production modes, and curve e represents the first order expansion of the COP for the minimum power consumption operating mode. 
maximum efficiency operating mode only, the problem is simplified to the special symmetric case of $\Gamma=1$ (i.e., $\alpha=B=h$ ) because obtaining an analytic expression for the cycling period of maximum cooling power for arbitrary values of $\Gamma$ appears to be algebraically intractable.

The cooling power of an FTCRC operating in the maximum efficiency mode with $\Gamma=1$ and using (3.9a), (3.20), and (3.34a) is given by

$$
P_{c}=\frac{h T_{L}(\tilde{\tau}-1)}{\tilde{\tau}(\tilde{\tau}+1)} \text {. }
$$

Maximum cooling power is attained when

$$
\tilde{\tau}=\tilde{\tau}_{m}=\sqrt{2}+1 \text {. }
$$

Thus, under the special symmetric limiting condition of equal heat transfer coefficients, the following simple form for the COP at maximum cooling power which has been obtained elsewhere (12) in a less mathematically general derivation:

$$
\boldsymbol{\epsilon}_{m}^{(\boldsymbol{e})}=\left(\tilde{\tau}_{m}^{2}\left(\frac{T_{H}}{T_{L}}\right)-\right)^{-1} .
$$

The maximum cooling power within the maximum efficiency operating mode is given by

$$
P_{c}=\sqrt{\alpha \mathcal{B}} T_{L}(3-2 \sqrt{2}) \text {. }
$$

\section{Maximum Cooling Power}

Cooling power is defined in Eq. (3.9a). Using the constraints given by $g_{1}, g_{2}$, and $h$, the Lagrangian for 
maximizing cooling power is

$$
\mathscr{L}=\frac{\sqrt{\alpha \mathcal{B}} T_{L} \Gamma \tilde{t}_{1}}{\tilde{\tau}\left(\Gamma \tilde{t}_{1}+1\right)}+\mu_{1} \tilde{E}_{1}-\mu_{2}\left(\Gamma-\tilde{t}_{h}\right)
$$

$$
-\lambda\left(\tilde{\tau}-\left(\tilde{t}_{h}-\tilde{t}_{1}\right)\right)
$$

The Lagrange multipliers are $\mu_{1}^{*}=0$ and $\lambda^{*}=-1$ as before, but in order to satisfy constraint $g_{2}$ and obtain a solution within the feasible region, the Kuhn-Tucker conditions require that $\mu_{2}^{*}=1$. If $\mu_{2}^{*}=0$ as in the previous case of maximizing $\epsilon, \tilde{t}_{h}=\tilde{\tau}=0$ is obtained which violates $g_{2}$. Using $\mu_{2}^{*}=1$,

$$
\epsilon_{h}=\Gamma \text {, }
$$

and

$$
\hat{t}_{1}=\tilde{\tau}-\Gamma \text {. }
$$

Using (3.42) and (3.43), it is found that the COP within the maximum cooling power operating mode is given by

$$
\varepsilon^{\left(P_{c}\right)}=0 \text {. }
$$

The efficiency is zero because infinite work input is required to operate the FTCRC in the maximum cooling power mode. This last statement is true because as $\tilde{t}_{h} \rightarrow \Gamma, T_{H} \rightarrow \infty$ and $\left|\ell_{h}\right|-\infty$, and consequently, $-W_{n e t}=\left|Q_{h}+Q_{1}\right|-\infty$ and $\epsilon=Q_{1} /\left(-W_{\text {net }}\right)=0$. 
For the moment, the fact that the maximum cooling power mode operates at zero efficiency is disregarded in order to establish an upper bound on the cooling power of the FTCRC. Using Eqs. (3.42) and (3.43), the cooling power within the maximum cooling power operating mode is given by

$$
P_{c}=\frac{\sqrt{\alpha B} T_{L} \Gamma(\tilde{\tau}-\Gamma)}{(\Gamma(\tilde{\tau}-\Gamma)+1) \tilde{\tau}} \text {. }
$$

The cycle period at maximum cooling power is

$$
\tilde{\tau}_{m}=\Gamma+1 \text {, }
$$

and consequently, the global maximum cooling power is given by

$$
P_{c}=\sqrt{\alpha \mathcal{B}} T_{L} \frac{\Gamma}{(\Gamma+1)}
$$

Producing cooling power according to (3.47) does establish a global upper bound on cooling power for the FTCRC. Though, taking back into account that operation in this maximum cooling power mode is attained only in the limit of zero efficiency, this maximum cooling power mode is referred to as the second degenerate FTCRC operating mode. The zero cooling power quasistatic mode was the first degenerate FTCRC operating mode.

\section{Minimum Power Consumption}

The Lagrangian used to minimize power consumption, using Eq. (3.10a), is given by 


$$
\mathscr{L}=\frac{1}{\tilde{\tau}}\left(\frac{\Delta S T_{h} \Gamma}{\left(\tilde{t}_{h}-\Gamma\right)}-\frac{\Delta S T_{L} \Gamma \tilde{t}_{1}}{\left(\Gamma \tilde{t}_{1}+1\right)}\right)+\mu_{1} \tilde{E}_{1}-\mu_{2}\left(\Gamma-\tilde{t}_{h}\right)
$$

$$
-\lambda\left(\tilde{\tau}-\left(\tilde{t}_{h}+\tilde{t}_{1}\right)\right) \text {. }
$$

Solving $\partial \mathscr{L} / \partial \tilde{E}_{1}=\partial \mathscr{L} / \partial \tilde{t}_{h}=0$ and choosing $\mu_{1}^{*}=\mu_{2}^{*}=0$ and $\lambda^{*}=-1$, the following is obtained:

$$
\epsilon_{h}^{*}=E_{l} \Gamma \sqrt{\theta}+\Gamma+\sqrt{\theta}
$$

Using $\tilde{\tau}=\tilde{E}_{h}+\tilde{t}_{1}$, minimum power consumption occurs when

$$
\tilde{E}_{1}=\frac{\tilde{\tau}-(\Gamma+\sqrt{\theta})}{1+\Gamma \sqrt{\theta}}
$$

and

$$
\tilde{E}_{h}^{*}=\frac{\tilde{\tau} \Gamma \sqrt{\theta}+(\Gamma+\sqrt{\theta})}{1+\Gamma \sqrt{\theta}}
$$

Equations (3.31), (3.49) and (3.50) define the efficiency trajectory of the minimum power operating mode shown in Figure 5 .

The frequency dependent COP is given by

$$
\epsilon(f)=\left(\frac{\theta\left(\Gamma \sqrt{\theta}+\tilde{f}(\Gamma+\sqrt{\theta})\left(\Gamma-\tilde{f}\left(\Gamma^{2}-1\right)\right)\right.}{\Gamma(1-\tilde{f}(\Gamma+\sqrt{\theta}))\left(1-\tilde{f}\left(\Gamma^{2}-1\right)\right)}-1\right)^{-1},
$$

and using (3.52), the first order frequency correction to the quasistatic carnot cop for minimum power consumption is given by 
$\epsilon_{1}^{(P)} \approx \epsilon_{q}\left(1-\frac{\sqrt{\theta}(1+\Gamma \sqrt{\theta})(\Gamma+\sqrt{\theta})}{\Gamma(\theta-1)} f\right)$.

The cycle period at maximum cooling power is

$$
\tilde{\tau}_{m}=\Gamma+\sqrt{\theta}+\frac{1}{\Gamma}(\Gamma(\Gamma+\sqrt{\theta})(1+\Gamma \sqrt{\theta}))^{1 / 2},
$$

and maximum cooling power within this operating mode is given by

$$
P_{c}=\sqrt{\alpha B} T_{L}\left(\left|\left(1+\sqrt{\theta}+\frac{1}{\Gamma} \sqrt{A}\right)(1+\sqrt{B})\right|^{-1},\right.
$$

where

$$
A=\Gamma(\Gamma+\sqrt{\theta})(1+\Gamma \sqrt{\theta})
$$

and

$$
B=\frac{(1+\Gamma \sqrt{\theta})}{\Gamma(\Gamma+\sqrt{\theta})}
$$

Finally, the COP at maximum cooling power while maintaining minimum power consumption is

$$
\epsilon_{m}^{(P)}=\left(\sqrt{\theta}\left(\sqrt{\theta} \frac{1}{\Gamma}(\Gamma(\Gamma+\sqrt{\theta})(1+\Gamma \sqrt{\theta}))^{1 / 2}\right)-1\right)^{-1}
$$

\section{Minimum Irreversible Entropy}

The irreversible entropy production per cycle is given in Eq. (3.11a). The Lagrangian for this optimization is given by

$$
\mathscr{L}=\left(\frac{\Delta S \tilde{t}_{h}}{\left(\tilde{t}_{h}-\Gamma\right)}-\frac{\Delta S \Gamma \tilde{t}_{1}}{\left(\Gamma \tilde{t}_{1}+1\right)}\right)+\mu_{1} \tilde{t}_{1}-\mu_{2}\left(\Gamma-\tilde{t}_{h}\right) \quad \text { (cont.) }
$$




$$
-\lambda\left(\tilde{\tau}-\left(\tilde{E}_{h}+\tilde{E}_{1}\right)\right)
$$

Solving $\partial \mathscr{L} / \partial \tilde{t}_{1}=\partial \mathscr{L} / \partial \tilde{t}_{h}=0$ and setting $\mu_{i}^{*}=\mu_{2}^{*}=0$ with

$$
\begin{aligned}
\lambda^{*}=-1, & \\
\hat{t}_{h}^{*} & =\Gamma \hat{\epsilon}_{1}+\Gamma+1 .
\end{aligned}
$$

Therefore, minimum irreversible entropy production occurs when

$$
E_{1}^{*}=\frac{\tilde{\tau}}{(\Gamma+1)}-1 \text {, }
$$

and

$$
E_{h}^{*}=\frac{\mathfrak{z}}{(\Gamma+1)}+1
$$

The frequency dependent COP is given by

$$
\epsilon(\tilde{f})=\left(\frac{\theta(1+\tilde{E}(\Gamma+1))\left(\Gamma-\tilde{f}\left(\Gamma^{2}-1\right)\right)}{\Gamma(1-\tilde{L}(\Gamma+1))\left(1-\tilde{f}\left(\Gamma^{2}-1\right)\right)}-1\right)^{-1}, \quad \text { (3.60) }
$$

Equations (3.59a) and (3.59b) taken with (3.31) define the minimum irreversible entropy efficiency trajectory shown in Figure 5. To first order in frequency, the cop within the minimum $\Delta S_{I}$ mode is given by

$$
\epsilon_{1}^{\left(\Delta S_{I}\right)} \approx \epsilon_{q}\left(1-\frac{\theta\left(1+\Gamma+\Gamma^{2}-\Gamma^{3}\right)}{\Gamma(\theta-1)} \tilde{f}\right) .
$$

Examining the cooling power within the minimum $\Delta S_{I}$ mode, the period at maximum cooling power is given by 


$$
\tilde{\tau}_{m}=2(\Gamma+1),
$$

and therefore, the maximum cooling power is given by

$$
P_{c}=\frac{\sqrt{\alpha B} T_{L} \Gamma}{2(\Gamma+1)^{2}}
$$

Finally, the cop at maximum cooling power corresponding to minimum irreversible entropy production is given by

$$
\epsilon_{m}^{\left(\Delta S_{I}\right)}=\left(\frac{3 \theta(\Gamma+1)}{\Gamma(3-\Gamma)}-1\right)^{-1}
$$

Two graphical comparisons of the four optimal FTCRC operating modes are made below. First, in Figure 7, curves of $\eta(\tilde{f})=\epsilon(\tilde{f}) / \varepsilon$ 。 are shown as functions of the reduced cooling power $\tilde{P}_{c}(\tilde{f})=P_{c} /\left(T_{L} \sqrt{\alpha B}\right)$ for the maximum efficiency, minimum irreversible entropy production, and minimum power consumption operating modes. It is evident from examining these curves that achieving maximum cooling power requires a significant reduction in cycling efficiency. In the maximum efficiency operating mode, for example, achieving the last 25\% increase towards maximum cooling power results in roughly a $50 \%$ decrease in cycling efficiency. It is also seen that among the four operating modes considered in this chapter that the maximum efficiency cycling mode not only achieves the highest COP at a given cycling frequency, but also produces the greatest cooling power for a given nonzero efficiency. The degenerate maximum cooling power mode does 


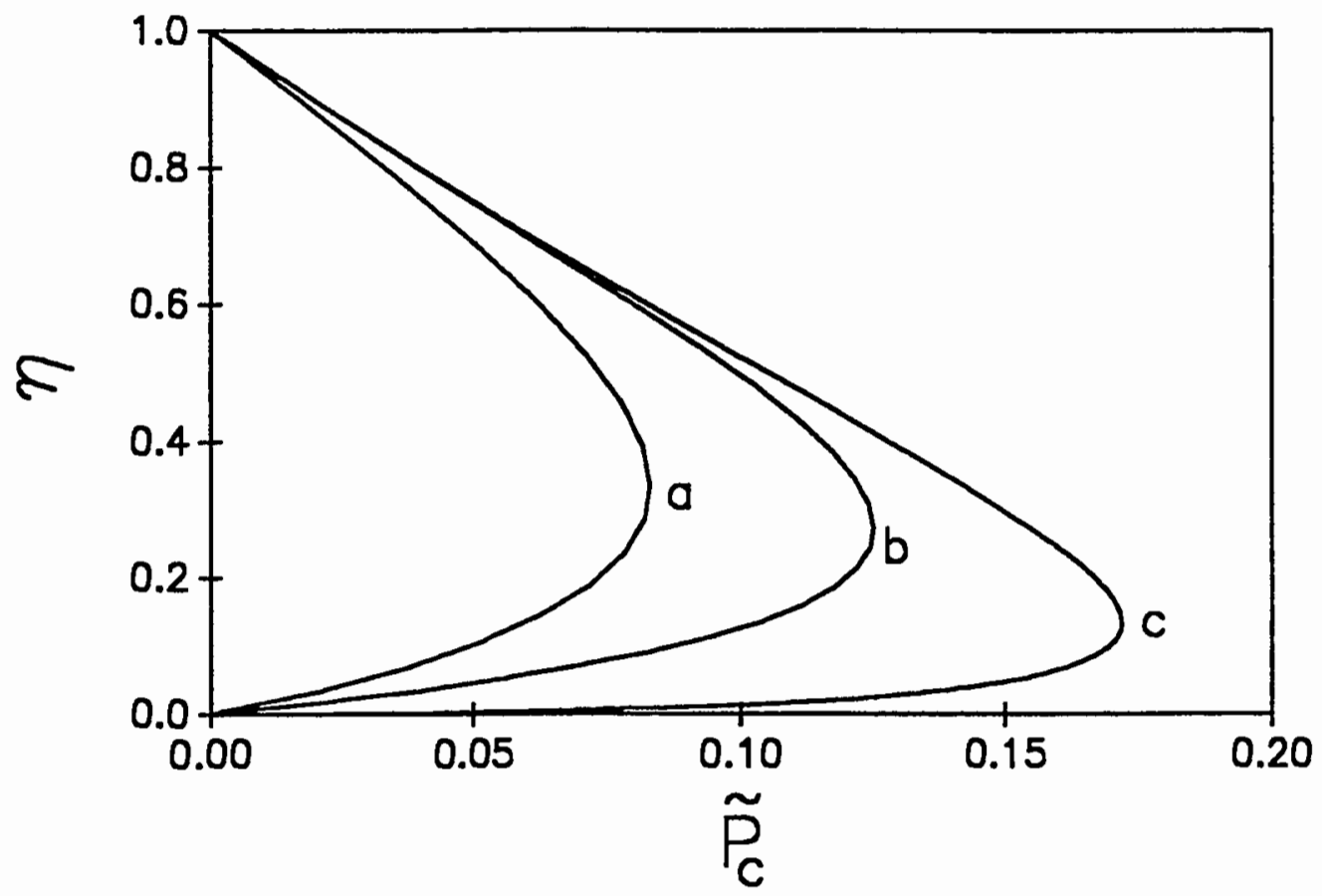

Figure 7. Reduced coefficient of performance, $\eta$, vs reduced cooling power, $\tilde{P}_{c}$, for three optimal refrigeration cycling modes. Curves $a, b$, and $c$ represent the minimum power consumption, minimum irreversible entropy production, and maximum efficiency FTCRC operating modes, respectively, for $\theta=3$ and $\Gamma=1$. 
generate more cooling power than the maximum efficiency mode, as shown in Figure 6 , but at the thermodynamic expense of operating at the zero efficiency limit and therefore, is physically uninteresting. In the zero cooling power limit, all FTCRC operating modes converge to the quasistatic Carnot mode represented in Figure 7 by the point $\left(\eta, \tilde{P}_{c}\right)=(1.0,0.0)$.

Secondly, the four optimal cycling modes are compared in Figure 8 within a three dimensional surface plot defined by the reduced cycling frequency $\tilde{f}$, cooling power $\tilde{P}_{c}$, and efficiency $\eta$. This three dimensional surface is composed of contours of constant cycling frequency in the same way as the efficiency space discussed earlier. Point $A$, where $\left(\tilde{f}, \tilde{P}_{c}, \eta\right)=(0.0,0.0,1.0)$, corresponds to the degenerate zero cooling power quasistatic operating mode, and $\left(\tilde{f}, \tilde{P}_{c}, \eta\right)=(1.0,0.0,0.0)$ corresponds to the infinite power consumption mode at zero efficiency and cooling power. The trajectories along which the points $B, C, D$, and E lie represent the minimum power consumption, minimum irreversible entropy production, maximum efficiency, and maximum cooling power operating modes, respectively, where the labeled points indicate operation at maximum cooling power. In addition, it is clear from Figure 8 that FTCRC operating modes exist that have not been discussed in this chapter. For example, an operating mode corresponding to a 


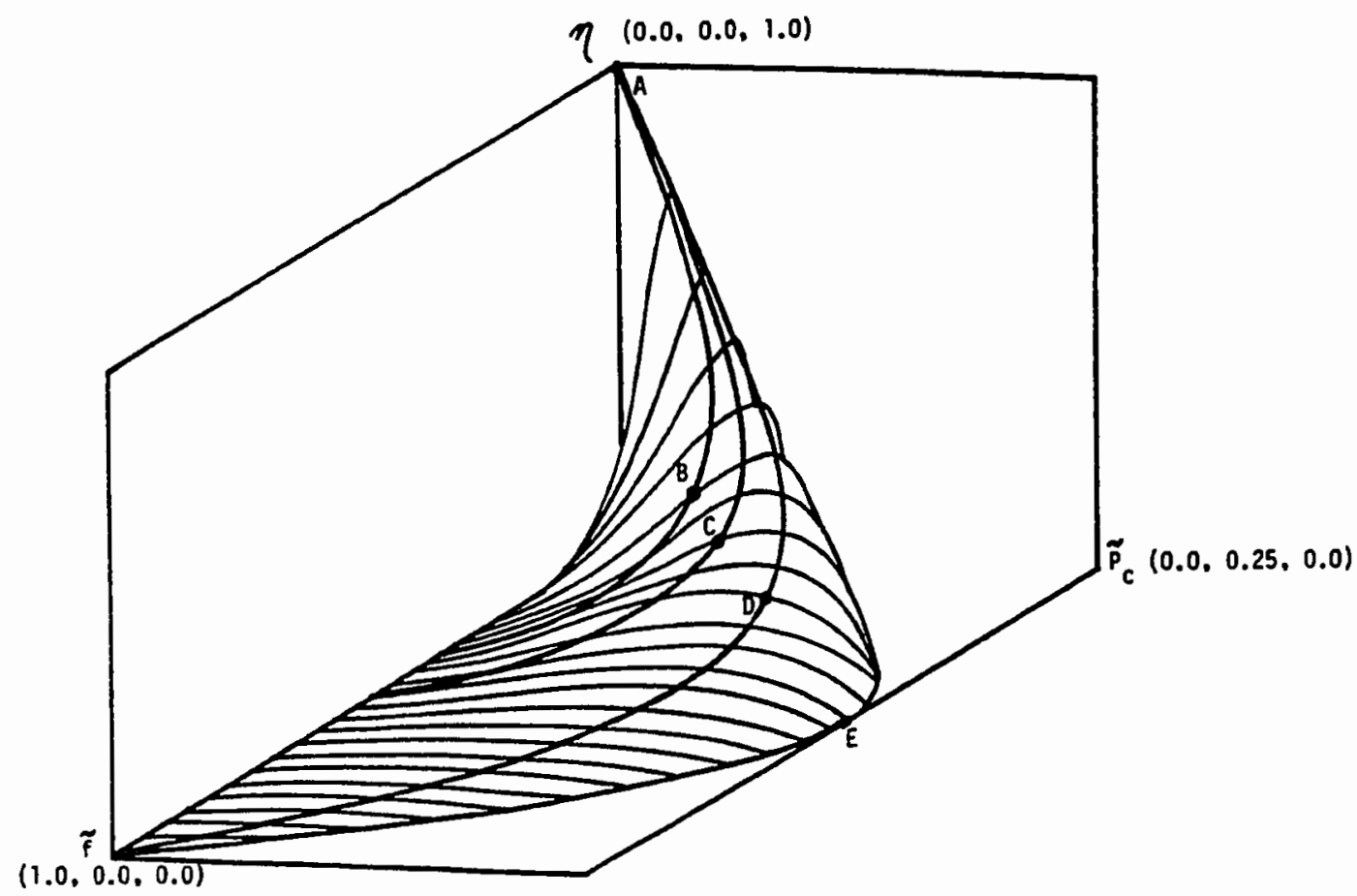

Figure 8. Three dimensional surface of reduced cycling frequency, $\tilde{E}$, cooling power, $\tilde{P}_{c}$, and coefficient of performance, $\eta$. The surface plot is composed of contours of constant cycling time, and $\boldsymbol{\theta}=3$ and $\Gamma=1$. Point $A$ indicates the zero cooling power maximum Carnot efficiency quasistatic operating mode. Points B, C, D, and E are the coefficients of performance at maximum cooling power within the minimum power consumption, minimum irreversible entropy production, maximum efficiency, and maximum cooling power operating modes, respectively. 
trajectory in Figure 8 falling between points $D$ and $E$ generates more cooling power than the maximum efficiency mode but less cooling power than the maximum cooling power mode. These additional FTCRC operating modes could be obtained by appropriately optimizing the FTCRC to fulfill a refrigeration performance objective other than one of the four objectives discussed in this chapter.

\section{SUMMARY AND CONCLUSION}

The problem of obtaining optimal operating modes and these modes respective efficiencies for a simple finite time refrigeration cycle (FTRC) model has been examined. In contrast to the traditional quasistatic carnot refrigeration cycle, FTRC's operate irreversibly over a continuous range of cycling times and produce finite cooling power. Specifically, the FTCRC model has been optimized to obtain the four distinct operating modes that maximize thermal efficiency and cooling power, and minimize power consumption and irreversible entropy production. It is found that regardless of the optimization objective, that for the three nondegenerate optimum cycling configurations, the FTCRC should always devote more cycle time to absorbing heat from the low temperature thermal reservoir than to exhausting heat to the high temperature reservoir. This latter strategy for heat exchange may be useful when considering a time dependent mechanisms which drives a 
refrigerant through an irreversible refrigeration process. Examples of these mechanisms are the motion of a piston in a reciprocating refrigeration cycle and the manner in which magnetic material is cycled through an external magnetic field in a magnetic refrigeration cycle. In addition, it has been shown that for all four objective functions considered here that optimal operating modes are attained when heat transfer occurs isothermally and when the adiabatic branches are traversed instantaneously. Thus, the optimal cycle is a finite time Carnot refrigeration cycle (FTCRC) which is the refrigeration cycle analog to the finite time Carnot engine studied by Ahlborn (2), Salamon (4), Rubin (5), and others $(8,9)$.

Refrigeration cycle efficiency is conveniently represented within a three dimensional efficiency space. Surfaces within the efficiency space are defined in terms of the reduced FTCRC efficiency and two optimal control variables which in the present case are $\tilde{t}_{1}$ and $\tilde{t}_{h}$ the dimensionless times devoted to low and high temperature heat transfer, respectively. Perhaps the most useful aspect of constructing these three dimensional surfaces is the global insight one is able to gain into the efficiency properties of an FTCRC. By examining efficiency space surfaces, differences between the four FTCRC optimal operating modes that are not otherwise obvious through studying mathematical equations become visually apparent. For example, one can 
gain instant insight into the relative cycling efficiency properties of the maximum efficiency and cooling power, and minimum power consumption and minimum irreversible entropy production modes by the location within the efficiency space of these mode's efficiency trajectories.

Simple analytic expressions have been obtained for the following two efficiencies in each of the four optimum cycling modes: 1) the first order correction in cycling frequency to the quasistatic carnot CoP for nonzero cycling times and 2) the coefficients of performance at maximum cooling power. These latter coefficients of performance may be more useful than the quasistatic carnot coefficient of performance as estimates of the bound on thermal efficiency for certain refrigeration cycles that maximize efficiency while producing cooling power.

Considering further the efficiency and cooling power properties of the four optimal modes that are obtained in this chapter, the maxima in cooling power and the efficiencies evaluated at these cooling power maxima are ordered according to the following:

$$
0=P_{c}^{0}<P_{c}^{(P)}<P_{C}^{\left(\Delta S_{I}\right)}<P_{c}^{(e)}<P_{c}^{\left(P_{c}\right)}
$$

and

$$
0=\epsilon_{m}^{\left(P_{c}\right)}<\epsilon_{m}^{(e)}<\epsilon_{m}^{\left(\Delta s_{p}\right)}<\epsilon_{m}^{(P)}<\epsilon_{o},
$$

where the superscripts in Eq. (3.67), for example, indicate the maximum cooling power, maximum efficiency, minimum 
irreversible entropy production, and minimum power consumption operating modes, respectively. The symbols $P_{c}^{0}$ and $\epsilon_{o}$ represent the cooling power and efficiency of the degenerate quasistatic operating mode.

of most interest to the present work on obtaining new bounds on thermal efficiency of refrigeration cycles are the efficiencies at maximum cooling power comprising Eq. (3.67). Relative to the global maximum quasistatic carnot efficiency, the efficiencies in (3.67) constitute a set of successively lowered bounds on the thermal efficiency of certain carnot like refrigeration cycles operating at maximum cooling power. Taking into account that the degenerate maximum cooling power mode operates uselessly at zero efficiency, $\boldsymbol{\epsilon}_{m}^{(e)}$ of the maximum efficiency mode constitutes a least upper bound on the efficiency of certain Carnot like refrigeration cycles operating at maximum efficiency and cooling power. In addition, in the special limiting case of equal heat transfer coefficients at the high and low temperatures, $\epsilon_{m}^{(e)}$ reduces to $\varepsilon_{m}$ a general limiting efficiency at maximum cooling power obtained in Chapter II through a much less general analysis. As a final note, these new bounds on thermal efficiency must be applied with caution. Even though these bounding efficiencies retain much of the inherent simplicity 
which made the quasistatic carnot COP so generally useful, to attempt to extend these results beyond carefully examined cases becomes a difficult thing to do. Clearly, as seen in Figures 7 and 8 , the finite time coefficients of performance at maximum cooling power are not fundamental bounds on thermal efficiency in the same sense as the carnot cop. It is a simple matter to exceed the efficiencies at maximum cooling power and to operate an FTCRC at an efficiency arbitrarily close to the quasistatic carnot maximum. Therefore, it is important to stress that the new bounds on thermal efficiency obtained above may apply only to refrigeration cycles operating at maximum cooling power and in one of the four optimized refrigeration cycling modes discussed in this chapter. 
CHAPTER IV

\author{
MAXIMUM EFFICIENCY OF A FINITE TIME \\ REFRIGERATION CYCLE WITH \\ NON-IDEAL HEAT SWITCHES
}

INTRODUCTION

The problem of non-ideal heat switches linking the working fluid of a finite time refrigeration cycle to the external thermal reservoirs is next examined. A significant source of irreversibility in the form of heat leaks may exist due to the non-ideal switching $(14,15)$. If a refrigeration cycle is carnot like, when traversing the low temperature isothermal branch heat is absorbed in the usual way from the low temperature reservoir into the working fluid, but due to non-ideal thermal switching, heat will also leak into the working fluid from the high temperature reservoir. Heat will leak back into the low temperature reservoir while operating the refrigeration cycle along the high temperature isothermal branch. It is important, therefore, to understand the effects of non-ideal switching on cycle efficiency.

An extended finite time refrigeration cycle model incorporating non-ideal heat switches is used to study this 
problem. The basis of the refrigeration cycle model is still the idealized endoreversible cycle (5) with Newton's law heat fluxes coupling two external isothermal reservoirs to the working fluid. The refrigeration cycle operates cyclicly over two ideal reversible adiabatic branches over which no heat leaks occur and two initially arbitrary heat transfer branches. Euler-Lagrange variational methods are used to show that optimum heat transfer occurs isothermally even with non-ideal heat switches, and therefore, the cycle considered here is a finite time carnot refrigeration cycle (FTCRC) .

To examine the efficiency of the FTCRC with non-ideal heat switches, a three dimensional efficiency space is defined. Paths within the efficiency space that correspond to possible refrigeration cycle modes of operation are referred to as efficiency trajectories. The standard coefficient of performance (COP) is used as an efficiency measure and because the current problem is sufficiently more complicated mathematically than those discussed in chapters II and III, numerical optimization methods are used to show that there exists a globally optimum efficiency trajectory within the efficiency space. This optimum trajectory defines the FTCRC operating mode that maximizes efficiency. The performance of finite time carnot refrigeration cycles with ideal and non-ideal heat switches are compared. Relative to an FTCRC with ideal switches, non-ideal heat 
switches significantly alter the efficiency and cooling power characteristics of finite time carnot refrigeration cycles. Most notably, an FTCRC with non-ideal switches has certain operating regimes in which cooling power and efficiency are negative. In addition, it is found that there exist two distinct optimum cycling conditions for a cycle with non-ideal heat switches: 1) operation at the global maximum in efficiency, and 2) operation at the frequency of maximum cooling power. The cop evaluated at maximum cooling power and global maximum COP may provide improved bounds on the efficiency of real irreversible refrigeration cycles with non-ideal heat switches that maximize efficiency while producing cooling power.

\section{REFRIGERATION CYCLE MODEL}

The performance of a refrigeration cycle operating at nonzero cycling frequencies that is in thermal contact with two isothermal heat reservoirs through non-ideal heat switches is examined. This latter refrigeration cycle with non-ideal heat switches is shown in Figures 9 and 10 (Figure 10 is adapted from Hakuraku (14)). Two of the four cycle branches are defined to be reversible adiabats, and the other two are the high and low temperature branches along which heat is exchanged with the external reservoirs and whose temperatures are initially arbitrary functions of time. Since the heat switches connecting the working fluid 


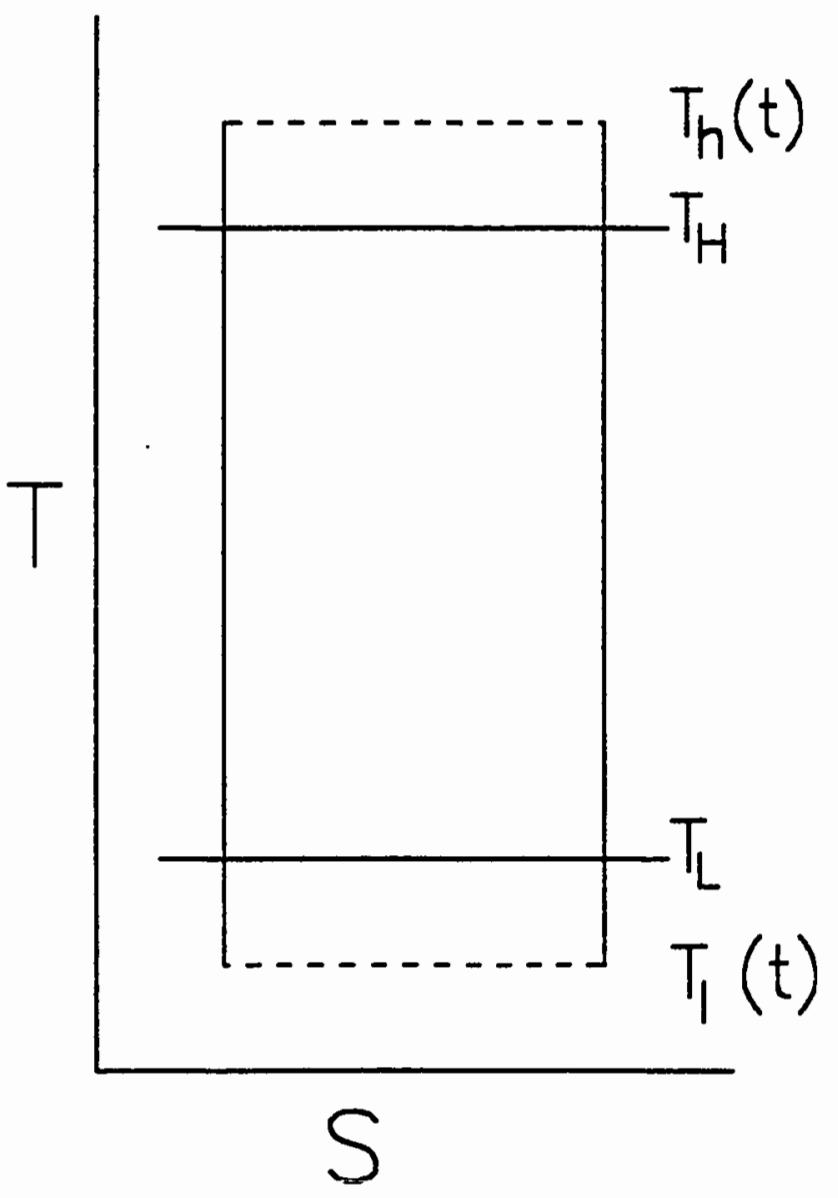

Figure 9. Temperature-entropy state space diagram of a finite time refrigeration cycle with nonideal heat switches. The temperatures $T_{H}$ and $T_{L}$ are the high and low temperatures of the heat reservoirs, respectively. $T_{h}$ and $T_{1}$ are the high and low temperatures of the working fluid during heat transfer, respectively. 
High Temp. Reservoir $\left(T_{H}\right)$

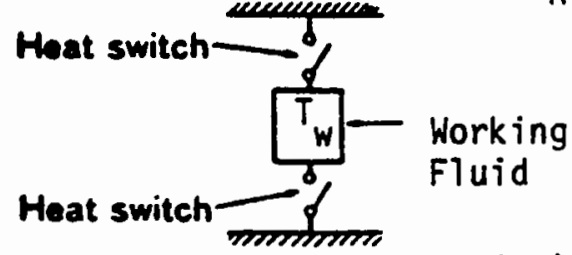

Low Temp. Reservoir $\left(T_{L}\right)$

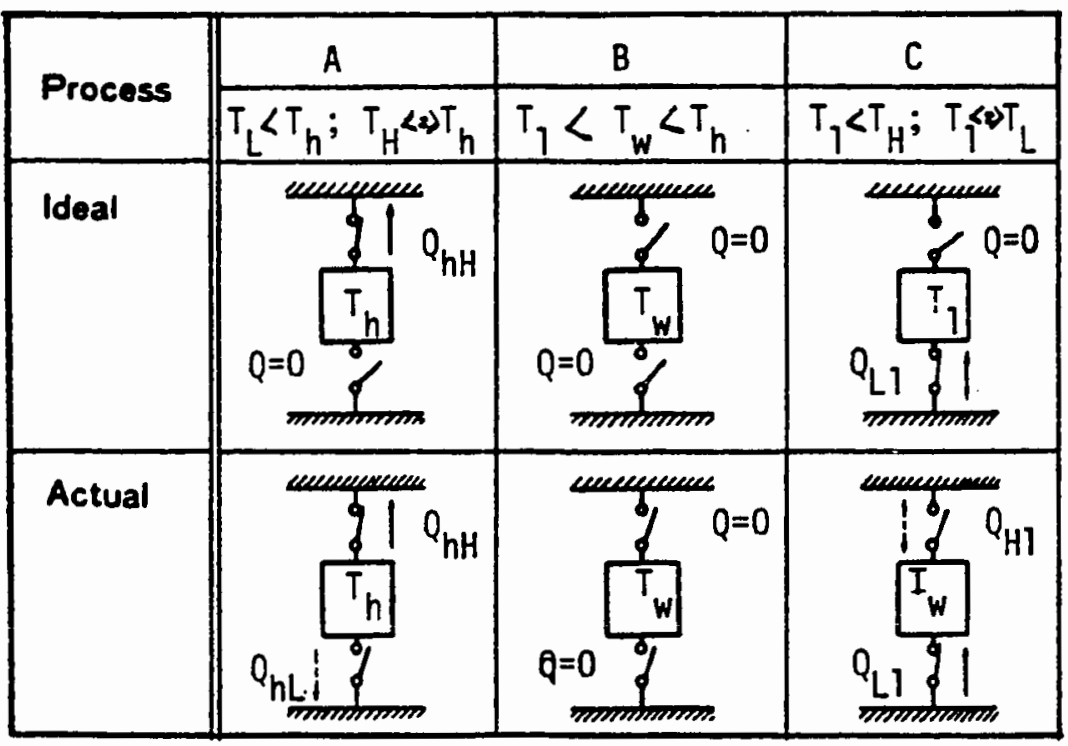

Figure 10. Schematic of a finite time refrigeration cycle with non-ideal heat switches. In frame $A$ heat $Q_{h H}$ is exhausted from the working fluid at temperature $T_{h}$ into the heat reservoir at $T_{H}$. Simultaneously, heat $Q_{h I}$ is leaked into the low temperature reservoir at $T_{L}$. Frame $B$ shows that zero heat is transferred along both ideal adiabatic branches. In frame $c$ heat $Q_{L 1}$ is absorbed from the low temperature reservoir at $T_{L}$ into the working fluid at temperature $T_{1}$. At the same time $Q_{H 1}$ is leaked into the working fluid from the high temperature reservoir at $T_{H}$. 
to the external heat reservoirs do not function ideally, a source of irreversibility in the form of heat leaks exists. When traversing the low temperature heat transfer branch, heat is absorbed from the low temperature thermal reservoir into the working fluid, but due to non-ideal thermal switching, heat will also leak into the working fluid from the high temperature thermal reservoir. In a similar way, heat will leak back into the low temperature reservoir while operating the refrigeration cycle along the high temperature heat transfer branch. The combined system consisting of the refrigeration cycle and the two external thermal reservoirs is isolated from all other systems.

The following set of assumptions define the finite time endoreversible refrigeration cycle with non-ideal heat switches:

1. The working fluid undergoes only reversible transformations throughout operation of the refrigeration cycle. The sum of the entropy changes in the working fluid over one cycle add to zero, i.e.,

$$
\oint d S=0 .
$$

2. Heat fluxes between the working fluid of the refrigeration cycle and external reservoirs are assumed to be governed by Newton's law of cooling which is given by 


$$
\dot{Q}=\frac{d Q}{d t}=h\left(T_{R}-T_{w}(t)\right)
$$

where $T_{R}$ represents the high and low temperature of the external thermal reservoirs and can be either $T_{H}$ or $T_{L}$, respectively. The constant $\mathrm{h}$ represents the heat transfer coefficient, where $h=B$ or $h=\alpha(\alpha, B>0)$ along the high and low temperature heat transfer branches, respectively. Also, $T_{w}(t)$ represents the temperature of the working fluid at time $t$ and can be either $T_{h}(t)$ or $T_{1}(t)$, where $T_{h}(t)$ and $T_{1}(t)$ are the high and low temperatures of the working fluid during heat transfer, respectively. Because $T_{1}(t)$ may be less than, equal to, or greater than $T_{L}, \dot{Q}=\dot{Q}_{L 1} \Leftrightarrow 0$ for heat flux from the external thermal reservoir at the temperature $T_{L}$ into the working fluid at $T_{1}(t)$ along the low temperature branch. Because $T_{h}(t)$ may be greater than, equal to, or less than $T_{H}, \dot{Q}=\dot{Q}_{h H} \Leftrightarrow 0$ for heat flux from the working fluid at the temperature $T_{h}(t)$ into the high temperature thermal reservoir at $T_{H}$ along the high branch.

3. Heat flux through non-ideal thermal switches between the working fluid of the refrigeration cycle and external reservoirs are assumed to be governed by Newton's law 
of cooling and are given by

$$
\dot{Q}^{\prime}=\frac{d Q^{\prime}}{d t}=k\left(T_{R}^{\prime}-T_{W}(t)\right)
$$

where $T_{R}^{\prime}$ represents the temperature of the external reservoirs contributing to the heat leak and can be either $T_{H}$ or $T_{L}$. The constant $\mathrm{k}$ represents the heat transfer coefficient governing the heat leak, where $\mathrm{k}=k_{h}$ or $\mathrm{k}=k_{1}$ $\left(k_{h}, k_{1}>0\right)$ along the high and low temperature heat transfer branches, respectively. Also, $T_{w}(t)$ represents the temperature of the working fluid at time $t$ and can be either $T_{h}(t)$ or $T_{1}(t)$ which are the high and low temperatures of the working fluid while heat leaks through the thermal switches, respectively. Because $T_{I}(t)<T_{H}$, $\dot{Q}=\dot{Q}_{H 1}>0$ for heat leak from the high temperature thermal reservoir into the working fluid at temperature $T_{1}(t)$ while the refrigeration cycle works to extract heat from the low temperature reservoir. Similarly, because $T_{L}<T_{h}(t)$, $\dot{d}=\dot{Q}_{h L}<0$ for heat leak from the working fluid at the temperature $T_{b}(t)$ into the low temperature thermal reservoir at $T_{L}$ while the refrigeration cycle traverses the high temperature branch. 
4. The combined heat transferred over the high and low temperature branches due to imperfect thermal switching and normal heat transfer is constrained to produce a constant entropy change in the working fluid which is given by

$$
\Delta S=\int_{0}^{t}\left(\frac{\left|\dot{Q}+\dot{Q}^{\prime}\right|}{T_{w}(t)}\right) d t=\text { const. }
$$

where $t_{w}=t_{h}$ and $t_{w}=t_{l}$ are the times taken to traverse the high and low temperature heat transfer branches, respectively. In addition, $-\Delta S=\Delta S_{h}$ and $\Delta S=\Delta S_{1}$ are the entropy changes in the working fluid during high and low temperature heat transfer, respectively. The entropy change over a refrigeration cycle is $\Delta S_{h}+\Delta S_{1}=0$ which satisfies assumption 1. As will be shown below, using Eq. (4.4) makes it possible to obtain analytic expressions for $T_{h}(t)$ and $T_{l}(t)$ in terms of $t_{h}$ and $t_{l}$ without resorting to a specific equation of state for the working fluid.

Finally, the total heat absorbed and rejected by the working fluid along either the high or low temperature branches, taking into account the heat leak and using Eqs. $(4.2)$ and $(4.3)$, is given by

$$
Q=\int_{0}^{t_{w}}\left(\dot{Q}_{w}+\dot{Q}_{w}\right) d t
$$

where

$$
Q_{w}=\int_{0}^{t_{w}} h\left(T_{R}-T_{w}(t)\right) d t,
$$


and

$$
Q_{w}^{\prime}=\int_{0}^{t_{w}} k\left(T_{R}^{\prime}-T_{w}(t)\right) d t
$$

Heat transfer from the working fluid at the temperature $T_{h}$ is given by $Q=Q_{h}=Q_{h H}+Q_{h L}$, and heat transfer from the working fluid at the temperature $T_{1}$ is given by $Q=Q_{1}=Q_{1 L}+Q_{H 1}$. Equations (4.5) - (4.7) will be used below to obtained the optimum working fluid temperature as a function of time that maximizes efficiency.

Throughout this chapter, the convention will be used that lower case subscripts represent the working fluid and upper case subscripts refer to the external reservoirs.

\section{OPTIMIZATION OF CYCLE BRANCHES}

The temperature of the working fluid as a function of time throughout cycle operation that will maximize the efficiency is obtained. First, the efficiency is shown to be maximized when the magnitude of the heat rejected to the high temperature reservoir is minimized and when the heat absorbed from the low temperature reservoir is maximized. Both heats are extremized when heat transfer occurs isothermally. The adiabatic branches are assumed to be traversed in a time proportional to the times allotted to the heat transfer branches. The time path the temperature of the working fluid follows to maximize the efficiency of 
an FTCRC with non-ideal heat switches is such that the working fluid temperature remains isothermal during heat transfer and changes between $T_{h}$ and $T_{1}$ along the adiabatic branches.

Objective Function

The efficiency can be expressed in terms of $Q_{H}\left(t_{h}, t_{1}\right)=Q_{h H}\left(t_{h}\right)+Q_{H I}\left(t_{1}\right)$ the net heat exhausted to the high temperature thermal reservoir, $Q_{L}\left(t_{h}, t_{1}\right)=Q_{L I}\left(t_{1}\right)+Q_{h L}\left(t_{h}\right)$ the net heat absorbed from the low temperature thermal reservoir, and $\tau=t_{h}+t_{1}+t_{A}$ the cycle period, where $t_{A}$ is the time devoted to the adiabatic branches.

The coefficient of performance (COP) is defined (15) as

$$
\epsilon\left(t_{h}, t_{1}\right)=\frac{e_{L}\left(t_{h}, t_{1}\right)}{-W_{n e t}\left(t_{h}, t_{1}\right)},
$$

where $W_{n \theta t}\left(t_{h}, t_{1}\right)=Q_{H}\left(t_{h}, t_{1}\right)+Q_{L}\left(t_{h}, t_{1}\right)$, or

$$
\boldsymbol{\varepsilon}\left(t_{1}, t_{h}\right)=\left(-\left(\frac{Q_{H}\left(t_{1}, t_{h}\right)}{Q_{L}\left(t_{1}, t_{h}\right)}\right)-1\right)^{-1} .
$$

The changes in efficiency due to changes in $Q_{H}\left(t_{h}, t_{1}\right)$ and $Q_{L}\left(t_{h}, t_{1}\right)$, respectively, are given by the following:

$$
\frac{\partial \varepsilon}{\partial Q_{H}}=\frac{1}{Q_{L}}\left(-\left(\frac{Q_{H}}{Q_{L}}\right)+1\right)^{-2}>0,
$$




$$
\begin{aligned}
& \frac{\partial^{2} \epsilon}{\partial Q_{H}^{2}}=\frac{-2}{Q_{L}^{2}}\left(-\left(\frac{Q_{H}}{Q_{L}}\right)-1\right)^{-3}<0, \\
& \frac{\partial \epsilon}{\partial Q_{L}}=\frac{-Q_{H}}{Q_{L}^{2}}\left(-\left(\frac{Q_{H}}{Q_{L}}\right)-1\right)^{-2}>0,
\end{aligned}
$$

and

$$
\frac{\partial^{2} \epsilon}{\partial Q_{L}^{2}}=\frac{2 Q_{H}}{Q_{L}^{3}}\left(-\left(\frac{Q_{H}}{Q_{L}}\right)-1\right)^{-2}\left(1-\frac{1}{1+\frac{Q_{L}}{Q_{H}}}\right)<0 .
$$

From examining Eqs. (4.10a), (4.10b), (4.11a), and (4.11b), it is seen that efficiency is maximized when the magnitude of the heat exhausted to the high temperature reservoir is minimized and the heat absorbed from the low temperature reservoir is maximized.

\section{Optimizing Heat Transfer}

The problem now is to obtain the temperature of the working fluid as a function of time along heat transfer and adiabatic cycle branches that extremizes both $Q_{H}\left(t_{h}, t_{1}\right)$ and $Q_{L}\left(t_{h}, t_{1}\right)$

Heat Transfer Branches. It is shown in Appendix B that optimizing $Q_{h}\left(t_{h}, t_{1}\right)$ and $Q_{1}\left(t_{h}, t_{1}\right)$, the net heats transferred into and out of the working fluid, is equivalent to and mathematically much less complicated than optimizing 
$Q_{H}\left(t_{h}, t_{1}\right)$ and $Q_{L}\left(t_{h}, t_{1}\right)$ the net heats rejected to and absorbed from the external thermal reservoirs, respectively. Therefore, for convenience and mathematical clarity, $Q_{h}\left(t_{h}, t_{1}\right)$ and $Q_{1}\left(t_{h}, t_{1}\right)$ are extremized to obtain the optimum temperature of the working fluid during high and low temperature heat transfer, respectively.

Using the classical Euler-Lagrange method and Eqs. (4.4) and (4.5), the Lagrangian for the present optimization is given by

$$
\mathscr{L}=\dot{Q}_{w}-\lambda \frac{\dot{Q}_{w}}{T_{w}}
$$

and

$$
\mathscr{L}=\left(h\left(T_{R}-T_{w}(t)\right)+k\left(T_{R}^{\prime}-T_{w}(t)\right)\right)\left(1-\frac{\lambda}{T_{w}(t)}\right)
$$

where $\lambda$ is a Lagrange multiplier. The optimal $T_{w}(t)$ obtained from the simplified Euler-Lagrange equation $\partial \mathscr{L} / \partial T_{w}(t)=0$, is

$$
T_{w}(t)=\sqrt{\frac{\lambda\left(h T_{R}+k T_{R}^{\prime}\right)}{(h+k)}}=\text { const. }>0 .
$$

The Lagrange multiplier is

$$
\lambda=\frac{T_{w}^{2}(h+k)}{\left(h T_{R}+k T_{R}^{\prime}\right)}>0,
$$


and from the Legendre condition,

$$
\frac{\partial^{2} \mathscr{L}}{\partial T_{w}^{2}}=\frac{-2 \lambda\left(h T_{R}+k T_{R}^{\prime}\right)}{T_{w}^{3}}<0 .
$$

The free endpoint conditions (23)

$$
\left.\frac{\partial \mathscr{L}}{\partial \dot{T}_{w}}\right|_{t=0}=\left.\frac{\partial \mathscr{L}}{\partial \dot{T}_{w}}\right|_{t=t_{v}}=0
$$

are automatically satisfied. It follows from Eqs. (4.14) (4.17) that the optimal way to minimize heat leak through thermal switches and transfer heat between a thermal reservoir at a fixed temperature and an endoreversible working fluid undergoing an entropy change $\Delta S_{w}$, in time $t_{w}$, is isothermally. In addition, as with the FTCRC of Chapter III, optimal heat transfer branches should be made up of only one continuous isotherm (3). Therefore, using Eq. (4.4), the temperature of the working fluid is given by

$$
T_{w}=\frac{h T_{R}+k T_{R}^{\prime}}{\left(h+k+\frac{\Delta S_{w}}{t_{w}}\right)}
$$

Finally, as is Chapter III, because the freedom exists to connect the isothermal heat transfer branches with discontinuous adiabatic transitions, it is not a concern that the solution to the present Euler-Lagrange problem 
will, in general, not fall at the cemperatures $T_{w}(0)$ and $T_{w}\left(t_{w}\right)$ at the times $t=0$ and $t=t_{w}$, respectively.

Using Eqs. (4.6) and (4.18), heat transfer while traversing the high and low temperature isotherms, respectively, not due to heat leak, is given by

$$
Q_{L 1}\left(\tilde{t}_{1}\right)=\frac{\Delta S T_{L} \Gamma \tilde{E}_{1}\left(1-\Gamma \tilde{E}_{1} \tilde{k}_{1}(\theta-1)\right)}{\left(\Gamma \tilde{t}_{1}\left(1+\tilde{k}_{1}\right)+1\right)}
$$

and

$$
\ell_{h H}\left(\tilde{t}_{h}\right)=\frac{-\Delta S T_{h} \tilde{t}_{h}\left(\Gamma-\tilde{t}_{h} \tilde{k}_{h}(1-1 / \theta)\right)}{\Gamma\left(\tilde{t}_{h}\left(1+\tilde{k}_{h}\right)-\Gamma\right)}
$$

where $\Delta S_{w}=\Delta S_{1}=-\Delta S_{h}=\Delta S$ has been used. In addition, the following dimensionless variables have been introduced:

$$
\begin{aligned}
& \Gamma=\sqrt{\frac{\alpha}{B}}, \\
& E_{1}=t_{1} \frac{\sqrt{\alpha B}}{\Delta S}, \\
& \tilde{E}_{h}=t_{h} \frac{\sqrt{\alpha B}}{\Delta S}, \\
& \tilde{k}_{1}=\frac{k_{1}}{\alpha}, \\
& \tilde{k}_{h}=\frac{k_{h}}{B},
\end{aligned}
$$


and

$$
\theta=\frac{T_{H}}{T_{L}}
$$

The heat leak coefficients $\tilde{k}_{h}$ and $\tilde{k}_{1}$ in (4.24) and (4.25) determine how normal heat transfer is affected due to the presence of the non-ideal thermal switches. When numerical results are required, the cycle parameters $\tilde{k}_{1}=0.05$, $\tilde{k}_{h}=0.01, \Gamma=1.0$, and $\theta=3.0$ are used. These values for the heat-leak coefficients are representative of the relative heat transfer rates for condensation and boiling vs conductive heat transfer in a refrigeration cycle (14). setting $\tilde{k}_{h}=\tilde{k}_{1}=0$ in Eqs. (4.19) and $(4.20)$, Eqs. (3.19) and (3.20) for heat transfer through ideal thermal switches are recovered.

Heat traisfer due to heat leak through the non-ideal thermal switches is given by

$$
\ell_{h L}\left(\tilde{E}_{h}\right)=\frac{-\Delta S T_{h} \tilde{k}_{h} \tilde{E}_{h}\left(\Gamma+\tilde{E}_{h} \tilde{k}_{h}(\theta-1)\right)}{\Gamma\left(\tilde{E}_{h}\left(1+\tilde{k}_{h}\right)-\Gamma\right)}
$$

and

$$
Q_{H I}\left(\tilde{E}_{1}\right)=\frac{\Delta S T_{L} \Gamma \tilde{k}_{1} \tilde{E}_{1}\left(\theta+\Gamma \tilde{E}_{1}(\theta-1)\right)}{\left(\Gamma \tilde{E}_{1}\left(1+\tilde{k}_{1}\right)+1\right)}
$$

Heat leak given by $(4.27)$ and $(4.28)$ reduces to zero in the 
limit of $\tilde{k}_{h}=\tilde{k}_{1}=0$ for ideal heat switch operation.

Adiabatic Branches. The standard assumption is made for this class of finite time cycles that time spent along the two adiabatic branches is on a time scale consistent with minimizing heat leaks, and is negligible compared to the time taken to traverse the two heat transfer branches $(3,5,17)$. It is also assumed that time spent along the two adiabats is proportional to the time spent along the heat transfer branches. Therefore, $t_{A}=k_{A}\left(t_{h}+t_{1}\right)$, where $t_{A}$ is the time spent along the adiabats and $k_{A}$ is a proportionality constant. The total cycling time $\tau^{\prime}=t_{h}+t_{1}+t_{A}$ then becomes $\tau_{A}^{\prime}=k_{A}^{\prime}\left(t_{h}+t_{1}\right)$, where $k_{A}^{\prime}=k_{A}+1 . \quad$ A rescaled cycle period is defined as $\tau=\left(\tau_{A}^{\prime} / k_{A}^{\prime}\right)=t_{h}+t_{1}$. This approach has been used in studies of finite time heat engines $(2,3,5,17,18)$. since some nonzero time must be spent along the adiabatic branches, the idea of spending negligible time along the adiabats is interpreted to mean that the time scale for traversing the adiabats is small compared to the time scale for heat transfer along the isotherms but is large compared to the time scale of the internal relaxations of the working fluid. This is consistent with the physical interpretation given here and elsewhere as to the nature of endoreversible cycles (5). 
The optimum time path the temperature of the working fluid follows to extremize heat transfer and maximize the efficiency of an FTCRC with non-ideal thermal switches is such that the working fluid temperature remains isothermal during heat transfer and switches, in the sense described above, between $T_{h}$ and $T_{l}$ along the two adiabatic branches.

\section{MAXIMUM EFFICIENCY}

To obtain the optimal FTCRC operating mode that maximizes efficiency, the efficiency function is maximized by numerically optimizing the relative distribution of cycling time devoted to high and low temperature heat transfer. This latter optimization yields the high and low temperatures of the working fluid during heat transfer that maximize cycling efficiency. Using optimal numerical solutions, the following two efficiencies are obtained: 1) the global maximum coefficient of performance and 2) the coefficient of performance at maximum cooling power.

Using Eqs. $(4.19),(4.20),(4.27)$, and $(4.28)$, the COP of Eq. (4.9) is given by

$$
\epsilon\left(\tilde{E}_{h}, \tilde{E}_{1}\right)=\left(\frac{A-B}{C-D}-1\right)^{-1},
$$

where

$$
A=\frac{T_{L} \Gamma \tilde{E}_{1}\left(1-\Gamma \tilde{E}_{1} \tilde{k}_{1}(\theta-1)\right)}{\Gamma \tilde{E}_{1}\left(1+\tilde{k}_{1}\right)+1},
$$




$$
\begin{aligned}
& B=\frac{T_{L} \Gamma \tilde{k}_{1} \tilde{t}_{1}\left(\theta+\Gamma \tilde{t}_{1}(\theta-1)\right)}{\Gamma \tilde{t}_{1}\left(1+\tilde{k}_{1}\right)+1}, \\
& C=-\left(\frac{T_{h} \tilde{k}_{h} \tilde{t}_{h}\left(\Gamma+\tilde{t}_{h} \tilde{k}_{h}(\theta-1)\right)}{\Gamma\left(\tilde{t}_{h}\left(1+\tilde{k}_{h}\right)-\Gamma\right)}\right),
\end{aligned}
$$

and

$$
D=-\left(\frac{T_{h} \tilde{k}_{h} \tilde{E}_{h}\left(\Gamma-\tilde{E}_{h} \tilde{k}_{h}(1-(1 / \theta))\right.}{\tilde{E}_{h}\left(1+\tilde{k}_{h}\right)-\Gamma}\right) .
$$

It is also convenient to define a reduced coefficjent of performance (RCOP) given by $\eta\left(\tilde{t}_{h}, \tilde{t}_{1}\right)=\varepsilon\left(\tilde{t}_{h}, \tilde{t}_{1}\right) / \epsilon_{0}$, where $\boldsymbol{\epsilon}_{0}=\left(\left(T_{H} / T_{L}\right)-1\right)^{-1}$ is the quasistatic Carnot coefficient of performance.

Using $\eta\left(\tilde{t}_{1}, \tilde{E}_{h}\right), \tilde{E}_{h}=1 / \tilde{E}_{h}$, and $\tilde{f}_{1}=1 / \tilde{E}_{1}$, a three dimensional efficiency space is defined which consists of surfaces of constant $\theta$ and $\Gamma$ as shown in Figure 11 . Efficiency space surfaces composed of contours of constant cycling time vary continuously with the ratio $\tilde{t}_{h} / \tilde{t}_{1}$. These surfaces are obtained by plotting the ordered triple $\left(\tilde{f}_{1}, \tilde{f}_{h}, \eta\left(\tilde{f}_{1}, \tilde{f}_{h}\right)\right)$ as $\tilde{E}_{h} / \tilde{E}_{1}$ varies from 0 to $\infty$ within the feasible region defined below by constraints on $\eta\left(\tilde{t}_{h}, \tilde{t}_{1}\right)$. Paths within the efficiency space describing possible refrigeration cycle operating modes are referred to as 
$(10,0.0,0.0)$ :

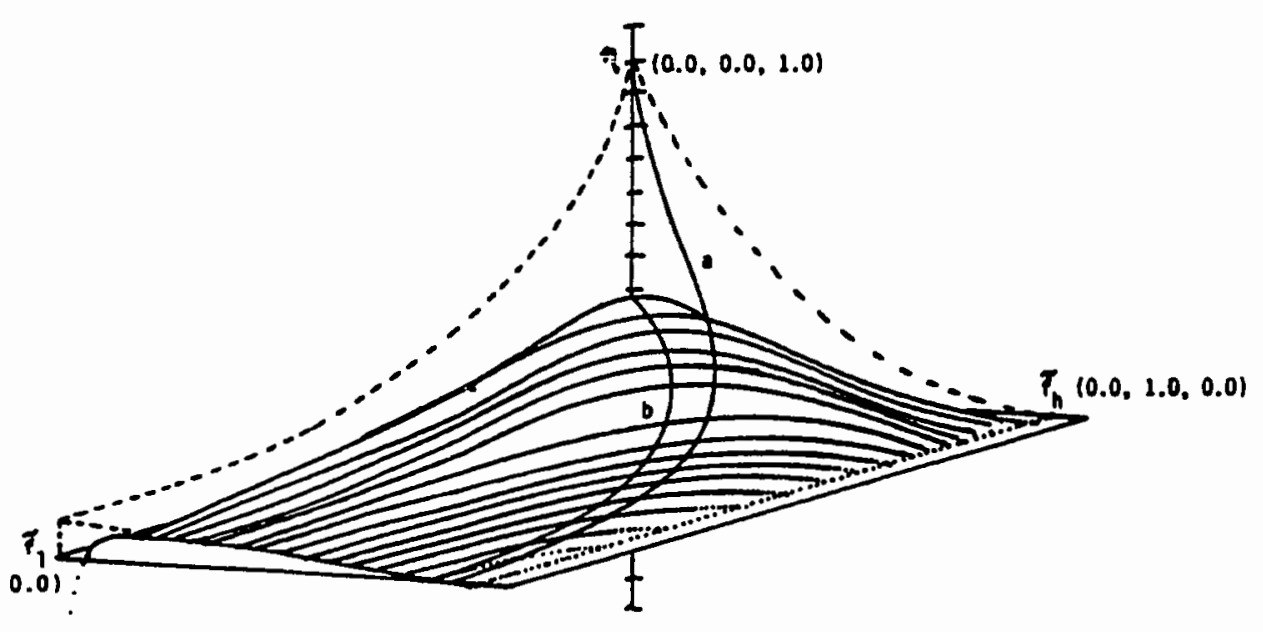

Figure 11. Three dimensional efficiency space for an FTCRC with Non-Ideal Heat Switches. The reduced COP (RCOP) $\eta\left(\tilde{f}_{h}, \tilde{F}_{1}\right)=\epsilon\left(\tilde{f}_{h}, \tilde{f}_{1}\right) / \varepsilon$ o is plotted vs the inverse times $\tilde{f}_{h}=1 / \tilde{E}_{h}$ and $\tilde{f}_{1}=1 / \tilde{t}_{1}$ spent along the low and high temperature heat transfer branches, respectively $(\Gamma=1$ and $\theta=3)$. The dashed curves show the outline of the same efficiency space surface for an FTCRC with ideal heat switches. Curve a represents the optimum efficiency trajectory for an FTCRC with ideal heat switches. Curve b represents the optimum efficiency trajectory representing the maximum efficiency operating mode. 
efficiency trajectories. An efficiency space as defined above contains efficiency trajectories for all possible operating modes of the FTCRC considered in this analysis.

At least three striking differences exist between efficiency space surfaces for FTCRC's with and without ideal heat switches. First, the efficiency space shown in Figure 11 representing the performance of an FTCRC with non-ideal heat switches is severely folded with respect to the efficiency surface shown in Figure 5 which represents an FTCRC with ideal heat switches. The degree to which the efficiency surface for the non-ideal case is folded indicates the severity of heat-leak through non-ideal switches. To make this latter comparison more visually apparent, dashed lines included in Figure 11 represent boundaries of an efficiency space surface for an FTCRC with perfect heat switches. In the limit of no heat-leak through the heat switches, the efficiency surfaces for FTCRC's with and without ideal heat switches coalesce.

Second, regimes of negative efficiency exist on the efficiency surface representing the FTCRC with non-ideal heat switches. These regimes are indicated in Figure 11 by the dotted lines. Negative efficiencies result from the inability of the refrigeration cycle to produce adequate cooling power to overcome heat leak through the thermal switches. In contrast, the cycling efficiency of an FTCRC with ideal heat switches is positive over the entire 
efficiency space as seen in Figure 5.

Third, the global maximum Carnot efficiency identified by the point $\left(\tilde{f}_{1}, \tilde{f}_{h}, \eta\left(\tilde{f}_{1}, \tilde{f}_{h}\right)\right)=(0.0,0.0,1.0)$ in both efficiency spaces is not attainable as long as there exists heat leaks through heat switches. With non-ideal heat switches there will always exist an operating frequency below which the heat leak due to imperfect thermal switching will dominate the ability of the refrigeration cycle to provide positive cooling power. Consequently, the FTCRC is restricted from attaining the global maximum quasistatic Carnot efficiency even in the quasistatic limit of zero cycling frequency.

The global maximum efficiency trajectory within the efficiency space for the FTCRC with non-ideal heat switches is obtained by maximizing $\epsilon\left(\tilde{E}_{h}, \tilde{E}_{1}\right)$ using an iterative numerical search method $(12,23)$. Global maxima along individual efficiency contours of constant cycling frequency are defined by the following simple rule: If $\hat{t}_{h}$ and $\hat{t}_{1}$ exist within the feasible region, and if for all $\tilde{t}_{h} \neq \hat{E}_{h}$ and $\tilde{E}_{1} \neq E_{1}, \eta\left(E_{h}, \tilde{t}_{1}\right)<\eta\left(\tilde{E}_{h}, \tilde{E}_{1}\right)$ along individual contours of constant cycling frequency, then $\hat{t}_{h}^{*}$ and $\hat{t}_{1}$ represent global optima which maximize $\epsilon\left(\tilde{t}_{h}, \tilde{t}_{1}\right)$.

Constraints defining the feasible region over which 
$\epsilon\left(\tilde{t}_{h}, \tilde{E}_{1}\right)$ is maximized are given by

$$
\begin{aligned}
& g_{1}=\tilde{t}_{1}>0, \\
& g_{2}=\tilde{t}_{h}\left(1+\tilde{k_{h}}\right)-\Gamma>0,
\end{aligned}
$$

and

$$
g_{3}=\tilde{\tau}-\left(\tilde{E}_{h}+\tilde{t}_{1}\right)=0
$$

Constraint $g_{1}$ insures that time spent during low temperature heat transfer is positive and nonzero. Constraint $g_{2}$ is necessary in order to maintain the correct sign of $Q_{H}$ the net heat exchanged with the high temperature thermal reservoir. The sign convention is such that $Q_{H}<0$ when heat leak from the high temperature reservoir is small and $Q_{H} \quad$ when heat leak out of the high temperature reservoir dominates heat transfer into the reservoir. Constraint $g_{3}$ requires that the cycle time devoted to heat transfer be proportional to the total cycle time as described above. Carrying out the numerical maximization of $\epsilon\left(\tilde{t}_{1}, \tilde{t}_{h}\right)$ over the feasible region, $\vec{t}_{h}$ and $\vec{E}_{1}$ are obtained. Using $\vec{t}_{h}$ and $\hat{t}_{1}$ in (4.29), curve b the optimum efficiency trajectory shown in Figure 11 is obtained, and the maximum efficiency operating mode is defined. For comparison, curve "a" in Figure 11 represents the optimum efficiency trajectory of an FTCRC with ideal heat switches. 
To examine the frequency dependent behavior of optimum efficiency space trajectories and further address the issues of negative efficiencies and the unattainability of $\varepsilon_{0}$, a family of optimum efficiency trajectories are plotted as functions of cycling frequency in Figure 12 for several values of the thermal reservoir temperature ratio $\theta=T_{H} / T_{L}$. Efficiency trajectory "a" represents an FTCRC with ideal switches. The efficiency of an FTCRC with ideal switches is always positive, reduces monotonically to zero with increasing cycling frequency, and approaches $\eta\left(\tilde{E}_{1}, \tilde{t}_{h}\right)=1.0$ the quasistatic carnot maximum in the $\tilde{f}=1 / \tilde{\tau} \rightarrow 01$ imit. Trajectories $b, c$, and $d$ which represent the non-ideal heat switch case attain global maxima at nonzero cycling frequencies and are negative in the limits of high and low frequency cycle operation. The occurrence of negative efficiencies may be explained as follows: recall that $\varepsilon\left(\tilde{t}_{1}, \tilde{t}_{h}\right)=Q_{L}\left(\tilde{t}_{1}, \tilde{t}_{h}\right) /-W_{n \theta t}\left(\tilde{t}_{1}, \tilde{t}_{h}\right)$, where $Q_{L}\left(\tilde{t}_{1}, \tilde{t}_{h}\right)$ the net heat extracted from the low temperature thermal reservoir may be positive, negative, or zero. The work required for one refrigeration cycle, $W_{\text {net }}\left(\tilde{t}_{1}, \tilde{t}_{h}\right)$, is always negative. Therefore, negative efficiencies occur due to $Q_{L}\left(\tilde{t}_{1}, \tilde{t}_{h}\right)=Q_{L 1}\left(\tilde{t}_{1}\right)+Q_{h L}\left(\tilde{t}_{h}\right)<0$, where $Q_{L 1}\left(\tilde{t}_{1}\right)$ and $Q_{h L}\left(\tilde{t}_{h}\right)$ represent competing mechanisms for extracting heat from and leaking heat back into the low temperature thermal 


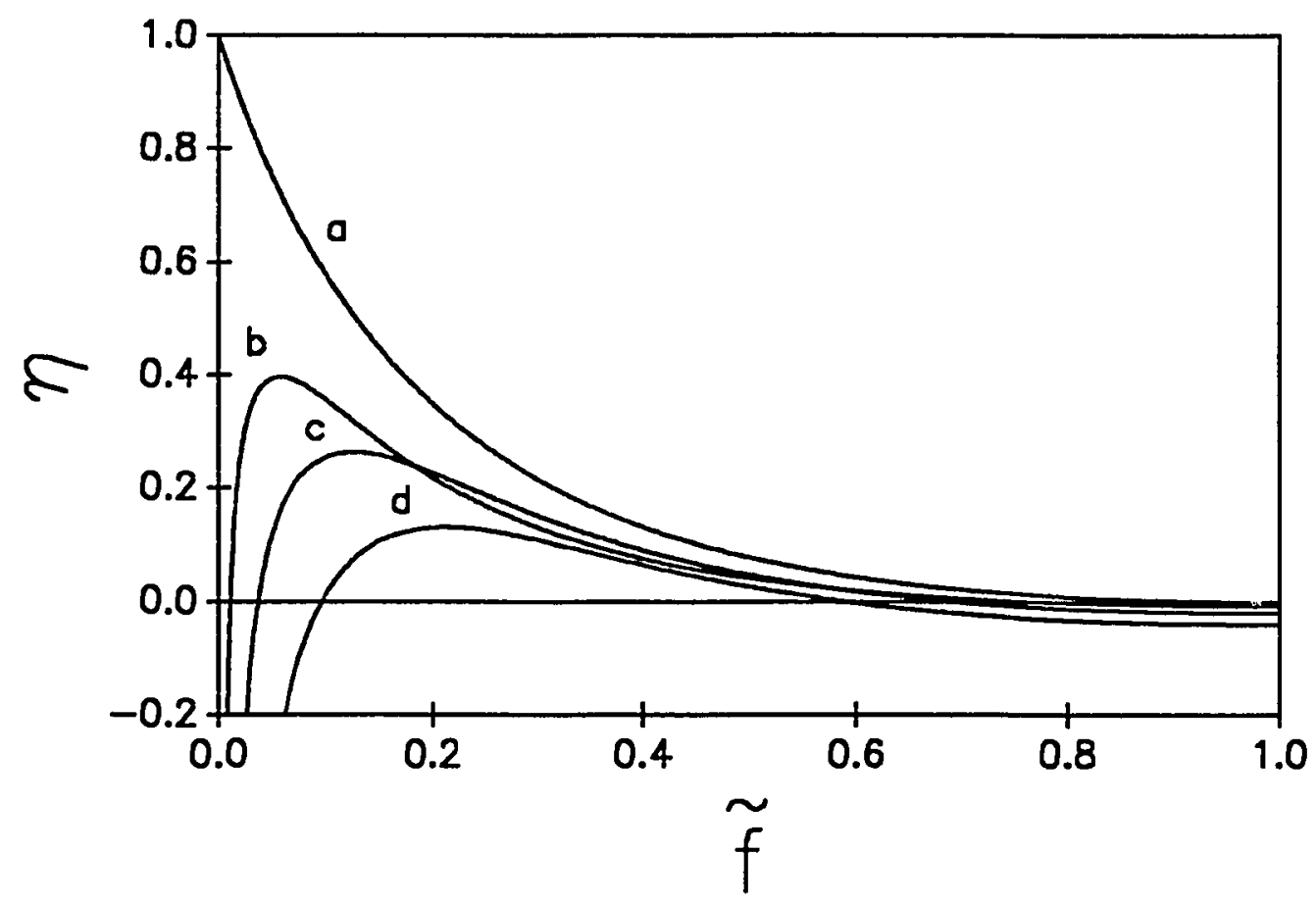

Figure 12. Reduced coefficients of performance, $\eta$, vs reduced cycling frequency, $\vec{f}$. Curve a represents the optimum efficiency trajectory for an FTCRC with ideal heat switches and $\theta=3.0 \quad(\Gamma=$ 1 ). Curves $b, c$, and $d$ represent $\eta$ the RCOP of an FTCRC with non-ideal heat switches and $\theta=1.5$, 3.0 , and 5.0 , respectively. 
reservoir, respectively. Because $Q_{h L}\left(E_{h}\right)$ is always negative, $Q_{L}\left(\tilde{t}_{1}, \tilde{t}_{h}\right)<0$ and consequently, $\epsilon\left(\tilde{t}_{1}, \tilde{t}_{h}\right)$ are negative when $\left|\ell_{L I}\left(\tilde{E}_{1}\right)\right|<\left|\ell_{h L}\left(\tilde{E}_{h}\right)\right|$.

Negative efficiencies at high and low operating frequencies are conveniently explained using $Q_{h L}=\dot{Q}_{h L} t_{h}$ for heat leak from the working fluid into the low temperature thermal reservoir and $Q_{L 1}=\dot{Q}_{L 1} t_{1}$ for heat extracted from the low temperature thermal reservoir into the working fluid. At high cycling frequencies, $Q_{h L}=\dot{Q}_{h L} t_{h} \neq 0$ and so heat leak remains finite, the positive heat flux out of the low temperature reservoir, $\dot{Q}_{L 1}$, remains nonzero, and $\tilde{E}_{1} \rightarrow 0$. Therefore, $Q_{L}\left(\tilde{t}_{1}, \tilde{t}_{h}\right)<0$ and consequently, $\eta\left(\tilde{t}_{1}, \tilde{t}_{h}\right)$ becomes negative due to the fact that time devoted to low temperature heat transfer is insufficient to allow enough cooling power to counteract heat leak. At low cycling frequencies $\tilde{t}_{1} \propto \tilde{t}_{h}, \dot{Q}_{h L}<0$, and $\dot{Q}_{L I} \propto 0$. Therefore, $Q_{L}\left(\tilde{t}_{1}, \tilde{t}_{h}\right)<0$, and negative efficiencies result once again because essentially no positive cooling power is produced. It should also be pointed out that this low frequency behavior results from the fact that heat flux associated with the leak is treated as a constant in the present FTCRC model. Consequently, there always is a low enough cycling 
frequency such that the ability of the refrigerator to produce positive cooling power becomes less than the flux of the leak.

In Figure 13, $\eta$ is shown as a function of $\tilde{P}_{c}$ the dimensionless cooling power. The cooling power is defined as $\tilde{P}_{c}=P_{c} / \sqrt{\alpha B} T_{L}=\left(Q_{L} / \tau\right) / \sqrt{\alpha B} T_{L}$, where as above $Q_{L}$ is the net heat extracted from the low temperature thermal reservoir and $\tau$ is the cycling period of the refrigeration cycle. Curve a represents an FTCRC with no heat-leak for $\theta=3.0$ and attains a single maximum with respect to cooling power. Curves b, $c$, and $d$ represent refrigeration cycles with heatleaks for $\theta=1.5,3.0$, and 5.0, respectively. Note that curves representing the refrigeration cycle with heat-leak attain maxima with respect to both efficiency and cooling power.

In Table II, $\varepsilon_{m}^{\prime}$ the efficiency evaluated at maximum cooling power and $\epsilon^{* /}$ the global maximum efficiency obtained above for an FTCRC with non-ideal heat switches are compared to $\epsilon_{0}$ the quasistatic Carnot efficiency and $\epsilon_{m}$ the efficiency at maximum cooling power of an FTCRC with ideal heat switches. $\epsilon_{m}^{\prime}$ and $\epsilon^{* /}$ are not being directly compared with the experimental data because the purpose of this research is not to predict the efficiency of specific refrigeration cycles. Rather, interest lies in establishing 


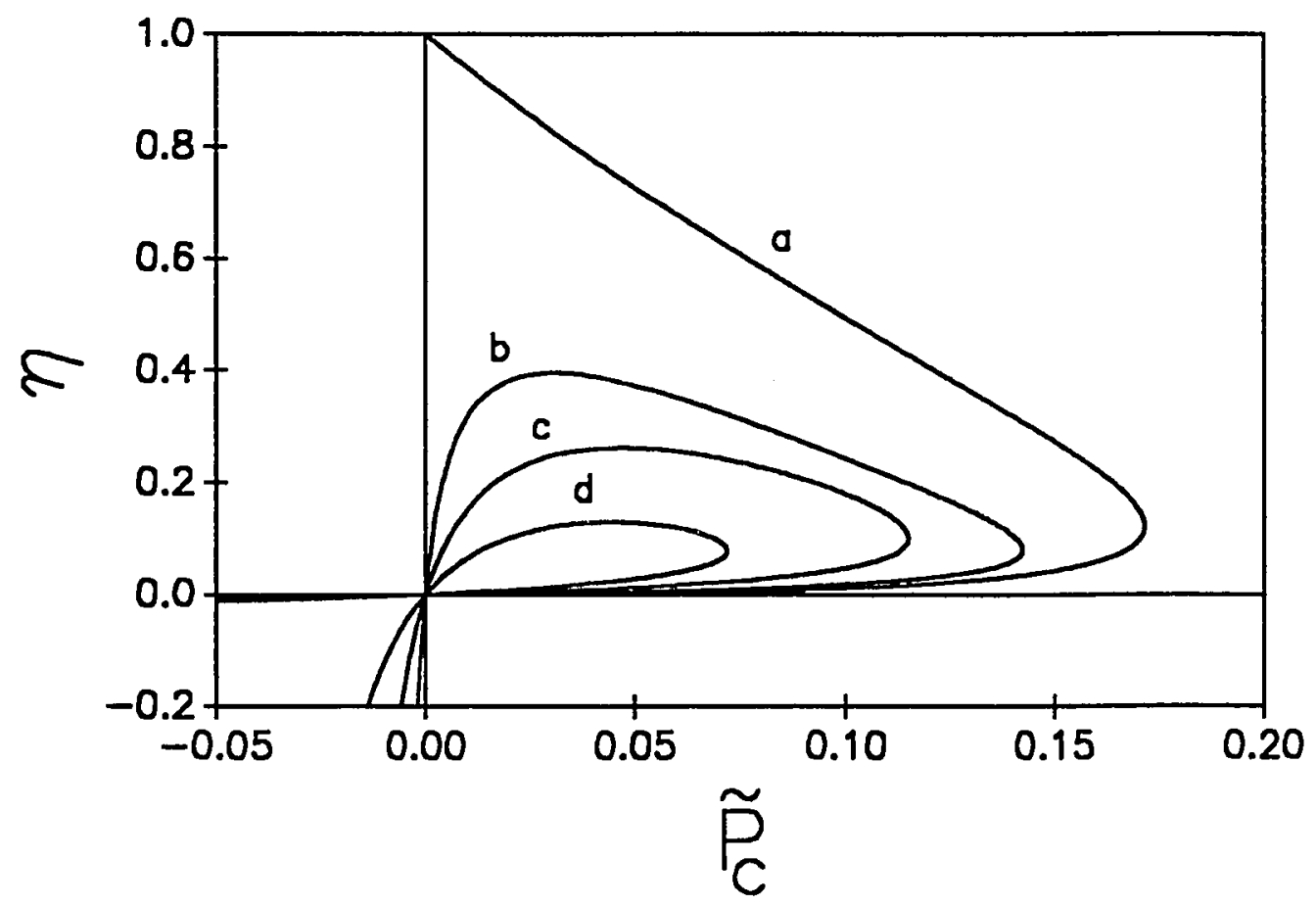

Figure 13. Reduced coefficient of performance, $\eta$, vs reduced cooling power $\tilde{P}_{c}$. Curve a

represents an FTCRC with ideal heat switches $(\Gamma=$ 1). Curves $b, c$, and d represent an FTCRC with non-ideal heat switches and $\theta=1.5,3.0$, and 5.0, respectively. 
TABLE II

COMPARISON OF THE QUASISTATIC CARNOT COP

WITH THE COP AT FINITE COOLING POWER

OF AN FTCRC WITH NON-IDEAL

HEAT SWITCHES

\begin{tabular}{|c|c|c|c|c|c|c||}
\hline $\begin{array}{c}\text { Refrigeration } \\
\text { Cycle }\end{array}$ & $\frac{\mathrm{T}_{\mathrm{H}}}{\mathrm{T}_{\mathrm{L}}}$ & $\begin{array}{c}\epsilon \\
\text { Carnot }\end{array}$ & $\begin{array}{c}\epsilon_{\mathrm{m}} \\
\text { Ideal } \\
\text { Heat } \\
\text { Switches }\end{array}$ & $\begin{array}{c}\epsilon_{\mathrm{m}}^{\prime} \\
\text { Non-Ideal } \\
\text { Heat } \\
\text { Switches }\end{array}$ & $\begin{array}{c}\epsilon^{* \prime} \\
\text { Non-Ideal } \\
\text { Heat } \\
\text { Switches }\end{array}$ & $\begin{array}{c}\epsilon \\
\text { Observed }\end{array}$ \\
\hline $\begin{array}{c}\text { Reciprocating } \\
\text { Magnetic (21) }\end{array}$ & 2.00 & 1.0000 & 0.0938 & 0.0898 & 0.3544 & 0.0630 \\
\hline$(* \mathrm{~B}=5 \mathrm{~T})$ & 2.21 & 0.8264 & 0.0842 & 0.0792 & 0.2803 & 0.0777 \\
\hline (B $=2 \mathrm{~T})$ & 2.21 & 0.8264 & 0.0842 & 0.0792 & 0.2803 & 0.0355 \\
\hline (B $=5 T)$ & 2.33 & 0.7500 & 0.0794 & 0.0791 & 0.2442 & 0.0862 \\
\hline Static Magnetic(21)
\end{tabular}

* "B" represents the externally applied magnetic field. 
bounds on thermal efficiency that are better than the quasistatic carnot bounds.

For a given experiment, say the reciprocating magnetic refrigeration cycle with $T_{H} / T_{L}=2.21$ and $B=2.0$ Tesla, an experimental efficiency of $\epsilon=0.0777$ was obtained. The quasistatic carnot bound is $\epsilon_{0}=0.8264$. The new bound obtained in Chapter II based on the performance of an FTCRC with ideal heat switches is $\epsilon_{m}=0.0842$. This latter CoP reduced the bound on thermal efficiency by more than a factor of 9 and still bounded the experimental efficiency. Extending this latter comparison to include $\epsilon_{m}^{\prime}$, the efficiency at maximum cooling power within the maximum efficiency operating mode is $\epsilon_{m}^{\prime}=0.0792$. Relative to the Carnot bound, $\epsilon_{m}^{\prime}$ reduces the bound on thermal efficiency of refrigeration cycles by a factor of 10 while still bounding the experimental magnetic refrigeration cycle efficiency. In fact, $\epsilon_{m}^{\prime}$ functions as an improved bound on thermal efficiency in three of the cases presented in Table II and functions as a bound in the fourth case within experimental error. Within this same example, the global maximum efficiency obtained above for an FTCRC with non-ideal heat switches is $\epsilon^{* \prime}=0.2803$. The global bounding efficiency, $e^{* \prime}$, reduces the bound on efficiency by about a factor of 3 
and bounds all of the experimental efficiencies presented in Table II. It is encouraging therefore, based on these limited comparisons, that the results obtained in this chapter may represent further improvement of bounds on the global maximum thermal efficiency and the efficiency at maximum cooling power of certain refrigeration cycles which produce nonzero cooling power.

\section{SUMMARY AND CONCLUSION}

A model endoreversible finite time carnot refrigeration cycle with non-ideal heat switches has been numerically optimized. In contrast to the traditional quasistatic carnot refrigeration cycle, the FTCRC with non-ideal thermal switches operates irreversibly over a continuous range of cycling times, produces finite cooling power, and experiences heat leaks through the heat switches. The principal results are 1) the numerically obtained global maximum coefficient of performance and 2) the coefficient of performance evaluated at maximum cooling power within the maximum efficiency operating mode. These new bounds on refrigeration cycle thermal efficiency may provide improved estimates of the bound on the thermal efficiency of irreversible refrigeration cycles with non-ideal thermal switches that maximize efficiency while producing cooling power. It is hoped that the finite time thermodynamic analyses that has been carried out here will prove to be 
helpful in furthering our understanding of the thermodynamics of irreversible refrigeration cycles. 


\section{REFERENCES}

1. Energy and Power; a) Energy and Power, c. Starr, pp. 319, b) The Conversion of Energy, C.M. Summers, pp. 95-109, Scientific America Inc. (1971)

2. F.L. Curzon and B. Ahlborn, Am.J.Phys. 43, 22 (1975)

3. P. Salamon and A. Nitzan, J. Chem. Phys. 74, 3546 (1981)

4. P. Salamon, A. Nitzan, B. Andresen, and R.S. Berry, Phys. Rev. A 21, 2115 (1980)

5. M. Rubin, Phys. Rev. A 19, 1272, 1277 (1979)

6. B. Andresen, R.S. Berry, M. Ondrechen, and P. Salamon, Acc. Chem. Res. 17, 266-271 (1984)

7. B. Andresen, P. Salamon, and R.S. Berry, Phys. Today, September 1984

8. B. Andresen, P. Salamon, and R.S. Berry, J. Chem. Phys. 66,1571 (1977)

9. D. Gutkowicz-Krusin, I. Procaccia, and J. Ross, J. Chem. Phys. 69, 3898 (1978)

10. M. Mozurkewick and R.S, Berry, J. Appl. Phys. 53, 34 (1982)

11. Y.B. Band, O. Kafri, and P. Salamon, J. Appl. Phys. 53, 8,29 (1982)

12. L.R. Foulds, optimization Techniques, Springer-Verlag (1981)

13. K. Lancaster, Mathematical Economics, Dover, (1968)

14. Y. Hakuraku and H. Ogata, Cryogenics, 26, March (1986)

15. W.A. Steyert, J. Appl. Phys. 49 (3), 1216 (1978)

16. J.D. Walters and J.S. Semura, "Optimal performance of a finite time carnot refrigeration cycle," Bull. Am. Phys. Soc. 34, 119 (1989); J.D. Walters and J.S. Semura, to be published 
17. B. Andresen, R.S. Berry, A. Nitzan, and P. Salamon, Phys. Rev. A 15, 2086-2093 (1977)

18. M.H. Rubin and B. Andresen, J. Appl. Phys. 53, 1-7 (1982)

19. See, for example, H.B. Callen, Thermodynamics and an Introduction to Thermostatics, second Edition, (Wiley, New York, NY, 1985) p. 115

20. R. Radenbaugh, "Refrigeration Fundamentals: A view toward new refrigeration systems," NBS SP \#508, 37 (1978)

21. C. Delpuech, R. Beranger, G. Bon Mardion, G. Claudet, and A.A. Lacaze, Cryogenics 10, 579-584 (1981); To include the efficiencies reported in this reference, we have made the following conversion: $\epsilon$ $=(\theta-1) /\left(\left(\theta / \eta^{\prime}\right)-1\right)$, where $\theta=\mathrm{T}_{\mathrm{H}} / \mathrm{T}_{\mathrm{L}}$ and $\eta^{\prime}$ is the reported efficiency.

22. Z.Yan and Wu Li, Physics (in Chinese) 13, 768 (1984); J. Appl. Phys. 63, 4795-4798 (1988); Phys. Rev. A 39 , 4140-4147 (1989)

23. D.A. Pierre, optimization Theory with Applications, (Dover, New York, 1969) 
APPENDIX A

CONVEXITY/CONCAVITY OF OBJECTIVE FUNCTIONS 
In this appendix, it is shown that the objective functions representing the cooling power, efficiency, power consumption, and irreversible entropy production are suitably concave or convex with respect to $\tilde{E}_{h}$ and $E_{1}$ over the feasible region defined by Eqs. (3.30a) - (3.30c) in order to fulfill the Kuhn-Tucker (KT) optimization conditions.

\section{COOLING POWER}

Using Eq. (3.13), the cooling power of the FTCRC is given by

$$
P_{c}=\frac{Q_{1}}{\tau}=\frac{\sqrt{\alpha B} T_{L} \Gamma \tilde{E}_{1}}{\tilde{\tau}\left(\Gamma \tilde{E}_{1}+1\right)} .
$$

Because $P_{c}$ depends only on $\tilde{E}_{1}$, it is sufficient to show that $P_{c}$ is concave with respect to $\tilde{t}_{1}$ in order that it satisfy the extended KT conditions. Evaluating the second derivative of $P_{c}$ with respect to $\tilde{E}_{1}$ and holding the dimensionless cycle period $\tilde{\tau}$ constant, the following is obtained:

$$
\frac{\partial^{2} P_{c}}{\partial \tilde{t}_{1}^{2}}=\frac{-2 \sqrt{\alpha 1 S \Gamma^{2}} T_{L}}{\tilde{\tau}\left(\Gamma \tilde{t}_{1}+1\right)^{3}}<0 .
$$

Because $\partial^{2} P_{c} / \partial \tilde{t}_{1}^{2}<0$, the cooling power function is strictly 
concave and a global Kuhn-Tucker maximum exists within the feasible region.

\section{EFFICIENCY}

The efficiency of the FTCRC is measured by the following coefficient of performance (COP):

$$
\epsilon\left(\tilde{t}_{h}, \tilde{t}_{1}\right)=\frac{Q_{1}}{-W_{n e t}}=\left(\frac{\theta \tilde{t}_{h}\left(\Gamma \tilde{t}_{1}+1\right)}{\Gamma \tilde{t}_{1}\left(\tilde{t}_{h}-\Gamma\right)}-1\right)^{-1} \cdot
$$

It has been shown in Chapter III that maximizing $x\left(\tilde{t}_{1}, \tilde{t}_{h}\right)$ $=-Q_{1} / Q_{h}$ is equivalent to maximizing $\epsilon$. In maximizing $X\left(\tilde{E}_{1}, \tilde{t}_{h}\right)$, the optimum ratio of $\tilde{t}_{h} / \tilde{t}_{1}$ is actually being obtained along contours of constant cycling frequency within the efficiency space shown in Figure 2. Therefore, to determine if $X\left(\tilde{t}_{1}, \tilde{t}_{h}\right)$ is concave, one examines the way in which $X\left(\tilde{E}_{1}, \tilde{E}_{h}\right)$ varies with the ratio $\tilde{E}_{h} / \tilde{t}_{1}$ along contours of constant frequency. Making the substitution $r=\tilde{t}_{h} / \tilde{t}_{1}$

$$
X(r)=\frac{r(\tilde{\tau}-\Gamma)-1}{\theta\left(r^{2}+r(1+\Gamma \tilde{\tau})\right)}
$$

The second derivative of $\mathrm{X}$ with respect to $\mathrm{r}$ is given by

$$
\frac{d^{2} X}{d r^{2}}=\frac{2 \Gamma r^{2}(\tilde{\tau}-\Gamma)}{\theta(r+\Gamma \tilde{\tau}+1)}
$$




$$
-\frac{2 \Gamma^{2}\left(3 r^{2}+3(1+\Gamma \tilde{\tau}) \Gamma+(1+\Gamma \tilde{\tau})^{2}\right)}{\theta\left(I^{2}+\Gamma(1+\Gamma \tilde{\tau})\right)}
$$

In order for $X(r)$ to be strictly concave with respect to $r$, it is required that $d^{2} X / d r^{2}<0$. The sign of $d^{2} X / d r^{2}$ can be determined by examining the cubic inside the brackets of Eq. (A5). In the present case, because of the negative sign in (A5), $X(I)$ is not in general a strictly concave function for arbitrary values of $\tilde{\tau}$ and $\Gamma$. This means that determining the concavity of $X(r)$ must be done by exiinining the sign of $d^{2} X / d r^{2}$ on a case by case basis for specific values of $\Gamma$ and $\tilde{\tau}$. In the special symmetric heat transfer case of $\Gamma=1$, for example, as is shown in Figure 2, Eq. (A5) reduces to

$$
\frac{d^{2} X}{d r^{2}}=\frac{-2\left(3 r^{2}+3(1+\tilde{\tau}) r+(I+\tilde{\tau})^{2}\right)}{\theta\left(r^{2}+r(1+\tilde{\tau})\right)}<0 .
$$

Therefore, for $\Gamma=1, X(r)$ is a strictly concave function along contours of constant cycling frequency, and we are assured that the Kuhn-Tucker optimization produces a global maximum. In addition to the $\Gamma=1$ special case, because $\epsilon$ is a well behaved function and because the global maximum quasistatic carnot efficiency must always be attained along the concave $\tilde{\tau}=\infty$ contour, it is not unreasonable to expect that some nonzero number of contours of constant frequency will always be suitably concave to satisfy the Kuhn-Tucker conditions for certain values of $\tilde{\tau}$ for $\Gamma \neq 1$. This last 
statement has been verified numerically for several values of $\Gamma$ such that $0<\Gamma \neq 1$.

POWER CONSUMPTION

The power consumption of the FTCRC is given by

$$
P=\frac{-\left(Q_{h}+Q_{1}\right)}{\tau}=\frac{\sqrt{\alpha \beta}}{\tilde{\tau}}\left(\frac{T_{H} \tilde{t}_{h}}{\left(\tilde{t}_{h}-\Gamma\right)}-\frac{T_{L} \Gamma \tilde{t}_{1}}{\left(\Gamma \tilde{t}_{1}+1\right)}\right) \text {. }
$$

To determine the concavity of $P$, the determinant of the matrix of second derivatives of $P$ with respect to $\tilde{t}_{1}$ and $\tilde{t}_{h}$ is examined. This latter determinant is the Hessian of the power function which is given by

$$
H=\frac{\partial^{2} P}{\partial \hat{t}_{h}^{2}} \frac{\partial^{2} P}{\partial \tilde{t}_{1}^{2}}-\left(\frac{\partial^{2} P}{\partial \tilde{t}_{1} \partial \tilde{t}_{h}}\right)^{2} \text {. }
$$

If the Hessian is positive definite (i.e., if $H>0$ ), the power consumption function is strictly convex (14).

The following second derivatives make up the matrix elements used to calculate the Hessian of $P$ :

$$
\begin{aligned}
& \frac{\partial^{2} P}{\partial \tilde{t}_{1}^{2}}=\frac{2 \sqrt{\alpha \mathcal{B} \Gamma^{2} T_{L}}}{\tilde{\tau}\left(\Gamma \tilde{t}_{1}+1\right)^{3}}<0, \\
& \frac{\partial^{2} P}{\partial \tilde{t}_{h}^{2}}=\frac{2 \sqrt{\alpha \mathcal{B} \Gamma T_{H}}}{\tilde{\tau}\left(\tilde{t}_{h}-\Gamma\right)^{3}}>0,
\end{aligned}
$$


and

$$
\frac{\partial^{2} P}{\partial \tilde{t}_{h} \partial \tilde{t}_{1}}=\frac{\partial^{2} P}{\partial \tilde{t}_{1} \partial \tilde{t}_{h}}=0
$$

Using (A9) - (A11) in (A8),

$$
H=\frac{4 \sqrt{\alpha \mathcal{B} \Gamma^{3} T_{H} T_{L}}}{\tilde{\tau}^{2}\left(\Gamma \tilde{t}_{I}+1\right)^{3}\left(\tilde{t}_{h}+1\right)^{3}}>0 .
$$

Because the Hessian of (A12) is positive definite, the power consumption function is strictly convex.

\section{IRREVERSIBLE ENTROPY PRODUCTION}

The irreversible entropy produced per cycle of the FTCRC is given by

$$
\begin{aligned}
\Delta S_{I}= & -\left(\frac{Q_{h}}{T_{H}}+\frac{Q_{I}}{T_{L}}\right) \\
& =-\Delta S\left(\frac{-\tilde{t}_{h}}{T_{H}\left(\tilde{t}_{h}-\Gamma\right)}+\frac{\Gamma \tilde{t}_{1}}{T_{L}\left(\Gamma \tilde{t}_{1}+1\right)}\right) .
\end{aligned}
$$

In order to use the Hessian method to determine if the irreversible entropy function is strictly convex, the following derivatives are obtained:

$$
\frac{\partial^{2} \Delta S_{I}}{\partial \hat{t}_{1}^{2}}=\frac{2 \Delta S \Gamma}{\left(\Gamma \tilde{t}_{1}+1\right)^{3}}>0,
$$




$$
\begin{gathered}
\frac{\partial^{2} \Delta S_{I}}{\partial \tilde{t}_{h}^{2}}=\frac{2 \Delta S \Gamma}{\left(\tilde{t}_{h}-\Gamma\right)^{3}}>0, \\
\frac{\partial^{2} \Delta S_{I}}{\partial \tilde{t}_{h} \partial \tilde{t}_{I}}=\frac{\partial^{2} \Delta S_{I}}{\partial \tilde{t}_{1} \partial \tilde{t}_{h}}=0 .
\end{gathered}
$$

The Hessian is given by

$$
\begin{aligned}
H= & \frac{\partial^{2} \Delta S_{I}}{\partial \tilde{t}_{h}^{2}} \frac{\partial^{2} \Delta S_{I}}{\partial \tilde{t}_{1}^{2}}-\left(\frac{\partial^{2} \Delta S_{I}}{\partial \tilde{t}_{1} \partial \tilde{t}_{h}}\right)^{2} \\
& =\frac{4 \Delta S^{2} \Gamma^{2}}{\left(\Gamma \tilde{t}_{1}+1\right)^{3}\left(\tilde{t}_{h}-\Gamma\right)^{3}}>0 .
\end{aligned}
$$

Because $H>0$, the irreversible entropy production objective function is strictly convex.

In summary, the efficiency function is strictly concave for $\Gamma=1$ and a simple expression that can be used to test the concavity of the efficiency for $\Gamma \neq 1$ has been obtained. In addition, the cooling power function is strictly concave and the power consumption and irreversible entropy production are strictly convex functions in fulfillment of the Kuhn-Tucker global optimization conditions. 


\author{
APPENDIX B \\ EQUIVALENCE OF OPTIMIZING THE NET HEAT TRANSFER \\ INTO THE WORKING FLUID AND THE NET \\ HEAT TRANSFER INTO THE EXTERNAL \\ HEAT RESERVOIRS
}


The efficiency of an FTCRC with heat leak due to nonideal heat switches was defined in Eq. (4.8) as

$$
e\left(t_{1}, t_{h}\right)=\frac{Q_{L}\left(t_{1}, t_{h}\right)}{-W_{n \in t}\left(t_{1}, t_{h}\right)}=\left(\left(\frac{-Q_{H}\left(t_{1}, t_{h}\right)}{Q_{L}\left(t_{1}, t_{h}\right)}\right)-1\right)^{-1}
$$

where $Q_{H}\left(t_{h}, t_{1}\right)$ and $Q_{L}\left(t_{h}, t_{1}\right)$ are the net heats exhausted to and absorbed from the high and low temperature thermal reservoirs, respectively. In addition, it was shown that $\epsilon\left(t_{1}, t_{h}\right)$ is maximized when $\left|Q_{H}\left(t_{h}, t_{1}\right)\right|$ and $\left|Q_{L}\left(t_{h}, t_{1}\right)\right|$ are minimized and maximized, respectively. In maximizing $\epsilon\left(t_{1}, t_{h}\right)$ within Chapter IV however, $\left|Q_{h}\left(t_{h}, t_{1}\right)\right|$ and $\left|Q_{1}\left(t_{h}, t_{1}\right)\right|$ the net heats rejected from and absorbed into the working fluid have been extremized rather than $Q_{H}\left(t_{h}, t_{1}\right)$ and $Q_{L}\left(t_{h}, t_{1}\right)$. This alternative optimization was carried out for mathematical convenience and to maintain consistency with previous optimizations. The purpose of this appendix is to shown that optimizing $Q_{h}\left(t_{h}, t_{1}\right)$ and $Q_{1}\left(t_{h}, t_{1}\right)$ does in fact yield the same optimum heat transfer solution as does optimizing $Q_{H}\left(t_{h}, t_{1}\right)$ and $Q_{L}\left(\tilde{t}_{h}, \tilde{t}_{1}\right)$.

The general form of the Lagrangian used to optimize heat transfer into and out of thermal reservoirs is given by $\mathscr{L}=Q_{R}-\lambda_{R}^{(1)} \Delta S_{R}-\lambda_{R}^{(2)} \Delta S_{R^{\prime}}$

where $Q_{R}=Q_{L}\left(t_{1}, t_{h}\right)=Q_{h L}\left(t_{h}\right)+Q_{L I}\left(t_{1}\right)$ or $Q_{R}=Q_{H}\left(t_{1}, t_{h}\right)=Q_{H h}\left(t_{h}\right)+Q_{H 1}\left(t_{1}\right)$. In addition, $\Delta S_{R^{\prime}}=\Delta S_{H 1}$ or 
$\Delta S_{R^{\prime}}=\Delta S_{h L}$ and $\Delta S_{R}=\Delta S_{L I}$ or $\Delta S_{R}=\Delta S_{h H}$ are the entropies

generated in the working fluid due to heat leak and normal

heat transfer, respectively, and the $\lambda$ 's are Lagrange multipliers.

Entropy generated in the working fluid during low temperature heat transfer is given by

$$
\begin{aligned}
\Delta S_{L I} & =\int_{0}^{t_{1}} \frac{Q_{L I}\left(t_{1}\right)}{T_{1}\left(t_{1}\right)} d t \\
& =\int_{0}^{t_{1}} \frac{\alpha\left(T_{L}-T_{1}(t)\right)}{T_{1}(t)} d t=\xi_{1} \Delta S,
\end{aligned}
$$

and

$$
\begin{aligned}
\Delta S_{H I} & =\int_{0}^{t_{1}} \frac{Q_{H I}\left(t_{1}\right)}{T_{1}\left(t_{1}\right)} d t \\
& =\int_{0}^{t_{1}} \frac{k_{1}\left(T_{H}-T_{1}(t)\right)}{T_{1}(t)} d t=\left(1-\xi_{1}\right) \Delta S,
\end{aligned}
$$

where

$$
\Delta S_{L 1}+\Delta S_{H 1}=\Delta S=\text { const. }
$$

Entropy generated in the working fluid during high temperature heat transfer is given by

$$
\begin{aligned}
\Delta S_{h H} & =\int_{0}^{t_{h}} \frac{Q_{h H}\left(t_{h}\right)}{T_{h}\left(t_{h}\right)} d t \\
& =\int_{0}^{t_{h}} \frac{B\left(T_{H}-T_{h}(t)\right)}{T_{h}(t)} d t=-\xi_{h} \Delta S,
\end{aligned}
$$


and

$$
\begin{aligned}
\Delta S_{h L} & =\int_{0}^{t_{h}} \frac{Q_{h L}\left(t_{h}\right)}{T_{h}\left(t_{h}\right)} d t \\
& =\int_{0}^{t_{h}} \frac{k_{h}\left(T_{L}-T_{h}\left(t_{h}\right)\right)}{T_{h}\left(t_{h}\right)} d t=-\left(1-\xi_{h}\right) \Delta S,
\end{aligned}
$$

where

$$
\Delta S_{h H}+\Delta S_{h L}=-\Delta S=\text { Const. }
$$

The parameters $0<\xi_{1} \leq 1.0$ and $0<\xi_{h} \leq 1.0$ define the fractional change in entropy of the working fluid which is not due to the heat leak, and terms containing $(1-\xi)$ define changes in entropy of the working fluid which is due to heat leak. In the limit of $\xi=1.0$ no heat leak occurs and heat switches function ideally. In addition, $\Delta S_{1}+\Delta S_{h}=0$ in fulfillment of the endoreversibility condition.

The Lagrangian used to optimize $Q_{L}\left(t_{1}, t_{h}\right)$ is given by

$$
\mathscr{L}^{(1)}=Q_{L}\left(t_{1}, t_{h}\right)-\lambda_{1}^{(1)} \Delta S_{L I}-\lambda_{1}^{(2)} \Delta S_{h L}
$$

and alternatively, using $Q_{L}\left(t_{1}, t_{h}\right)=Q_{L 1}\left(t_{1}\right)+Q_{h L}\left(t_{h}\right)$, is given by

$$
\mathscr{L}^{(1)}=\left(Q_{h L}\left(t_{h}\right)+Q_{L I}\left(t_{1}\right)\right)-\lambda_{1}^{(1)} \Delta S_{L I}-\lambda_{1}^{(2)} \Delta S_{h L}
$$

Using (B9) and solving $\partial \mathscr{Q}^{(1)} / \partial T_{1}=0$ and $\partial \mathscr{L}^{(1)} / \partial T_{h}=0$, optimum temperatures of the working fluid during heat transfer are 
given by

$$
T_{1}^{(1)}(t)=\sqrt{\lambda_{1}^{(1)} \xi_{1} T_{L}}=\text { const. }=T_{1}^{(1)}
$$

and

$$
T_{h}^{(1)}(t)=\sqrt{\lambda_{1}^{(2)}\left(1-\xi_{h}\right) T_{L}}=\text { const. }=T_{h}^{(1)} .
$$

The Lagrange multipliers are

$$
\lambda_{1}^{(1)}=\frac{\xi_{1}\left(T_{1}^{(1)}\right)^{2}}{T_{L}}>0,
$$

and

$$
\lambda_{1}^{(2)}=\frac{\left(1-\xi_{h}\right)\left(T_{h}^{(1)}\right)^{2}}{T_{L}}>0 .
$$

For the Legendre condition, the following are obtained:

$$
\frac{\partial^{2} \mathscr{L}^{(1)}}{\partial\left(T_{1}^{(1)}\right)^{2}}=-\frac{2 \alpha \lambda_{1}^{(1)} \xi_{1} T_{L}}{\left(T_{1}^{(1)}\right)^{3}}<0,
$$

and

$$
\frac{\partial^{2} \mathscr{L}^{(1)}}{\partial\left(T_{h}^{(1)}\right)^{2}}=-\frac{2 k_{h} \lambda_{1}^{(2)}\left(1-\xi_{h}\right) T_{L}}{\left(T_{h}^{(1)}\right)^{3}}<0 .
$$

The free endpoint conditions

$$
\partial \mathscr{L}^{(1)} /\left.\partial \dot{T}_{1}^{(1)}\right|_{t=0}=\partial \mathscr{L}^{(1)} /\left.\partial \dot{T}_{1}^{(1)}\right|_{t=t_{1}}=0,
$$

and

$$
\partial \mathscr{L}^{(1)} /\left.\partial \dot{T}_{h}^{(1)}\right|_{t=0}=\partial \mathscr{L}^{(1)} / \partial \dot{T}_{h}^{(1)} \quad t=t_{h}=0
$$


are automatically satisfied. Therefore, using Eqs. (B3) and (B4), optimum temperatures of the working during heat transfer are given by

$$
T_{1}^{(1)}=\frac{T_{L}}{\left(1+\frac{\xi_{1} \Delta S}{\alpha t_{1}}\right)}
$$

and

$$
T_{h}^{(1)}=\frac{T_{L}}{\left(1-\frac{\left(1-\xi_{h}\right) \Delta S}{k_{h} t_{h}}\right)},
$$

where $\xi_{1}$ and $\xi_{h}$ are as yet undetermined.

The Lagrangian used to optimize $Q_{H}\left(t_{1}, t_{h}\right)$ is given by

$$
\mathscr{L}^{(2)}=Q_{H}\left(t_{1}, t_{h}\right)-\lambda_{h}^{(1)} \Delta S_{h H}-\lambda_{h}^{(2)} \Delta S_{H 1},
$$

and alternatively, using $Q_{H}\left(t_{1}, t_{h}\right)=Q_{H h}\left(t_{h}\right)+Q_{H 1}\left(t_{1}\right)$, is given by

$$
\mathscr{L}^{(2)}=\left(Q_{h H}\left(t_{h}\right)+Q_{H I}\left(t_{1}\right)\right)-\lambda_{h}^{(1)} \Delta S_{h H}-\lambda_{h}^{(2)} \Delta S_{H 1} .
$$

Using (B21) and solving $\partial \mathscr{L}^{(2)} / \partial T_{1}=0$ and $\partial \mathscr{L}^{(2)} / \partial T_{h}=0$, optimum temperatures of the working fluid during heat transfer are given by

$$
T_{1}^{(2)}(t)=\sqrt{\lambda_{h}^{(1)}\left(1-\xi_{1}\right) T_{H}}=\text { const. }=T_{1}^{(2)} \text {, }
$$

and 


$$
T_{h}^{(2)}(t)=\sqrt{\lambda_{h}^{(2)} \xi_{h} T_{H}}=\text { const. }=T_{h}^{(2)} .
$$

The Lagrange multipliers are

$$
\lambda_{h}^{(1)}=\frac{\left(1-\xi_{1}\right)\left(T_{1}^{(2)}\right)^{2}}{T_{H}}>0,
$$

and

$$
\lambda_{h}^{(2)}=\frac{\xi_{h}\left(T_{h}^{(2)}\right)^{2}}{T_{H}}>0 .
$$

For the Legendre condition, the following are obtained:

$$
\frac{\partial^{2} \mathscr{L}^{(2)}}{\partial\left(T_{I}^{(2)}\right)^{2}}=-\frac{2 k_{1} \lambda_{h}^{(1)}\left(1-\xi_{1}\right) T_{H}}{\left(T_{I}^{(2)}\right)^{3}}<0,
$$

and

$$
\frac{\partial^{2} \mathscr{L}^{(2)}}{\partial\left(T_{h}^{(2)}\right)^{2}}=-\frac{2 B \lambda_{h}^{(2)} \xi_{h} T_{H}}{\left(T_{h}^{(2)}\right)^{3}}<0 .
$$

Again, the free endpoint conditions

$$
\partial \mathscr{L}^{(2)} /\left.\partial \dot{T}_{1}^{(2)}\right|_{t=0}=\partial \mathscr{L}^{(2)} /\left.\partial \dot{T}_{1}^{(2)}\right|_{t=t_{1}}=0,
$$

and

$$
\partial \mathscr{Q}^{(2)} /\left.\partial \dot{T}_{h}^{(2)}\right|_{t=0}=\partial \mathscr{Q}^{(2)} /\left.\partial \dot{T}_{h}^{(2)}\right|_{t=t_{h}}=0
$$

are automatically satisfied. Therefore, using Eqs. (B6) and (B7), optimum temperatures of the working fluid for exhausting heat to the high temperature thermal reservoir 
are given by

$$
T_{1}^{(2)}=\frac{T_{H}}{\left(1+\frac{\left(1-\xi_{1}\right) \Delta S}{k_{h} t_{1}}\right)},
$$

and

$$
T_{h}^{(2)}=\frac{T_{H}}{\left(1-\frac{\xi_{h} \Delta S}{\mathcal{B} t_{h}}\right)},
$$

where $\xi_{1}$ and $\xi_{h}$ are still undetermined.

The task now is to determine $\xi_{1}^{*}$ and $\xi_{h}^{*}$. Setting

$$
\begin{aligned}
& T_{1}^{(1)}=T_{1}^{(2)}, \\
& \xi_{1}^{*}=\frac{(1-\theta)+\left(\frac{\Delta S}{k_{h} t_{1}}\right)}{\frac{\Delta S}{t_{1}}\left(\frac{\theta}{\alpha}+\frac{1}{k_{h}}\right)},
\end{aligned}
$$

and setting $T_{h}^{(1)}=T_{h}^{(2)}$,

$$
\xi_{h}^{*}=\frac{(1-\theta)+\left(\frac{\Delta S \theta}{k_{1} t_{h}}\right)}{\frac{\Delta S}{t_{h}}\left(\frac{1}{B}+\frac{\theta}{k_{1}}\right)} .
$$


For an FTCRC with non-ideal heat switches that maximizes efficiency, the parameters $\xi_{1}^{*}$ and $\xi_{h}^{*}$ define the optimum fractional amounts of entropy generated in the working fluid during low and high temperature heat transfer, respectively. Using $\xi_{l}^{*}$ and $\xi_{h}^{*}$ with Eqs. $(4.5)-(4.7),(B 18),(B 19)$, (B30) and (B31), the following expressions are obtained for heat transfer:

1) heat transfer from the thermal reservoir at temperature $T_{L}$ into the working fluid at temperature $T_{l}$ is given by

$$
Q_{L 1}\left(\tilde{t}_{1}\right)=\frac{\Delta S T_{2} \Gamma \tilde{E}_{1}\left(1-\Gamma \tilde{E}_{1} \tilde{k}_{1}(\theta-1)\right)}{\left(\Gamma \tilde{E}_{1}\left(1+\tilde{k}_{1}\right)+1\right)},
$$

where the dimensionless variables $\Gamma=\sqrt{\alpha / B}, \tilde{t}_{1}=t_{1} \sqrt{\alpha \beta} / \Delta S$, $\theta=T_{H} / T_{L}$, and $\tilde{k}_{1}=k_{1} / \alpha$ have been used;

2) Heat transfer from the thermal reservoir at temperature $T_{H}$ into the working fluid at temperature $T_{1}$ is given by

$$
Q_{H 1}\left(\tilde{t}_{1}\right)=\frac{\Delta S T_{L} \Gamma \tilde{k}_{1} \tilde{E}_{1}\left(\theta+\Gamma \tilde{E}_{1}(\theta-1)\right)}{\left(\Gamma \tilde{t}_{1}\left(1+\tilde{k}_{1}\right)+1\right)} ;
$$

3) Heat transfer from the working fluid at temperature $T_{h}$ into the thermal reservoir at temperature $T_{H}$ is given by

$$
Q_{h H}\left(\tilde{t}_{h}\right)=\frac{-\Delta S T_{H} \tilde{E}_{h}\left(\Gamma-\tilde{t}_{h} \tilde{k}_{h}(1-1 / \theta)\right)}{\Gamma\left(\tilde{t}_{h}\left(1+\tilde{k}_{h}\right)-\Gamma\right)}
$$


where the dimensionless variables $\tilde{t}_{h}=t_{h} \sqrt{\alpha B} / \Delta S$ and $\tilde{k}_{h}=k_{h} / B$

4) Heat transfer from the working fluid at temperature $T_{h}$ into the thermal reservoir at temperature $T_{L}$ is given by

$$
Q_{h x}\left(\tilde{t}_{h}\right)=\frac{-\Delta S T_{h} \tilde{k}_{h} \tilde{E}_{h}\left(\Gamma+\tilde{E}_{h} \tilde{k}_{h}(\theta-1)\right)}{\Gamma\left(\tilde{t}_{h}\left(1+\tilde{k}_{h}\right)-\Gamma\right)}
$$

Equations (B34) - (B37) agree with Eqs. (4.19), (4.20), (4.28), and (4.29). Therefore, the approach used in chapter IV. for obtaining the optimum heat transfer solutions by extremizing $Q_{h}\left(\tilde{t}_{h}, \tilde{E}_{1}\right)$ and $Q_{1}\left(\tilde{t}_{h}, \tilde{E}_{1}\right)$ is justified because optimum heat transfer solutions are identical to those obtained by extremizing $Q_{H}\left(\tilde{t}_{h}, \tilde{E}_{1}\right)$ and $Q_{L}\left(\tilde{t}_{h}, \tilde{E}_{1}\right)$. 